\title{
Boundary Smoothing Properties of the Korteweg-de Vries Equation in a Quarter Plane and Applications
}

Jerry L. Bona, S. M. Sun, and Bing-Yu Zhang

Communicated by Jerry Bona, received September 14, 2005.

1991 Mathematics Subject Classification. Primary 35; Secondary 76.

Key words and phrases. KdV, boundary smoothing, well-posedness.

JLB was partially supported by the National Science Foundation and by the W. M. Keck Foundation. SMS was partially supported by the National Science Foundation. BYZ was partially supported by the Taft Memorial Fund. 
Abstract. Reported here are results concerning the initial boundary value problem (IBVP) for the Korteweg-de Vries equation in a quarter plane, viz.

$$
\left\{\begin{array}{l}
u_{t}+u_{x}+u u+u_{x x x}=0, \quad \text { for } x, t \geq 0, \\
u(x, 0)=\phi(x), \quad u(0, t)=h(t) .
\end{array}\right.
$$

The present study commences with a representation of solutions of (0.1) derived in our earlier paper [Trans. American Math. Soc. 354 (2001), 427-490]. The problem (0.1) arises naturally in the modeling of various types of wave phenomena, but the focus here will be on two mathematical points, namely a type of boundary smoothing and its impact upon the well-posedness of $(0.1)$ in the $L^{2}$-based Sobolev spaces $H^{s}\left(R^{+}\right)$.

It has been known for some time that the $\mathrm{KdV}$ equation posed on the quarter plane possesses the Kato smoothing property just as do solutions on the whole plane of the pure initial value problem; that is to say

$\phi \in H^{s}\left(R^{+}\right)$and $h \in H_{\text {loc }}^{\frac{s+1}{3}}\left(R^{+}\right)$implies $u \in L^{2}\left(0, T ; H_{l o c}^{s+1}\left(R^{+}\right)\right)$for any finite value of $T$ for which the solution exists on $[0, T]$.

It is shown here that the linear IBVP obtained by dropping the nonlinear term $u u_{x}$ in (0.1) has the following somewhat startling smoothing property:

if $\phi=0$ and $h \in H_{l o c}^{\frac{s+1}{3}}\left(R^{+}\right)$, then the solution $u$ of the linear version of (0.1) belongs to the space $L^{2}\left(0, T ; H^{s+\frac{3}{2}}\left(R^{+}\right)\right)$.

The linearized version of $(0.1)$ with zero initial data, $\phi=0$, has another interesting property. The solution $u(x, t)$ is the restriction to $R^{+} \times R^{+}$of a function $w(x, t)$ defined on $R \times R$ which is such that

$$
\left(\int_{-\infty}^{\infty} \int_{-\infty}^{\infty}(1+|\xi|)^{2 s}\left(1+\left|\tau-\xi^{3}\right|\right)^{2 b}|\hat{w}(\xi, \tau)|^{2} d \xi d \tau\right)^{1 / 2} \leq C\|h\|_{H} \frac{3 b+s-1 / 2}{3}_{\left(R^{+}\right)}
$$

where $b$ is any value in $\left[0, \frac{1}{2}-\frac{s}{3}\right)$ if $-\frac{3}{2} \leq s<\frac{3}{2}, b$ is any value in $\left[0, \frac{5}{6}-\frac{s}{3}\right]$ if $-\frac{1}{2}<s<1$ and $C$ is a constant depending only on $s$ and $b$.

Aided by these boundary integral estimates, and after introduction of suitable versions of the Bourgain spaces whose underlying spatial-temporal domain is a quarter plane, we demonstrate that the full nonlinear IBVP $(0.1)$ is unconditionally locally well-posed in the space $H^{s}\left(R^{+}\right)$for any $s>-\frac{3}{4}$. More precisely, it is shown that

for a given compatible pair $(\phi, h) \in H^{s}\left(R^{+}\right) \times H_{l o c}^{\frac{s+1}{3}}\left(R^{+}\right)$, there exists a $T^{*}>0$ such that the IBVP (0.1) admits a unique mild solution $u \in$ $C\left(\left[0, T^{*}\right], H^{s}\left(R^{+}\right)\right)$, which depends continuously on the initial value $\phi$ and the boundary value $h$.

Moreover, the IBVP $(0.1)$ is shown to be unconditionally globally wellposed in $H^{s}\left(R^{+}\right) \times H_{l o c}^{\frac{s+1}{3}}\left(R^{+}\right)$for $s \geq 3$, while unconditional global wellposedness is shown to hold for $0 \leq s<3$ in $H^{s}\left(R^{+}\right) \times H_{l o c}^{\frac{1+s+\epsilon}{3}}\left(R^{+}\right)$for any $\epsilon>0$.

\section{Contents}

1. Introduction

2. Linear Problems and Extension Formulas 12

3. Boundary smoothing properties 22

4. Well-posedness 42

5. Appendices $\quad 53$

$\begin{array}{ll}\text { References } & 67\end{array}$ 


\section{Introduction}

In this paper, we continue the study of the initial-boundary-value problem for the Korteweg-de Vries (KdV) equation posed in a quarter plane, namely

$$
\left.\begin{array}{l}
u_{t}+u_{x}+u u_{x}+u_{x x x}=0, \quad \text { for } x, t \geq 0, \\
u(x, 0)=\phi(x), \quad u(0, t)=h(t) .
\end{array}\right\}
$$

As pointed out by several authors, (see [1] for an early commentary in the context of the BBM-equation), initial-boundary-value problems of the form (1.1) may serve as models for waves generated by a wave maker in a channel, or for waves approaching shallow water (e.g. the shore) from deep water. Similar problems arise in other physical contexts where KdV-type equations serve as models. Here, two mathematical issues connected to (1.1) will be addressed; boundary smoothing properties and the well-posedness of this initial-boundary value problem (IBVP henceforth) in the $L^{2}$-based Sobolev spaces $H^{s}\left(R^{+}\right)$. The overall thrust of our theory is that stronger boundary smoothing properties than heretofore noticed allow the formulation of a sharper well-posedness theory.

We begin with a review of existing theory which provides a setting in which to state precisely our results and put them into present day context. Recall that for the pure initial-value problem (IVP henceforth) for the KdV-equation

$$
u_{t}+u u_{x}+u_{x x x}=0, \quad u(x, 0)=\phi(x), \quad x, t \in R,
$$

written in traveling coordinates, it is well-known that there is no gain or loss of regularity in the Sobolev classes $H^{s}(R)$. As Saut and Temam [38] pointed out, for any $t \in R$,

$$
u(\cdot, t) \in H^{s}(R) \quad \text { if and only if } \quad \phi \in H^{s}(R),
$$

at least for suitable values of $s$. There is, however, more subtle smoothing associated with the initial-value problem (1.2). In the late 1970's, Kato [28, 29] discovered that for solutions of (1.2),

$$
\phi \in H^{s}(R) \text { implies that } u \in L^{2}\left(0, T ; H_{l o c}^{s+1}(R)\right) .
$$

This property, now known as Kato-smoothing, stimulated an extensive investigation of various smoothing properties associated with solving the KdV-equation and other dispersive wave equations (see, for example, $[10,11, \mathbf{1 4}, \mathbf{3 1}, \mathbf{3 2}, \mathbf{3 3}, \mathbf{3 4}, \mathbf{3 9}, \mathbf{4 3}]$ and the references contained therein). In particular, Kenig, Ponce and Vega [31] demonstrated that, when $\phi \in H^{s}(R)$ with $s>\frac{3}{4}$, there is a unique solution $u$ of (1.2) which belongs to the space $C\left(R ; H^{s}(R)\right)$ and is such that

$$
\begin{gathered}
\left(\sup _{-\infty<x<+\infty} \int_{-T}^{T}\left|\partial_{x}^{s+1} u(x, t)\right|^{2} d t\right)^{\frac{1}{2}} \leq C\|\phi\|_{H^{s}(R)}, \\
\left(\int_{-T-\infty<x<+\infty}^{T} \sup _{x}\left|\partial_{x} u(\cdot, t)\right|^{4} d t\right)^{\frac{1}{4}} \leq C\|\phi\|_{H^{s}(R)}
\end{gathered}
$$

and

$$
\left(\int_{-\infty}^{+\infty} \sup _{-T \leq t \leq T}|u(x, t)|^{2} d x\right)^{\frac{1}{2}} \leq C(1+T)\|\phi\|_{H^{s}(R)}
$$


where the constants $C$ on the right-hand sides depend only on $s$ and on $T$ when it appears. The inequality (1.4) is a sharp version of Kato smoothing and (1.5) is sometimes called global smoothing of Strichartz type, while (1.6) reveals a kind of global temporal smoothing.

In the early 1990's, in attempting to establish the well-posedness of (1.2) in $H^{s}(R)$ for smaller values of $s$, Bourgain [11] found a yet more subtle smoothing property for solutions of (1.2). This property may be expressed as follows: for $\phi \in H^{s}(R)$ with $s \geq 0,(1.2)$ admits a solution $u \in C\left([0, T] ; H^{s}(R)\right)$ which is the restriction to $R \times(0, T)$ of a function $w$ on the whole plane $R \times R$ such that

$$
\left(\int_{-\infty}^{\infty} \int_{-\infty}^{\infty}(1+|\xi|)^{2 s}\left(1+\left|\tau-\xi^{3}\right|\right)|\hat{w}(\xi, \tau)|^{2} d \xi d \tau\right)^{1 / 2} \leq C\|\phi\|_{H^{s}(R)}
$$

where $C$ depends only on $s$ and $\hat{w}$ is the Fourier transform of $w$ with respect to both of the independent variables. Because of this smoothing property of solutions, Bourgain could show that (1.2) is (conditionally) well-posed in $H^{s}(R)$ for $s \geq$ 0 . (The distinction between well-posedness and conditional well-posedness will be drawn presently.) Later, the smoothing property (1.7) was improved by Kenig, Ponce and Vega $[\mathbf{3 3}, \mathbf{3 4}]$ to the stronger property

$$
N_{s, b}(w) \equiv\left(\int_{-\infty}^{\infty} \int_{-\infty}^{\infty}(1+|\xi|)^{2 s}\left(1+\left|\tau-\xi^{3}\right|\right)^{2 b}|\hat{w}(\xi, \tau)|^{2} d \xi d \tau\right)^{1 / 2} \leq C\|\phi\|_{H^{s}(R)}
$$

for any $\phi \in H^{s}(R)$ with $s>-\frac{3}{4}$, where $\frac{1}{2}<b<1$ depends only on $s$ and $C$ depends on $s$ and $b$. Note that if $s \in R$ and $\mathcal{U}$ is the unitary group in $H^{s}(R)$ defined by

$$
\mathcal{U}(t)=\exp \left(i t P\left(D_{x}\right)\right)
$$

where $P\left(D_{x}\right)$ is the Fourier multiplier with symbol $P(\xi)=\xi^{3}$, then (cf. [36])

$$
N_{s, b}(w) \sim\|\mathcal{U}(-t) w\|_{H^{s, b}}
$$

where $H^{s, b} \equiv H_{t}^{b}\left(R ; H_{x}^{s}(R)\right)$. For given $f \in H^{s, b}$, the larger the value of $b$, the smoother is $f$ with respect to time $t$. In particular, if $b>\frac{1}{2}, H^{s, b}$ is continuously embedded into the space $C\left(R ; H_{x}^{s}(R)\right)$. The inequality (1.8) allowed Kenig, Ponce and Vega to show that, locally in time, the IVP (1.2) is (conditionally) well-posed in $H^{s}(R)$ provided only that $s>-\frac{3}{4}$. This result was recently strengthened to include (conditional) global well-posedness in the same function classes, by Colliander, et al. in $[\mathbf{1 3}]$.

For the IBVP (1.1), the Kato smoothing property for (1.1) was established by Bona and Winther $[\mathbf{8 , 9}]$, where they showed that solutions lie in $L_{2}\left(0, T ; H_{l o c}^{n+1}\left(R^{+}\right)\right)$ if $\phi \in H^{n}\left(R^{+}\right)$and $h \in H_{l o c}^{n+2}\left(R^{+}\right)$for $n \geq 2$. Smoothing properties analogous to (1.4)-(1.6) were established by Bona, Sun and Zhang. These were derived in [4] in the following form.

For $s>\frac{3}{4}$, if $\phi \in H^{s}\left(R^{+}\right)$and $h \in H_{l o c}^{\frac{s+1}{3}}\left(R^{+}\right)$satisfy certain compatibility conditions at $(x, t)=(0,0)$, then the $\operatorname{IBVP}(1.1)$ admits a unique solution

$$
u \in C\left(0, T ; H^{s}\left(R^{+}\right)\right) \cap L^{2}\left(0, T ; H_{l o c}^{s+1}\left(R^{+}\right)\right),
$$

which satisfies the additional properties

$$
\left(\sup _{0<x<+\infty} \int_{0}^{T}\left|\partial_{x}^{s+1} u(x, t)\right|^{2} d t\right)^{\frac{1}{2}} \leq C\left(\|\phi\|_{H^{s}\left(R^{+}\right)}+\|h\|_{H^{\frac{s+1}{3}(0, T)}}\right),
$$




$$
\left(\int_{0}^{T} \sup _{0<x<+\infty}\left|\partial_{x} u(\cdot, t)\right|^{4} d t\right)^{\frac{1}{4}} \leq C\left(\|\phi\|_{H^{s}(R)}+\|h\|_{H^{\frac{s+1}{3}(0, T)}}\right)
$$

and

$$
\left(\int_{0}^{+\infty} \sup _{0 \leq t \leq T}|u(x, t)|^{2} d x\right)^{\frac{1}{2}} \leq C\left(\|\phi\|_{H^{s}(R)}+\|h\|_{H^{\frac{s+1}{3}(0, T)}}\right)
$$

where the constants depend only on $s$ and $T$. As just stated, the results in [4] were local in time; corresponding global results were also established but were only optimal if $s \geq 3$. Global results very nearly corresponding to the local theory in [4] are obtained in the recent paper [19] of Faminskii.

Recently, Colliander and Kenig [12] in a paper concerned with the IBVP for the generalized $\mathrm{KdV}$-equation wherein $u u_{x}$ is replaced by $u^{p} u_{x}$, showed in the case $p=1$ that solutions of (1.1) also possess a Bourgain smoothing property which can be expressed precisely as follows. For $\phi \in L^{2}\left(R^{+}\right)$and $h \in H^{\frac{1}{3}}\left(R^{+}\right)$, (1.1) admits a solution $u \in C\left(0, T ; L^{2}\left(R^{+}\right)\right)$which is the restriction of a function $w(x, t)$ defined on the whole plane satisfying

$$
\tilde{\Lambda}_{0, b}^{\alpha}(w) \leq C\left(\|\phi\|_{H^{s}(R)}+\|h\|_{H^{\frac{1}{3}\left(R^{+}\right)}}\right)
$$

where

$$
\tilde{\Lambda}_{s, b}^{\alpha}(w)=\left(N_{s, b}^{2}(w)+\int_{-\infty}^{\infty} \int_{-1}^{1}(1+|\tau|)^{2 \alpha}|\hat{w}(\xi, \tau)|^{2} d \xi d \tau\right)^{\frac{1}{2}}
$$

with $\alpha>\frac{1}{2}$ and where the constant $b$ is required to be strictly less than $\frac{1}{2}$ in contrast to the theory for the IVP (1.2), where $b>\frac{1}{2}$ obtains.

The discussion is now turned more directly to the contributions in the present essay. We commence with boundary smoothing properties. Note first that if the boundary value $h$ in (1.1) vanishes identically, the solution $u$ satisfies the energy identity

$$
\frac{d}{d t} \int_{0}^{\infty} u^{2}(x, t) d x+u_{x}^{2}(0, t)=0 \quad \text { for all } t \geq 0 .
$$

Thus, the $L^{2}$-norm of the solution $u$ is decreasing and is strictly so as long as $u_{x}(0, t) \neq 0$. This suggests that some dissipative mechanism is introduced through imposition of the boundary condition at $x=0$. An interesting question arises naturally in this situation:

\section{Can one quantify this boundary dissipative effect?}

It is well-known that a solution often becomes smoother under the influence of dissipative effects. Thus a further question presents itself: effect?

Do solutions of (1.1) becomes smoother because of this boundary dissipative

To address these issues, it is helpful to consider carefully the linear problem

$$
\left.\begin{array}{ll}
u_{t}+u_{x}+u_{x x x}=0, & x, t \in R^{+}, \\
u(x, 0)=0, \quad u(0, t)=h(t), & x, t \in R^{+}
\end{array}\right\}
$$


associated to (1.1) and present some new boundary smoothing properties for its solutions. The Kato-smoothing result of [8], when extended to fractional-order spaces, states that the solution $u$ of (1.13) belongs to the space

$$
C\left(R^{+} ; H^{s}\left(R^{+}\right)\right) \cap L^{2}\left(R^{+} ; H_{l o c}^{s+1}\left(R^{+}\right)\right)
$$

if $h \in H_{0}^{\frac{s+1}{3}}\left(R^{+}\right)$, at least if $s$ is not too small. The space $H_{0}^{r}\left(R^{+}\right)$is the closure of $\mathcal{D}\left(R^{+}\right)$in $H^{r}\left(R^{+}\right)$, as usual. It will be demonstrated in this paper that (1.13) possesses the following additional boundary smoothing properties.

THEOREM 1.1. For a given pair $(b, s)$ satisfying

$$
\left\{\begin{array}{l}
0 \leq b<\frac{1}{2}-\frac{s}{3} \quad \text { if } s \leq 0, \text { or } \\
0 \leq b<\frac{5}{6}-\frac{s}{3} \quad \text { if }-\frac{1}{2}<s<1
\end{array}\right.
$$

there exists a constant $C$ depending only on $s$ and $b$ such that for any $h \in H_{0}^{\frac{s+1}{3}}\left(R^{+}\right)$, the corresponding solution $u$ of (1.13) is the restriction of a function $w(x, t)$ defined on the whole plane satisfying

$$
N_{s, b}(w) \leq C\|h\|_{H} \frac{3 b+s-1 / 2}{3}\left(R^{+}\right) .
$$

Remark: Notice that this improves upon the just described result of Colliander and Kenig [12] both as regards the range of $b$ (solutions are seen to be smoother in $t$ ) and by allowing for negative values of $s$.

As a corollary, there appears the following boundary smoothing property for solutions of the IBVP (1.13).

THEOREM 1.2. Let $s \geq-\frac{3}{2}$ and $T>0$ be given. There exists a constant $C$ such that for any $h \in H_{0}^{\frac{s+1}{3}}\left(R^{+}\right)$, the corresponding solution $u$ of (1.13) belongs to the space $L^{2}\left(0, T ; H_{0}^{s+\frac{3}{2}}\left(R^{+}\right)\right)$and satisfies

$$
\|u\|_{L^{2}\left(0, T ; H^{s+\frac{3}{2}}\left(R^{+}\right)\right)} \leq C\|h\|_{H^{\frac{s+1}{3}}\left(R^{+}\right)}
$$

for a constant $C$ depending only on $s$ and $T$.

\section{Remarks:}

(i) The smoothing property presented by (1.16) is global in the spatial variable $x$.

(ii) This smoothing property only holds for (1.13); it is not valid for the linear IBVP associated to (1.1) nor for the nonlinear problem (1.1).

(iii) For any $T>0$ and $\epsilon>0$, the following estimates were established by Faminskii [18] for the solution $u$ of (1.13);

$$
\begin{gathered}
\|u\|_{C\left(0, T ; L^{2}\left(R^{+}\right)\right)} \leq C(T, \epsilon)\|h\|_{L^{6+\epsilon}(0, T)}, \\
\left\|u_{x}(x, \cdot)\right\|_{L_{t}^{1}\left(0, T ; L_{x}^{\infty}\left(R^{+}\right)\right)} \leq C\|h\|_{H^{\frac{1}{3}+\epsilon}\left(R^{+}\right)}
\end{gathered}
$$

and

$$
\left\|u_{x}\right\|_{L_{2}\left(R^{+} \times R^{+}\right)} \leq C\|h\|_{H^{\frac{1}{6}\left(R^{+}\right)}} .
$$


(iv) As a direct consequence of estimate (1.16), we have

$$
\left\|u_{x}(x, \cdot)\right\|_{L_{t}^{2}\left(0, T ; L_{x}^{\infty}\left(R^{+}\right)\right)} \leq C\|h\|_{H^{\frac{1}{3}+\epsilon}\left(R^{+}\right)}
$$

which is slightly stronger than (1.18).

(v) The estimate (1.18) plays a key role in establishing sharper global wellposedness results for (1.1) in [19].

In addition, two improved versions of Bourgain smoothing are developed here for the nonlinear IBVP (1.1). These take the following form.

THEOREM 1.3. For given $s$ in the interval $-\frac{3}{4}<s \leq 1$, there exists a constant $b \in\left(0, \frac{1}{2}\right]$ depending on $s$ such that for $\phi \in H^{s}\left(R^{+}\right)$and $h \in H^{\frac{3 b+s-1 / 2}{3}}\left(R^{+}\right)$, with $\phi(0)=h(0)$ in case $s>\frac{1}{2}$, the $\operatorname{IBVP}(1.1)$ admits a solution $u \in C\left(0, T ; H^{s}\left(R^{+}\right)\right)$ which is the restriction to the domain $R^{+} \times(0, T)$ of a function $w(x, t)$, defined on the whole plane, satisfying

$$
\tilde{\Lambda}_{s, b}^{\alpha}(w) \leq C\left(\|\phi\|_{H^{s}(R)}+\|h\|_{H} \frac{3 b+s-1 / 2}{3}\left(R^{+}\right)\right)
$$

for some $\alpha>\frac{1}{2}$. In particular, if $h \in H^{\frac{s+1}{3}}\left(R^{+}\right)$, then

$$
\tilde{\Lambda}_{s, \frac{1}{2}}^{\alpha}(w) \leq C\left(\|\phi\|_{H^{s}(R)}+\|h\|_{H^{\frac{s+1}{3}}\left(R^{+}\right)}\right) \text {. }
$$

\section{Remarks:}

(i) In case $s=0,(1.22)$ is a slightly stronger version of the estimate (1.12) due to Colliander and Kenig $[\mathbf{1 2}]$ in that it allows $b=\frac{1}{2}$ instead of asking that $b$ be strictly less than $\frac{1}{2}$.

(ii) As pointed out earlier, one needs that $\phi \in H^{s}\left(R^{+}\right)$and $h \in H_{l o c}^{\frac{s+1}{3}}\left(R^{+}\right)$to have the solution $u$ of the IBVP (1.1) belonging to the space $C\left(0, T ; H^{s}\left(R^{+}\right)\right)$. However, when $b<\frac{1}{2}$,

$$
\frac{3 b+s-1 / 2}{3}<\frac{1+s}{3} .
$$

Estimate (1.21) thus reveals a boundary smoothing property for the nonlinear problem (1.1).

The second main issue addressed in this paper is the well-posedness of the IBVP (1.1). Here and above, well-posedness means existence and uniqueness of solutions, and continuous dependence of solutions on auxiliary data. The following definition encapsulates the precise sense of well posedness enforced here.

Definition 1.4 (well-posedness). Let $s, s^{\prime} \in R$ be given. The IBVP (1.1) is said to be (locally) well-posed in the space $H^{s}\left(R^{+}\right) \times H_{\text {loc }}^{s^{\prime}}\left(R^{+}\right)$if for any $r>0$ there exists a $T=T(r)>0$ with $T(r) \rightarrow \infty$ as $r \rightarrow 0$ such that for given $\phi \in H^{s}\left(R^{+}\right)$ and $h \in H_{\text {loc }}^{s^{\prime}}\left(R^{+}\right)$satisfying suitable compatibility conditions, and if

$$
\|\phi\|_{H^{s}\left(R^{+}\right)}+\|h\|_{H^{s^{\prime}(0, T)}} \leq r,
$$

then (1.1) admits a unique solution $u=u(x, t)$ in the space $C\left(0, T ; H^{s}\left(R^{+}\right)\right)$. Moreover, the solution depends continuously on its initial data $\phi$ and its boundary value $h$ in the corresponding spaces.

\section{Remarks:}


(i) The well-posedness described above is called local well-posedness since the $T$ in the above definition may depend on $r$. If $T$ may be taken to be independent of $r$, then (1.1) is said to be globally well-posed in the space $H^{s}\left(R^{+}\right) \times H_{l o c}^{s^{\prime}}\left(R^{+}\right)$.

(ii) By a standard scaling argument, the above definition of well-posedness is equivalent to the following statement:

There exists a $\delta$ depending only on $s$ and $s^{\prime}$, such that for given $\phi \in$ $H^{s}\left(R^{+}\right)$and $h \in H_{\text {loc }}^{s^{\prime}}\left(R^{+}\right)$satisfying suitable compatibility conditions and having

$$
\|\phi\|_{H^{s}\left(R^{+}\right)}+\|h\|_{H^{s^{\prime}(0,1)}} \leq \delta
$$

then (1.1) admits a unique solution $u=u(x, t)$ in the space $C\left(0,1 ; H^{s}\left(R^{+}\right)\right)$. Moreover, the solution depends continuously on its initial data $\phi$ and its boundary value $h$ in the corresponding spaces.

(iii) There is a weaker notion discussed by Kato [30] of conditional wellposedness in which solutions are only known to be unique if they satisfy additional auxiliary conditions. Solutions satisfying such conditions are often available via the contraction mapping principle applied to an associated integral equation, but they are not necessarily known to be unique in the broader class not respecting the extra conditions. This point will be further elaborated presently.

The mathematical study of the IBVP (1.1) began with the work of Ton [42] in which, existence and uniqueness were established assuming that the initial data $\phi$ is smooth and the boundary data $h \equiv 0$. The first well-posedness result in the strict sense of Definition 1.4 for the IBVP (1.1) was presented by Bona and Winther $[8,9]$.

Theorem A The IBVP (1.1) is (globally) well-posed in the space $H^{3 k+1}\left(R^{+}\right) \times$ $H_{l o c}^{k+1}\left(R^{+}\right)$for $k=1,2, \cdots$.

Faminskii, in a wide-ranging paper [16], deals with the IBVP (1.1) for a generalization of the KdV-equation somewhat like that appearing later in Craig, Kappeler and Strauss [15]. He puts forward a theory of well-posedness for generalized solutions set in weighted $H^{1}$-Sobolev classes. Moreover, he obtains extra interior regularity in case the initial data decays suitably rapidly at $+\infty$. In [4], Bona, Sun and Zhang obtained the following conditional well-posedness result for (1.1).

Theorem B The IBVP (1.1) is locally well-posed in the space $H^{s}\left(R^{+}\right) \times H_{l o c}^{\frac{s+1}{3}}\left(R^{+}\right)$ for $s>3 / 4$ with the following auxiliary condition to ensure uniqueness;

$$
\text { the solution } u \text { satisfies the estimates (1.9), (1.10) and (1.11). }
$$

Remarks: Notice that the last result reveals the relationship

$$
s^{\prime}=\frac{s+1}{3}
$$

in the notation of the definition of well-posedness. This turns out to be the natural consequence of the balance

$$
\partial_{t} \sim \partial_{x}^{3}
$$

It was not noticed in the early attacks [8,9,16-19,42] on (1.1). 
In Theorem A, solutions are in fact classical, which is to say all the terms in the equation are bounded and continuous functions of $(x, t)$ and the equation is satisfied identically. In Theorem B, the solutions are distributional, but of course have the further regularity attached to lying in $C\left(0, T ; H^{s}\left(R^{+}\right)\right)$and satisfying (1.9), (1.10) and (1.11).

The following result for (1.1) was established recently by Colliander and Kenig $[12]$.

Theorem C ${ }^{1}$ For any $\phi \in H^{s}\left(R^{+}\right)$and $h \in H^{\frac{s+1}{3}}\left(R^{+}\right)$with $0 \leq s \leq 1$ which satisfy the compatibility condition $\phi(0)=h(0)$ if $s>\frac{1}{2}$, there exists a $T=T(\phi, h)>$ 0 and a solution $u \in C\left(0, T ; H^{s}\left(R^{+}\right)\right)$of the $\operatorname{IBVP}(1.1)$. The map $(\phi, h) \rightarrow u$ is Lipschitz-continuous from $H^{s}\left(R^{+}\right) \times H^{\frac{s+1}{3}}\left(R^{+}\right)$to $C\left(0, T ; H^{s}\left(R^{+}\right)\right)$.

This is not a well-posedness result in the sense of Definition 1.4, since uniqueness is not discussed. Actually, a well-posedness result is established for an integral equation

$$
w=H S_{1}(\phi, h)+I H S_{1}\left(w w_{x}\right)
$$

posed on the whole plane $R \times R$, where $H S_{1}(\phi, h)$ is an integral operator associated to the linear homogeneous problem

$$
\left.\begin{array}{l}
v_{t}+v_{x}+v_{x x x}=0, \quad x>0, t \in(0, T), \\
w(x, 0)=\phi(x), \quad w(0, t)=h(t), \quad x>0, t \in(0, T)
\end{array}\right\}
$$

and $I H S_{1}(f)$ is an integral operator associated with the linear inhomogeneous problem

$$
\left.\begin{array}{l}
v_{t}+v_{x}+v_{x x x}=f, \quad x>0, t \in(0, T), \\
w(x, 0)=0, \quad w(0, t)=0, \quad x>0, t \in(0, T) .
\end{array}\right\}
$$

The precise definitions of the integral operators $H S_{1}$ and $I H S_{1}$ are given in [12]. The relation between (1.24) and the IBVP (1.1) (without the linear transport term $u_{x}$ in the equation) is that a solution $w$ of $(1.24)$ on $R \times R$, when restricted to the domain $R^{+} \times(0, T)$, is a solution of (1.1). For the integral equation (1.24), Colliander and Kenig established the following well-posedness result.

Theorem $\mathbf{D}$ Let $0 \leq s \leq 1$ be given with $s \neq \frac{1}{2}$. There exists a $\delta>0$ such that, if $(\phi, h) \in H^{s}\left(R^{+}\right) \times H^{\frac{s+1}{3}}\left(R^{+}\right)$satisfies

$$
\|(\phi, h)\|_{H^{s}\left(R^{+}\right) \times H^{\frac{s+1}{3}}\left(R^{+}\right)} \leq \delta
$$

and $\phi(0)=h(0)$ when $s>\frac{1}{2}$, then the integral equation (1.24) admits a unique solution $u \in C\left(R ; L_{x}^{2}(R)\right)$ satisfying the auxiliary condition

$$
\tilde{\Lambda}_{s, b}^{\alpha}(w)<\infty
$$

for some $\alpha>\frac{1}{2}$ and $b$ in the range $0<b<\frac{1}{2}$ (see the text following (1.12) above).

The well-posedness of (1.1) presented in Theorem B and Theorem D is conditional rather than in the sense of Definition 1.4 since auxiliary conditions are needed to ensure the uniqueness. By contrast, the well-posedness of (1.1) presented in Theorem A is in the strict sense of Definition 1.4 and is unconditional.

\footnotetext{
${ }^{1}$ This result has been extended recently by J. Holmer [25] to the case $-\frac{3}{4}<s<0$.
} 
The issue of conditional well-posedness also appears in the works of Bourgain, and Kenig, Ponce and Vega for the IVP (1.2) where the uniqueness is established only for solutions in the space $C\left(-T, T ; H^{s}(R)\right)$ satisfying certain auxiliary conditions. A basic question is are these auxiliary conditions really essential to the uniqueness? The reader is referred to $[\mathbf{7}, \mathbf{3 0}]$ for further discussion of unconditional and conditional well-posedness for general classes of nonlinear evolution equations.

The issue is more interesting than might appear at first glance. There are many ways to transform the IBVP (1.1) into an integral equation. Most of these admit an analysis something like that made in [12] leading to Theorem $\mathrm{C}$. The question is, when two such solutions are restricted to $R^{+} \times(0, T)$ for some $T>0$, are they equal to each other? For the linear problem, this is established in $[\mathbf{1 2}]$, but the point is unresolved for the nonlinear problem.

One of the main theorems proved in this paper is the following well-posedness result for (1.1), which also resolves the uniqueness issue for the nonlinear problem just mentioned.

TheOrem 1.9. Let $s \in\left(-\frac{3}{4}, 1\right]$ and $T>0$ be given. For any $\phi \in H^{s}\left(R^{+}\right)$and $h \in H^{\frac{s+1}{3}}(0, T)$ satisfying the compatibility condition

$$
\phi(0)=h(0)
$$

when $s>\frac{1}{2}$, there exists a $T^{*}>0$ depending only on $\|(\phi, h)\|_{H^{s}\left(R^{+}\right) \times H^{\frac{s+1}{3}}(0, T)}$ such that the $\operatorname{IBVP}(1.1)$ admits a unique solution $u \in C\left(0, T^{*} ; H^{s}\left(R^{+}\right)\right)$which is the restriction to $R^{+} \times[0, T]$ of a function $w=w(x, t)$ satisfying the estimate

$$
\tilde{\Lambda}_{s, \frac{1}{2}}^{\alpha}(w)<\infty
$$

for some $\alpha>\frac{1}{2}$.

\section{Remarks:}

(i) The solution given by this Theorem is smoother than that given by Theorem $D$ of Colliander and Kenig, and by Holmer since $b=\frac{1}{2}$ rather than being strictly less than $\frac{1}{2}$.

(ii) The theorem still holds if we replace $\frac{1}{2}$ by some $b<\frac{1}{2}$ in (1.25). Thus the solutions given by Colliander and Kenig in Theorem $D$ and the solution provided by Theorem 1.9 are the same when restricted to $R^{+}$.

Theorem 1.9 is also a conditional well-posedness result. It is natural to speculate whether or not the auxiliary condition (1.25) is removable. A way of resolving this issue is to introduce a concept of mild solution for the IBVP (1.1).

Definition 1.10 (mild solution). Let $s<3$ and $T>0$ be given. For given $\phi \in H^{s}\left(R^{+}\right)$and $h \in H_{l o c}^{\frac{s+1}{3}}\left(R^{+}\right)$, a function $u \in C\left(0, T ; H^{s}\left(R^{+}\right)\right)$is said to be a mild solution of (1.1) on the time interval $[0, T]$ if there exists a sequence $\left\{u_{n}\right\}_{n=1}^{\infty}$ in the space

$$
C\left(0, T ; H^{3}\left(R^{+}\right)\right) \cap C^{1}\left(0, T ; L^{2}\left(R^{+}\right)\right)
$$

with

$$
\phi_{n}(x)=u_{n}(x, 0), \quad h_{n}(t)=u_{n}(0, t), \quad n=1,2, \cdots,
$$

such that 
(i) $u_{n}$ solves the equation in (1.1) in $L^{2}\left(R^{+}\right)$for $0<t<T$, which is to say, each term in the equation lies in $C\left(0, T ; L^{2}\left(R^{+}\right)\right)$and the equation is satisfies for each $t$, almost everywhere in space;

(ii) $\lim _{n \rightarrow \infty} \sup _{0<t<T}\left\|u_{n}(\cdot, t)-u(\cdot, t)\right\|_{H^{s}\left(R^{+}\right)}=0$;

(iii) $\lim _{n \rightarrow \infty}\left\|\phi_{n}-\phi\right\|_{H^{s}\left(R^{+}\right)}=0$ and $\lim _{n \rightarrow \infty}\left\|h_{n}-h\right\|_{H^{\frac{s+1}{3}}(0, T)}=0$.

Remark: A mild solution is a weak solution when $s \geq 0$, but not necessarily vice versa. However, a mild solution might not on the face of it be a distributional solution when $s<0$ since $u^{2}$ may not be a well-defined distribution. Classical energy arguments demonstrate uniqueness of solutions if $s>\frac{3}{2}$. Hence, while larger values of $s$ can be encompassed by demanding the sequence $\left\{u_{n}\right\}_{n=1}^{\infty}$ be drawn from even smoother function classes, there is no need for this in the present context.

We will show that the following facts hold about mild solutions.

THEOREM 1.11 (existence and uniqueness).

(a) The weak solutions given by Theorem B, Theorem $C$ and Theorem 1.9 are all mild solutions.

(b) For given $\phi \in H^{s}\left(R^{+}\right)$and $h \in H_{l o c}^{\frac{s+1}{3}}\left(R^{+}\right)$with $s>-\frac{3}{4}$, the IBVP (1.1) admits at most one mild solution.

An immediate consequence of this theorem is that the auxiliary conditions in Theorem B, Theorem $\mathrm{C}$ and Theorem 1.9 are not essential for the uniqueness and all of them can be removed.

If the appellation solution in Definition 1.4 is understood as mild solution, then we have the following unconditional well-posedness results as one of the main theorems in this paper.

THEOREM 1.12 (unconditional well-posedness). The IBVP (1.1) is unconditionally (locally) well-posed in the space $H^{s}\left(R^{+}\right) \times H_{l o c}^{\frac{s+1}{3}}\left(R^{+}\right)$for $s>-\frac{3}{4}$. Its solution $u$ has the additional properties:

$$
\begin{cases}u \text { satisfies the estimates (1.9)-(1.11) } & \text { if } s>\frac{3}{4} \\ u \text { satisfies the estimates (1.22) } & \text { if }-\frac{3}{4}<s<1 .\end{cases}
$$

Remark: As a model of real wave phenomena, the Korteweg-de Vries equation is not derived to take account of singularity formulation. Thus, one would hope that global well-posedness results obtain for (1.1). Indeed this was shown to be the case in $H^{s}\left(R^{+}\right) \times H_{l o c}^{\frac{s+1}{3}}\left(R^{+}\right)$in $[4]$ for $s \geq 3$. For $1 \leq s \leq 3$, the results of [4] only yielded conditional global well-posedness in the slightly smaller space $H^{s}\left(R^{+}\right) \times H_{l o c}^{\frac{7+3 s}{12}}\left(R^{+}\right)$. Faminskii's recent work [19] showed that (1.1) is conditionally globally well-posed in the space $H^{s}\left(R^{+}\right) \times H_{\text {loc }}^{\frac{1+s+\epsilon}{3}}\left(R^{+}\right)$when $0 \leq s<3$, but leaves open the question of whether the well-posedness is unconditional or not.

Concerning global well-posedness, the following result follows readily from the local theory in Theorem 1.12.

THEOREM 1.13 (global well-posedness). The $\operatorname{IBVP}$ (1.1) is unconditionally globally well-posed in the space $H^{s}\left(R^{+}\right) \times H_{l o c}^{\frac{1+s+\epsilon}{3}}\left(R^{+}\right)$for $0 \leq s<3$ and is unconditionally globally well-posed in the space $H^{s}\left(R^{+}\right) \times H_{l o c}^{\frac{1+s}{3}}\left(R^{+}\right)$for $s \geq 3$. 
The paper is organized as follows. In Section 2, explicit representation formulas are recalled for solutions of initial-boundary-value problems for the linear $\mathrm{KdV}$ equation. These were developed in our earlier paper [4] and will be used to establish the main theorems of this paper. We will demonstrate in this section how to convert the IBVP (1.1) posed in a quarter plane to an integral equation posed on the whole plane, which sets the stage for using powerful tools developed by Bourgain, Kenig, Ponce and Vega and others to study the well-posedness of the IVP. Three different types of extension of the boundary integral operator associated to the non-homogeneous linear boundary value problem (1.13) are provided, which are denoted by $\mathcal{B} I_{e}(t), \mathcal{B} I_{m 1}(t)$ and $\mathcal{B} I_{m 2}(t)$, respectively (see Section 2 for the precise definitions of those operators). Among those operators, $\mathcal{B} I_{e}(t)$ is the simplest; it is basically an even extension (with respect to the spatial variable $x$ ) of the boundary integral operator, from the half line $R^{+}$to the whole line $R$. Using this operator, we are able to prove Theorem 1.1 in case $0 \geq s \geq-\frac{1}{2}$. If $s<-\frac{1}{2}$, one only has the estimate:

$$
N_{s, b}(w) \leq C\|h\|_{H^{\frac{3 b-1}{3}}}\left(R^{+}\right)
$$

instead of the inequality (1.15) featured in Theorem 1.1. Because of this state of affairs, the more complicated boundary operators $\mathcal{B} I_{m 1}(t)$ and $\mathcal{B} I_{m 2}(t)$ are introduced. The operator $\mathcal{B} I_{m 1}(t)$ is helpful when $s<0$, whereas $\mathcal{B} I_{m 2}(t)$ is effective when $0 \leq s<1$.

In Section 3, attention is given to the non-homogeneous linear boundary value problem (1.13). It is demonstrated there that the aforementioned global boundary smoothing properties obtain, as expounded in Theorem 1.1 and Theorem 1.2. The boundary integral operators $\mathcal{B} I_{m 1}(t)$ and $\mathcal{B} I_{m 2}(t)$ play a crucial role in the analysis.

In Section 4, it is shown that the IBVP (1.1) is locally unconditionally wellposed in the space $H^{s}\left(R^{+}\right) \times H_{l o c}^{\frac{s+1}{3}}\left(R^{+}\right)$for $s>-\frac{3}{4}$ and is globally unconditionally well-posed in the same space for $s \geq 3$ and, for any $\epsilon>0$, in the space $H^{s}\left(R^{+}\right) \times$ $H_{l o c}^{\frac{s+1+\epsilon}{3}}\left(R^{+}\right)$when $0 \leq s<3$.

The last section consists of two appendices. The proofs of some technical lemmas used in Section 4 are presented in Appendix I. The results are based on minor modification of arguments already in the literature. The proofs are sketched for the convenience of readers. Some discussion of the boundary integral operator $\mathcal{B} I_{e}$ is provided in Appendix II. The outcome of the analysis is a result showing that estimates involving $\mathcal{B} I_{e}(t)$ alone do not suffice to prove Theorem 1.1 , thereby providing a reason for the introduction of the more complicated boundary operators $\mathcal{B} I_{m 1}$ and $\mathcal{B} I_{m 2}$.

\section{Linear Problems and Extension Formulas}

This section is divided into two subsections. In the first, some explicit representation formulas are recalled from [4] for solutions of initial-boundary-value problems for the linear KdV-equation. Then, a method is put forward to convert the IBVP (1.1) posed on a quarter plane to an integral equation posed on the whole plane. The second subsection features a discussion of the aforementioned three different extensions to $R \times R$ of the boundary integral operator associated to the boundary value problem (2.1) given below. These extended boundary integral operators will play a central role in our analysis. 


\subsection{Explicit solution formulas for linear problems}

First, consider the non-homogeneous problem

$$
\left.\begin{array}{l}
u_{t}+u_{x}+u_{x x x}=0, \quad \text { for } x, t \geq 0 \\
u(x, 0)=0, \quad u(0, t)=h(t) .
\end{array}\right\}
$$

Its solution may be written in the form (see [4])

$$
u(x, t)=\left[\mathcal{W}_{b d r}(t) h\right](x)=\left[U_{b}(t) h\right](x)+\overline{\left[U_{b}(t) h\right](x)}
$$

where, for $x, t \geq 0$,

$$
\left[U_{b}(t) h\right](x)=\frac{1}{2 \pi} \int_{1}^{\infty} e^{i t\left(\mu^{3}-\mu\right)} e^{-\left(\frac{\sqrt{3 \mu^{2}-4}+i \mu}{2}\right) x}\left(3 \mu^{2}-1\right) \int_{0}^{\infty} e^{-i \xi\left(\mu^{3}-\mu\right)} h(\xi) d \xi d \mu .
$$

Next, consider the same linear equation posed with zero boundary conditions, but non-trivial initial data, viz.

$$
\left.\begin{array}{ll}
u_{t}+u_{x}+u_{x x x}=0, & \text { for } x, t \geq 0 \\
u(x, 0)=\phi(x), & u(0, t)=0 .
\end{array}\right\}
$$

By semigroup theory, its solution may be obtained in the form

$$
u(t)=W_{c}(t) \phi
$$

where the spatial variable is suppressed and $W_{c}(t)$ is the $C_{0}$-semigroup in the space $L^{2}\left(R^{+}\right)$generated by the operator

$$
A f=-f^{\prime \prime \prime}-f^{\prime}
$$

with the domain

$$
\mathcal{D}(A)=\left\{f \in H^{3}\left(R^{+}\right) \mid f(0)=0\right\} .
$$

By d'Alembert's formula, one may use the semigroup $W_{c}(t)$ to formally write the solution of the forced linear problem

$$
\left.\begin{array}{l}
u_{t}+u_{x}+u_{x x x}=f, \quad \text { for } x, t \geq 0 \\
u(x, 0)=0, \quad u(0, t)=0,
\end{array}\right\}
$$

in the form

$$
u(\cdot, t)=\int_{0}^{t} W_{c}(t-\tau) f(\cdot, \tau) d \tau .
$$

The following helpful formula for $W_{c}(t) \phi$ was established in [4]. As is apparent from (2.4) and (2.6), this gives an explicit representation for solutions of the inhomogeneous problems (2.3) and (2.5).

Proposition 2.1. For any $\phi \in L^{2}\left(R^{+}\right)$, define

$$
\begin{gathered}
U_{0}^{+}(t) \phi(x)=\frac{1}{2 \pi} \int_{1}^{\infty} e^{i \mu^{3} t-i \mu t} \int_{0}^{\infty} e^{i \mu(x-\xi)} \phi(\xi) d \xi d \mu \\
U_{1}^{+}(t) \phi(x)=-\frac{1}{2 \pi} \int_{1}^{\infty} e^{i \mu^{3} t-i \mu t} e^{-\left(\frac{i \mu+\sqrt{3 \mu^{2}-4}}{2}\right) x} \int_{0}^{\infty} e^{-i \mu \xi} \phi(\xi) d \xi d \mu
\end{gathered}
$$

and

$$
U_{2}^{+}(t) \phi(x)=\frac{1}{2 \pi i} \int_{0}^{\infty} e^{-\mu^{3} t-\mu t} e^{-\left(\frac{\mu-i \sqrt{3 \mu^{2}+4}}{2}\right) x} \int_{0}^{\infty} e^{-\mu \xi} \phi(\xi) d \xi d \mu
$$


Then it follows that

$$
W_{c}(t) \phi(x)=\sum_{j=0}^{2}\left(U_{j}^{+}(t) \phi(x)+\overline{U_{j}^{+}(t) \phi(x)}\right) .
$$

As a comparison, recall the explicit solution formula for the pure initial-value problem (IVP) for the linear KdV equation (cf. (1.2))

$$
\left.\begin{array}{l}
u_{t}+u_{x}+u_{x x x}=0, \quad x, t \in R, \\
u(x, 0)=g(x), \quad x \in R,
\end{array}\right\}
$$

namely

$$
u(x, t)=W_{R}(t) g(x)=c \int_{-\infty}^{\infty} e^{i\left(\xi^{3}-\xi\right) t} e^{i x \xi} \int_{-\infty}^{\infty} e^{-i y \xi} g(y) d y d \xi .
$$

The formula for $W_{R}(t)$ is much simpler than that of $W_{c}(t)$. We take advantage of this simplicity to give a related representation of $W_{c}(t)$ in terms of $W_{R}(t)$ and $\mathcal{W}_{b d r}(t)^{2}$

Let a function $\phi$ be defined on the half line $R^{+}$and let $\phi^{*}$ be an extension of $\phi$ to the whole line $R$. The mapping $\phi \mapsto \phi^{*}$ can be organized so that it defines a bounded linear operator $B$ from $H^{s}\left(R^{+}\right)$to $H^{s}(R)$. Henceforth, $\phi^{*}=B \phi$ will refer to the result of such an extension operator applied to $\phi \in H^{s}\left(R^{+}\right)$. Assume that $v=v(x, t)$ is the solution of

$$
v_{t}+v_{x}+v_{x x x}=0, \quad v(x, 0)=\phi^{*}(x)
$$

for $x \in R, t \geq 0$. If $g(t)=v(0, t)$, then $v_{g}=v_{g}(x, t)=\mathcal{W}_{b d r}(t) g$ is the corresponding solution of the non-homogeneous boundary-value problem (2.1) with boundary condition $h(t)=g(t)$ for $t \geq 0$. It is clear that for $x>0$ the function $v(x, t)-v_{g}(x, t)$ solves the IBVP $(2.3)$, and this leads directly to a representation of the semigroup $W_{c}(t)$ in terms of $\mathcal{W}_{b d r}(t)$ and $W_{R}(t)$.

Proposition 2.2. For a given $s$ and $\phi \in H^{s}\left(R^{+}\right)$with $\phi(0)=0$, if $\phi^{*}$ is its extension to $R$ as described above, then $W_{c}(t) \phi$ may be written in the form

$$
W_{c}(t) \phi=W_{R}(t) \phi^{*}-\mathcal{W}_{b d r}(t) g
$$

for any $x, t>0$, where $g$ is the trace of $W_{R}(t) \phi^{*}$ at $x=0$.

Remark: This representation of $W_{c}(t)$ is less explicit than that presented in Proposition 2.1. However, it enables us to use the well established theory for $W_{R}(t)$ to study $W_{c}(t)$. It is worth emphasis, however, that $W_{c}$, by its nature, does not depend upon the extension $\phi^{*}$ of $\phi$. The representation in Proposition 2.2 does, of course, depend on the extension and this representation will be useful in deriving linear estimates.

In a similar manner, one may derive an alternative representation of solutions of the inhomogeneous initial-boundary-value problem (2.5).

Proposition 2.3. If $f^{*}(\cdot, t)=B f(\cdot, t)$ is an extension of $f$ from $R^{+} \times R^{+}$to $R \times R^{+}$, say, then the solution $u$ of (2.5) may be written in the form

$$
u(\cdot, t)=\int_{0}^{t} W_{R}(t-\tau) f^{*}(\cdot, \tau) d \tau-\mathcal{W}_{b d r}(t) v
$$

\footnotetext{
${ }^{2}$ This was suggested by one of the referees of our earlier paper [4].
} 
for any $x, t \geq 0$ where $v \equiv v(t)$ is the trace of $\int_{0}^{t} W_{R}(t-\tau) f^{*}(\cdot, \tau) d \tau$ at $x=0$.

Finally, consider the fully inhomogeneous initial-boundary-value problem

$$
\left.\begin{array}{ll}
u_{t}+u_{x}+u_{x x x}=f, & \text { for } x, t \geq 0, \\
u(x, 0)=\phi(x), & u(0, t)=h(t),
\end{array}\right\}
$$

where $\phi$ and $h$ are assumed to satisfy the compatibility condition $h(0)=\phi(0)$. Let $u(x, t)=z(x, t)+e^{-x-t} h(0)$. If $u$ solves $(2.9)$, then $z(x, t)$ solves

$$
\left.\begin{array}{l}
z_{t}+z_{x}+z_{x x x}=f+3 e^{-x-t} h(0), \quad \text { for } x, t \geq 0, \\
z(x, 0)=\phi(x)-e^{-x} \phi(0), \quad z(0, t)=h(t)-e^{-t} h(0) .
\end{array}\right\}
$$

Decompose $z$ in the form $z=w+v+y$ with

and

$$
\left.\begin{array}{c}
w_{t}+w_{x}+w_{x x x}=f+3 e^{-x-t} h(0), \quad \text { for } x, t \geq 0, \\
\begin{array}{c}
w(x, 0)=0, \\
w(0, t)=0,
\end{array} \\
v_{t}+v_{x}+v_{x x x}=0, \quad \text { for } x, t \geq 0, \\
v(x, 0)=\phi(x)-e^{-x} \phi(0), \quad v(0, t)=0,
\end{array}\right\}
$$

$$
\left.\begin{array}{l}
y_{t}+y_{x}+y_{x x x}=0, \quad \text { for } x, t \geq 0, \\
y(x, 0)=0, \quad y(0, t)=h(t)-e^{-t} h(0) .
\end{array}\right\}
$$

The following representation for the solution of (2.9) emerges from this decomposition together with the results of Propositions 2.3 and 2.4 and Duhamel's principle.

Proposition 2.4. The solution $u(x, t)$ of (2.9) may be realized in the form

$$
\begin{gathered}
u(x, t)=W_{c}(t)\left(\phi(x)-e^{-x} \phi(0)\right)+\int_{0}^{t} W_{c}(t-\tau)\left(f(x, \tau)+3 e^{-x-\tau} h(0)\right) d \tau+ \\
(2.10) \quad+\left[\mathcal{W}_{b d r}(t)\left(h(t)-e^{-t} h(0)\right)\right](x)+e^{-x-t} h(0) .
\end{gathered}
$$

In case $s \leq \frac{1}{2}$, the compatibility condition is not needed. One may choose $\phi(0)=h(0)=0$. In this situation, (2.10) becomes simply

$$
u(x, t)=W_{c}(t) \phi(x)+\int_{0}^{t} W_{c}(t-\tau) f(x, \tau) d \tau+\left[\mathcal{W}_{b d r}(t) h\right](x) .
$$

In any case, by Proposition 2.2 and Proposition 2.3, (2.10) may be written as

$$
\begin{aligned}
u(x, t)= & W_{R}(t) \phi_{1}^{*}(x)+\int_{0}^{t} W_{R}(t-\tau)\left(f^{*}(x, \tau)+3 p(x, \tau)\right) d \tau+ \\
& +\left[\mathcal{W}_{b d r}(t)\left(h(t)-e^{-t} h(0)-g(t)-v(t)\right)\right](x)+p(x, t)
\end{aligned}
$$

for $x \in R^{+}$and $t \geq 0$, where

$$
\phi_{1}(x)=\phi(x)-e^{-x} \phi(0), \quad p(x, t)=e^{-x-t} h(0)
$$

and $g(t)$ and $v(t)$ are the temporal traces of

$$
W_{R}(t) \phi_{1}^{*}(x) \text { and } \int_{0}^{t} W_{R}(t-\tau)\left(f^{*}(x, \tau)+3 p(x, \tau)\right) d \tau
$$


at $x=0$, respectively. Of course, if the right-hand side $f$ already happens to be defined on $R \times R$, there is no need to apply the extension operator.

The solution formula (2.12) holds only for $x>0$ and $t>0$. It will be convenient to extend this formula in such a way that it holds for all $x, t \in R$. This will provide a context in which to establish the well-posedness of the nonlinear problem in the framework of Bourgain spaces. Note that the first two terms on the right side of (2.12) are naturally defined for $x, t \in R$. Only the third term, viewed as a function of $x$ and $t$, needs to be extended from $R^{+} \times R^{+}$to $R \times R$. With an appropriate extension of the third term, to be denoted by $\left[\mathcal{W}_{b d r}^{*}(t)\left(h(t)-e^{-t} h(0)-g(t)-v(t)\right)\right](x)$, the function $u(x, t)$ given by the formula (2.12) may be viewed as a function of $x$ defined on the whole line $R$, which of course solves (2.9) when restricted to $R^{+} \times R^{+}$. If one replaces $f$ in $(1.12)$ by $-u u_{x}$ and drops the extension operator, there appears the nonlinear integral equation

$$
\begin{aligned}
u(x, t)= & W_{R}(t) \phi_{1}^{*}(x)-\int_{0}^{t} W_{R}(t-\tau) u(x, \tau) u_{x}(x, \tau) d \tau+ \\
& +3 \int_{0}^{t} W_{R}(t-\tau) p(x, \tau) d \tau+ \\
& {\left[\mathcal{W}_{b d r}^{*}(t)\left(h(t)-e^{-t} h(0)-g(t)-v(t)\right)\right](x)+p(x, t) }
\end{aligned}
$$

posed on the whole plane $R \times R$. It is clear from its construction that if this integral equation has a solution, then when the solution is restricted to the domain $R^{+} \times(0, T)$, it solves the IBVP (1.1). It is also clear that if $u \in C\left(0, T ; H^{3}\left(R^{+}\right)\right)$, then it is a strong solution (distributional solution for which all of the terms in the equation lie in $C\left(0, T ; L^{2}\left(R^{+}\right)\right)$and such that they sum to the zero function in this latter space).

\subsection{Extensions of the boundary integral operator}

As pointed out in Section 2.1, to use the Bourgain spaces in a straightforward way to study well-posedness of the IBVP (1.1), one needs to extend the boundary integral operator $\mathcal{W}_{b d r}(t)$ to an integral operator $\mathcal{W}_{b d r}^{*}(t)$ so that, for any given boundary value function $h(t),\left[\mathcal{W}_{b d r}^{*}(t) h(t)\right](x)$ is, for each $t \in R$, a function of $x$ defined on the whole line $R$. There are infinitely many ways to accomplish such an extension; among them the even extension is probably the simplest. However, as will be made clear presently, and especially in Appendix II, special extensions are needed if one intends to capture some of the more subtle smoothing properties induced via the imposition of a boundary condition at $x=0$.

Next are presented three different types of extensions of the boundary integral operator $\mathcal{W}_{b d r}(t)$. Rewrite $\mathcal{W}_{b d r}(t)$ as

$$
\begin{aligned}
& {\left[\mathcal{W}_{b d r}(t) h\right](x)} \\
& =\frac{1}{2 \pi} R e \int_{1}^{\infty} e^{i \mu^{3} t-i \mu t} e^{-\left(\sqrt{3 \mu^{2}-4}+i \mu\right) \frac{x}{2}}\left(3 \mu^{2}-1\right) \int_{0}^{\infty} e^{-i\left(\mu^{3}-\mu\right) \xi} h(\xi) d \xi d \mu \\
& =\frac{1}{2 \pi} R e \int_{1}^{4} e^{i \mu^{3} t-i \mu t} e^{-\left(\sqrt{3 \mu^{2}-4}+i \mu\right) \phi_{3}(x)}\left(3 \mu^{2}-1\right) \phi_{1}(\mu) \int_{0}^{\infty} e^{-i\left(\mu^{3}-\mu\right) \xi} h(\xi) d \xi d \mu \\
& +\frac{1}{2 \pi} R e \int_{\frac{2}{\sqrt{3}}}^{\infty} e^{i \mu^{3} t-i \mu t} e^{-\left(\sqrt{3 \mu^{2}-4}+i \mu\right) \frac{x}{2}}\left(3 \mu^{2}-1\right) \phi_{2}(\mu) \int_{0}^{\infty} e^{-i\left(\mu^{3}-\mu\right) \xi} h(\xi) d \xi d \mu \\
& :=\frac{1}{2 \pi}\left\{I_{1}(x, t)+I_{2}(x, t)\right\}
\end{aligned}
$$


where $\phi_{1}(\mu)$ and $\phi_{2}(\mu)$ are nonnegative cut-off functions satisfying

$$
\phi_{1}(\mu)+\phi_{2}(\mu)=1 \quad \text { for any } \mu \in R^{+}
$$

with supp $\phi_{1} \subset(-1,4)$, supp $\phi_{2} \subset(3, \infty)$ and $\phi_{3}(x)$ is a smooth function on $R$ such that

$$
\phi_{3}(x)=\frac{x}{2} \quad \text { for } x \geq 0, \quad \phi_{3}(x)=0 \quad \text { for } x \leq-1 .
$$

Observe that

$$
\frac{\sqrt{3 \mu^{2}-4}+i \mu}{2} x=\frac{\sqrt{4-3 \mu^{2}}+\mu}{2} i x
$$

is purely imaginary for $1 \leq \mu \leq 2 / \sqrt{3}$. The integral $I_{1}$ is naturally defined for all values of $x$ and $t$ and, viewed as a function defined on $R \times R$, is in fact $C^{\infty}$-smooth there, with all its derivatives decreasing rapidly as $x \rightarrow \pm \infty$. Thus no complicated extension of $I_{1}$ is required as the obvious one suffices. It is otherwise for $I_{2}$. To discuss $I_{2}$, it is convenient to let $\mu(\lambda)$ denote the positive solution of

$$
\mu^{3}-\mu=\lambda
$$

for $\lambda \geq 0$ and $\mu \geq 1$, while $\mu(\lambda)=-\mu(-\lambda)$ for $\lambda<0$. Note that $\mu(\lambda)$ is strictly increasing on $[0, \infty)$ and that values of $\mu \geq \frac{2}{\sqrt{3}}$ correspond to values of $\lambda \geq \frac{2}{3 \sqrt{3}}$. By a change of variables, the integral $I_{2}$ can be rewritten in the form

$$
\begin{aligned}
I_{2}(x, t) & =\operatorname{Re} \int_{\frac{2}{3 \sqrt{3}}}^{\infty} \int_{0}^{\infty} e^{i \lambda t} e^{-\left(\sqrt{3 \mu^{2}(\lambda)-4}+i \mu(\lambda)\right) \frac{x}{2}} e^{-i \lambda s} \phi_{2}(\mu(\lambda)) h(s) d s d \lambda \\
& =\int_{\frac{2}{3 \sqrt{3}}}^{\infty} \int_{0}^{\infty} e^{-\frac{x \sqrt{3 \mu^{2}(\lambda)-4}}{2}} \cos \left(\lambda(t-s)-\frac{1}{2} \mu(\lambda) x\right) \phi_{2}(\mu(\lambda)) h(s) d s d \lambda \\
& :=E(x, t)
\end{aligned}
$$

for $x \geq 0$. Let the extension of $E(x, t)$ to $x<0$ be $g(x, t)$ and write

$$
I_{2}(x, t)= \begin{cases}E(x, t), & x \geq 0, \\ g(x, t), & x<0,\end{cases}
$$

where $g(x, t)$ is to be defined. Note that

$$
\begin{gathered}
\mathcal{F}_{x}\left[I_{2}\right](\xi, t)=\int_{-\infty}^{\infty} I_{2}(x, t) e^{i x \xi} d x=\int_{-\infty}^{0} g(x, t)(\cos (x \xi)+i \sin (x \xi)) d x \\
+\int_{0}^{\infty} E(x, t)(\cos (x \xi)+i \sin (x \xi)) d x \\
=\int_{0}^{\infty}(E(x, t) \cos (x \xi)+g(-x, t) \cos (x \xi)) d x \\
+i \int_{0}^{\infty}(E(x, t) \sin (x \xi)-g(-x, t) \sin (x \xi)) d x .
\end{gathered}
$$

Recall the identities

$$
\int_{0}^{\infty} \sin (x \xi) \int_{0}^{\infty} \cos (x \eta) h(\eta) d \eta d x=\frac{1}{2} \int_{-\infty}^{\infty} \frac{1}{\xi-x} h(x) d x
$$


and

$$
h(x)=\frac{2}{\pi} \int_{0}^{\infty} \cos (x \xi) \int_{0}^{\infty} \cos (\xi \eta) h(\eta) d \eta d \xi,
$$

where $h(x)$ is extended evenly to negative values of $x$. These relations yield

$$
\begin{gathered}
\int_{0}^{\infty}(E(x, t) \sin (x \xi)-g(-x, t) \sin (x \xi)) d x \\
=\frac{1}{\pi} \int_{-\infty}^{\infty} \frac{1}{\xi-\eta}\left(\int_{0}^{\infty} \cos (\eta x) E(x, t) d x-\int_{0}^{\infty} \cos (\eta x) g(-x, t) d x\right) d \eta .
\end{gathered}
$$

In consequence, it transpires that

$$
\begin{aligned}
\mathcal{F}_{x, t}\left[I_{2}\right]= & \mathcal{F}_{t}\left[\int_{0}^{\infty}(E(x, t) \cos (x \xi)+g(-x, t) \cos (x \xi)) d x\right] \\
& +\frac{i}{\pi} \int_{-\infty}^{\infty} \frac{1}{\xi-\eta} \mathcal{F}_{t}\left[\int_{0}^{\infty} \cos (\eta x) E(x, t) d x-\int_{0}^{\infty} \cos (\eta x) g(-x, t) d x\right] d \eta .
\end{aligned}
$$

Note that different choices of $g(x, t)$ give different extensions of $E(x, t)$ to $x<0$. The following three choices of $g(x, t)$ will be studied in this article.

(i) Let $g(-x, t)=E(x, t)$ for $x \in R^{+}$. This is perhaps the simplest extension. It results in the formula

$$
\mathcal{F}_{x, t}\left[I_{2}\right]=\mathcal{F}_{t}\left[2 \int_{0}^{\infty} E(x, t) \cos (x \xi) d x\right] .
$$

The boundary integral operator corresponding to this extension of $\mathcal{W}_{b d r}(t)$ is denoted by $\mathcal{B} I_{e}(t)$; the subscript $e$ stands for even of course.

(ii) For $x>0$, choose $g(-x, t)$ such that

$$
\begin{gathered}
\mathcal{F}_{t}\left[\int_{0}^{\infty} g(-x, t) \cos (x \xi) d x\right](\tau)=-\mathcal{F}_{t}\left[\int_{0}^{\infty} E(x, t) \cos (x \xi) d x\right](\tau) \Theta(\xi, \tau) \\
\quad+\mathcal{F}_{t}\left[\int_{0}^{\infty} E(x, t) \cos (x \xi) d x\right](\tau)(1-\Theta(\xi, \tau)) \nu(\xi) \omega(\tau)
\end{gathered}
$$

where $\Theta(\xi, \tau)=\chi\left(|\xi|-\delta|\tau|^{1 / 3}\right)$ with $\delta>0$ fixed, $0 \leq \chi(\xi) \leq 1$ everywhere, and

$$
\chi(\xi)= \begin{cases}1, & \xi<0, \\ 0, & \xi>1,\end{cases}
$$

whilst

$$
\nu(\xi)= \begin{cases}-1, & |\xi| \geq 1, \\ 0, & |\xi|<1,\end{cases}
$$

and $\omega(\tau)$ is a smooth and bounded function to be specified momentarily. It is easy to see that such a $g$ is a combination of even and odd extension, viz.

$$
\mathcal{F}_{x, t}\left[I_{2}\right]:=\hat{I}_{21}(\xi, \tau)+\hat{I}_{22}(\xi, \tau)
$$

where

$$
\hat{I}_{21}(\xi, \tau)=\mathcal{F}_{t}\left[\int_{0}^{\infty} E(x, t) \cos (x \xi) d x\right](\tau)(1-\Theta(\xi, \tau))(1+\nu(\xi) \omega(\tau))
$$


and

$$
\begin{aligned}
\hat{I}_{22}(\xi, \tau) & =\frac{i}{\pi} \int_{-\infty}^{\infty} \frac{1}{\xi-\eta} \mathcal{F}_{t}\left[\int_{0}^{\infty} E(x, t) \cos (x \eta) d x\right](\tau) \\
& \times[2 \Theta(\eta, \tau)+(1-\Theta(\eta, \tau))(1-\nu(\eta) \omega(\tau))] d \eta \\
=\frac{i}{\pi} \int_{0}^{\infty} & \left(\frac{1}{\xi-\eta}+\frac{1}{\xi+\eta}\right) \mathcal{F}_{t}\left[\int_{0}^{\infty} E(x, t) \cos (x \eta) d x\right](\tau) \\
& \times[2 \Theta(\eta, \tau)+(1-\Theta(\eta, \tau))(1-\nu(\eta) \omega(\tau))] d \eta .
\end{aligned}
$$

Because of the algebraic identity

$$
\frac{1}{\xi-\eta}+\frac{1}{\xi+\eta}=\frac{2}{\xi}\left(1+\frac{\eta^{2}}{\xi^{2}-\eta^{2}}\right)
$$

we may write $\hat{I}_{22}(\xi, \tau)$ as

$$
\begin{aligned}
\hat{I}_{22}(\xi, \tau)= & \frac{2 i}{\pi \xi} \int_{0}^{\infty} \mathcal{F}_{t}\left[\int_{0}^{\infty} E(x, t) \cos (x \eta) d x\right] \\
& \times[2 \Theta(\eta, \tau)+(1-\Theta(\eta, \tau))(1-\nu(\xi) \omega(\tau))] d \eta \\
& +\frac{2 i}{\pi \xi} \int_{0}^{\infty} \frac{(\eta / \xi)^{2}}{1-(\eta / \xi)^{2}} \mathcal{F}_{t}\left[\int_{0}^{\infty} E(x, t) \cos (x \eta) d x\right] \\
& \times[2 \Theta(\eta, \tau)+(1-\Theta(\eta, \tau))(1-\nu(\xi) \omega(\tau))] d \eta \\
:= & Q_{1}(\xi, \tau)+Q_{2}(\xi, \tau) .
\end{aligned}
$$

Rewrite the integral $E(x, t)$ as follows;

$$
\begin{gathered}
E(x, t)=\operatorname{Re} \int_{\frac{2}{3 \sqrt{3}}}^{\infty} \int_{0}^{\infty} e^{i \lambda t} e^{-\left(\sqrt{3 \mu^{2}(\lambda)-4}+i \mu(\lambda)\right) \frac{x}{2}} e^{-i \lambda s} \phi_{2}(\mu(\lambda)) h(s) d s d \lambda \\
=\frac{1}{2} \int_{\frac{2}{3 \sqrt{3}}}^{\infty} \int_{0}^{\infty} e^{i \lambda t} e^{-\left(\sqrt{3 \mu^{2}(\lambda)-4}+i \mu(\lambda)\right) \frac{x}{2}} e^{-i \lambda s} \phi_{2}(\mu(\lambda)) h(s) d s d \lambda \\
+\frac{1}{2} \int_{\frac{2}{3 \sqrt{3}}}^{\infty} \int_{0}^{\infty} e^{-i \lambda t} e^{-\left(\sqrt{3 \mu^{2}(\lambda)-4}-i \mu(\lambda)\right) \frac{x}{2}} e^{i \lambda s} \phi_{2}(\mu(\lambda)) h(s) d s d \lambda \\
=\frac{1}{2} \int_{-\infty}^{\infty} \int_{0}^{\infty} e^{i \lambda t} e^{-\left(\sqrt{3 \mu^{2}(\lambda)-4}+i \mu(\lambda)\right) \frac{x}{2}} e^{-i \lambda s} \phi_{2}(\mu(\lambda)) h(s) d s d \lambda \\
+\frac{1}{2} \int_{-\infty}^{\infty} \int_{0}^{\infty} e^{-i \lambda t} e^{-\left(\sqrt{3 \mu^{2}(\lambda)-4}-i \mu(\lambda)\right) \frac{x}{2}} e^{i \lambda s} \phi_{2}(\mu(\lambda)) h(s) d s d \lambda
\end{gathered}
$$


where the fact that $\phi_{2}(\mu(\lambda))=0$ for $\lambda<\frac{2}{3 \sqrt{3}}$ was used in the last step, a point that follows since supp $\phi_{2} \subset(3, \infty)$. A direct computation reveals that

$\int_{0}^{\infty} e^{-\left(\sqrt{3 \mu^{2}(\lambda)-4}+i \mu(\lambda)\right) \frac{x}{2}} \cos (x \eta) d x=K_{11}(\eta, \lambda)+K_{21}(\eta, \lambda)+K_{31}(\eta, \lambda)+K_{41}(\eta, \lambda)$ and

$\int_{0}^{\infty} e^{-\left(\sqrt{3 \mu^{2}(\lambda)-4}-i \mu(\lambda)\right) \frac{x}{2}} \cos (x \eta) d x=K_{12}(\eta, \lambda)+K_{22}(\eta, \lambda)+K_{32}(\eta, \lambda)+K_{42}(\eta, \lambda)$

with

$$
K_{11}(\eta, \lambda)=\frac{\sqrt{3 \mu^{2}(\lambda)-4}}{3 \mu^{2}(\lambda)-4+(2 \eta+\mu(\lambda))^{2}}
$$

$$
K_{21}(\eta, \lambda)=\frac{\sqrt{3 \mu^{2}(\lambda)-4}}{3 \mu^{2}(\lambda)-4+(2 \eta-\mu(\lambda))^{2}}
$$

$$
\begin{aligned}
& K_{31}(\eta, \lambda)=\frac{\left(3 \mu^{2}(\lambda)-4\right) \mu(\lambda)}{2 i\left(3 \mu^{2}(\lambda)-4+(2 \eta+\mu(\lambda))^{2}\right)\left(3 \mu^{2}(\lambda)-4+(2 \eta-\mu(\lambda))^{2}\right)} \\
& K_{41}(\eta, \lambda)=\frac{\left(\mu^{2}(\lambda)-4 \eta^{2}\right) \mu(\lambda)}{2 i\left(3 \mu^{2}(\lambda)-4+(2 \eta+\mu(\lambda))^{2}\right)\left(3 \mu^{2}(\lambda)-4+(2 \eta-\mu(\lambda))^{2}\right)}
\end{aligned}
$$

and

$$
\left\{\begin{array}{l}
K_{12}(\eta, \lambda)=K_{11}(\eta, \lambda), K_{22}(\eta, \lambda)=K_{21}(\eta, \lambda), \\
K_{32}(\eta, \lambda)=-K_{31}(\eta, \lambda), K_{42}(\eta, \lambda)=-K_{41}(\eta, \lambda) .
\end{array}\right.
$$

Thus, $\mathcal{F}_{t}\left[\int_{0}^{\infty} E(x, t) \cos (x \eta) d x\right]$ may be expressed in the form

$$
\begin{gathered}
\mathcal{F}_{t}\left[\int_{0}^{\infty} E(x, t) \cos (x \eta) d x\right](\tau) \\
=\sum_{m=1}^{4} K_{m 1}(\eta, \tau) \phi_{2}(\mu(\tau)) \hat{h}(\tau)+\sum_{m=1}^{4} K_{m 2}(\eta,-\tau) \phi_{2}(\mu(-\tau)) \hat{h}(-\tau)
\end{gathered}
$$

where

$$
\hat{h}(\tau)=\int_{0}^{\infty} e^{-i \tau s} h(s) d s .
$$

Here, we note that from the definition of $\phi_{2}$, for any given $\tau$ there is only one nonzero summand in (2.21) and the sum involving the terms $K_{m 2}(\eta,-\tau)$ is the same as the sum involving on the terms $K_{m 1}(\eta, \tau)$. Because $\phi_{2}(\mu(\tau))$ is a bounded $C^{\infty}$-function, and since, as direct calculation shows,

$$
\left|\int_{0}^{\infty} \sum_{m=1}^{4} K_{m 1}(\eta, \tau)(1-\Theta(\eta, \tau)) d \eta\right| \geq C_{2}>0
$$


where $C_{2}$ is a fixed constant independent of $\tau$, it follows that the formula,

$$
\begin{gathered}
(1+\omega(\tau)) \phi_{2}(\mu(\tau)) \int_{0}^{\infty} \sum_{m=1}^{4} K_{m 1}(\eta, \tau)(1-\Theta(\eta, \tau)) d \eta \\
=-2 \phi_{2}(\mu(\tau)) \int_{0}^{\infty} \sum_{m=1}^{4} K_{m 1}(\eta, \tau) \Theta(\eta, \tau) d \eta
\end{gathered}
$$

defines the $C^{\infty}(R)$-function $\omega(\tau)$ in such a way that $d^{k} \omega / d \tau^{k}$ is bounded on $R$, for $k=0,1,2, \cdots$. It is clear that this choice of $\omega(\tau)$ makes

$$
Q_{1}(\xi, \tau) \equiv 0, \quad \text { for all } \tau \text { when }|\xi| \geq 1
$$

Hence, for $|\xi| \geq 1$,

$$
\begin{aligned}
& \hat{I}_{22}(\xi, \tau)=Q_{2}(\xi, \tau) \\
= & \frac{2 i}{\pi \xi} \int_{0}^{\infty} \frac{\eta^{2}}{\xi^{2}-\eta^{2}} \mathcal{F}_{t}\left[\int_{0}^{\infty} E(x, t) \cos (x \eta) d x\right](\tau) \Theta_{1}(\eta, \tau) d \eta
\end{aligned}
$$

where

$$
\Theta_{1}(\eta, \tau)=2 \Theta(\eta, \tau)+(1-\Theta(\eta, \tau))(1-\nu(\eta) \omega(\tau)) .
$$

Moreover, when $|\xi| \geq 1$ and $\tau \geq 0$,

$$
\hat{I}_{22}(\xi, \tau)=\frac{2 i}{\pi \xi} \int_{0}^{\infty} \frac{\eta^{2}}{\xi^{2}-\eta^{2}}\left[\sum_{m=1}^{4} K_{m 1}(\eta, \tau) \phi_{2}(\mu(\tau)) \hat{h}(\tau)\right] \Theta_{1}(\eta, \tau) d \eta,
$$

whereas

$$
\hat{I}_{22}(\xi, \tau)=\frac{2 i}{\pi \xi} \int_{0}^{\infty} \frac{\eta^{2}}{\xi^{2}-\eta^{2}}\left[\sum_{m=1}^{4} K_{m 2}(\eta,-\tau) \phi_{2}(\mu(-\tau)) \hat{h}(-\tau)\right] \Theta_{1}(\eta, \tau) d \eta
$$

when $|\xi| \geq 1$ and $\tau<0$. The boundary integral operator corresponding to this extension of $\mathcal{W}_{b d r}(t)$ is denoted by $\mathcal{B} I_{m 1}(t)$.

(iii) For $x>0$, choose $g(-x, t)$ such that

$$
\begin{aligned}
& \mathcal{F}_{t}\left[\int_{0}^{\infty} g(-x, t) \cos (x \xi) d x\right](\tau)=-\mathcal{F}_{t}\left[\int_{0}^{\infty} E(x, t) \cos (x \xi)\right](\tau)(1-\Theta(\xi, \tau)) \\
& \quad+\mathcal{F}_{t}\left[\int_{0}^{\infty} E(x, t) \cos (x \xi) d x\right](\tau) \Theta(\xi, \tau) \nu(\xi) \omega(\tau) .
\end{aligned}
$$

In this case,

$$
\mathcal{F}_{x, t}\left[I_{2}\right]:=\hat{I}_{21}^{*}(\xi, \tau)+\hat{I}_{22}^{*}(\xi, \tau)
$$

where, if $\Theta_{2}(\eta, \tau)=2(1-\Theta(\eta, \tau))+\Theta(\eta, \tau)(1-\nu(\eta) \omega(\tau))$, then

$$
\hat{I}_{21}^{*}(\xi, \tau)=\mathcal{F}_{t}\left[\int_{0}^{\infty} E(x, t) \cos (x \xi) d x\right](\tau) \Theta(\xi, \tau)(1+\nu(\xi) \omega(\tau))
$$


and

$$
\begin{aligned}
\hat{I}_{22}^{*}(\xi, \tau)= & \frac{i}{\pi} \int_{-\infty}^{\infty} \frac{1}{\xi-\eta} \mathcal{F}_{t}\left[\int_{0}^{\infty} E(x, t) \cos (x \eta) d x\right](\tau) \Theta_{2}(\eta, \tau) d \eta \\
= & \frac{i}{\pi} \int_{0}^{\infty}\left(\frac{1}{\xi-\eta}+\frac{1}{\xi+\eta}\right) \mathcal{F}_{t}\left[\int_{0}^{\infty} E(x, t) \cos (x \eta) d x\right](\tau) \Theta_{2}(\eta, \tau) d \eta \\
= & \frac{2 i}{\pi \xi} \int_{0}^{\infty} \mathcal{F}_{t}\left[\int_{0}^{\infty} E(x, t) \cos (x \eta) d x\right](\tau) \Theta_{2}(\eta, \tau) d \eta \\
& \quad+\frac{2 i}{\pi \xi} \int_{0}^{\infty} \frac{(\eta / \xi)^{2}}{1-(\eta / \xi)^{2}} \mathcal{F}_{t}\left[\int_{0}^{\infty} E(x, t) \cos (x \eta) d x\right](\tau) \Theta_{2}(\eta, \tau) d \eta \\
:= & Q_{1}^{*}(\xi, \tau)+Q_{2}^{*}(\xi, \tau) .
\end{aligned}
$$

Just as in case (ii), one can choose an appropriate function $\omega(\tau)$ such that

$$
Q_{1}^{*}(\xi, \tau)=0 \quad \text { for }|\xi|>1 \text { and any } \tau \in R .
$$

The boundary integral operator corresponding to this extension of $\mathcal{W}_{b d r}(t)$ is denoted by $\mathcal{B} I_{m 2}(t)$.

\section{Boundary smoothing properties}

In this section, attention is focused upon the non-homogeneous boundaryvalue problem

$$
\left.\begin{array}{l}
u_{t}+u_{x}+u_{x x x}=0, \quad \text { for } x, t \geq 0, \\
u(x, 0)=0, \quad u(0, t)=h(t) .
\end{array}\right\}
$$

Our analysis turns around a detailed understanding of the boundary integral operators introduced in Section 2. define

For given $s \in R, 0 \leq b \leq 1, \alpha>\frac{1}{2}$ and any function $w \equiv w(x, t): R \times R \rightarrow R$,

$$
\begin{aligned}
& \Lambda_{s, b}(w)=\left(\int_{-\infty}^{\infty} \int_{-\infty}^{\infty}\left(1+\left|\tau-\left(\xi^{3}-\xi\right)\right|\right)^{2 b}(1+|\xi|)^{2 s}|\hat{w}(\xi, \tau)|^{2} d \xi d \tau\right)^{\frac{1}{2}} \\
& \lambda_{\alpha}(w)=\left(\int_{-\infty}^{\infty} \int_{|\xi| \leq 1}(1+|\tau|)^{2 \alpha}|\hat{w}(\xi, \tau)|^{2} d \xi d \tau\right)^{\frac{1}{2}}
\end{aligned}
$$

Consider first the operator $\mathcal{B} I_{m 1}(t)$.

THEOREM 3.1. Let $\psi(t)$ be a given smooth function of $t$ with compact support and assume that $s$ and $b$ are within the range $0 \leq b<\frac{1}{2}+\frac{s}{3}<1$. Then there exists a constant $C$ depending only on $\psi$ such that

$$
\Lambda_{-s, b}\left(\psi \mathcal{B} I_{m 1}(h)\right) \leq C\|h\|_{H} \frac{3 b-s-1 / 2}{3}\left(R^{+}\right)
$$

for any $h \in H_{0}^{\frac{3 b-s-1 / 2}{3}}\left(R^{+}\right)$. 
Proof: Recall that

$$
\left[\mathcal{B} I_{m 1}(t) h\right](x)=I_{1}(x, t)+I_{2}(x, t)
$$

where $I_{1}(x, t)$ is a function defined on the whole plane $R \times R$ and is, in fact, a $C^{\infty}$-smooth function of $x$ and $t$. For any $t \in R$,

$$
\begin{aligned}
\left\|I_{1}(x, t)\right\|_{L_{x}^{2}(R)} & \leq C\left\|\left(3 \mu^{2}-1\right) \phi_{1}(\mu) \int_{0}^{\infty} e^{-i\left(\mu^{3}-\mu\right) \xi} h(\xi) d \xi\right\|_{L_{\mu}^{2}(R)} \\
& \leq C\|h\|_{L^{2}\left(R^{+}\right)} .
\end{aligned}
$$

This type of inequality is also valid for $\partial_{x}^{j} \partial_{t}^{l} I_{1}$ for any $j, l \geq 0$. Thus it is straightforward to see that if $h \in L^{2}\left(R^{+}\right)$, then

$$
\Lambda_{-s, b}\left(\psi I_{1}\right) \leq C\|h\|_{L^{2}\left(R^{+}\right)}
$$

for any given $b \geq 0$ and $s \in R$ where the constant $C$ depends only on $\psi, b$ and $s$.

To analyze $I_{2}(x, t)$, remember that

$$
\mathcal{F}_{x, t}\left[I_{2}\right](\xi, \tau)=\hat{I}_{21}(\xi, \tau)+\hat{I}_{22}(\xi, \tau)
$$

where, for $|\xi| \geq 1$,

$$
\hat{I}_{21}(\xi, \tau)=\mathcal{F}_{t}\left[\int_{0}^{\infty} E(x, t) \cos (x \xi) d x\right](\tau)(1-\Theta(\xi, \tau))(1+\omega(\tau))
$$

and

$$
\hat{I}_{22}(\xi, \tau)=\frac{i}{\pi} \int_{0}^{\infty}\left(\frac{1}{\xi-\eta}+\frac{1}{\xi+\eta}\right) \mathcal{F}_{t}\left[\int_{0}^{\infty} E(x, t) \cos (x \eta) d x\right](\tau) \Theta_{1}(\eta, \tau) d \eta
$$

Since the relevant estimates in the region $|\xi|<1$ are straightforward, in what follows it is always assumed that $|\xi| \geq 1$. First, consider the term

$$
\int_{-\infty}^{\infty} \int_{-\infty}^{\infty}\left(1+\left|\tau-\left(\xi^{3}-\xi\right)\right|\right)^{2 b}(1+|\xi|)^{-2 s}\left|\hat{I}_{21}(\xi, \tau)\right|^{2} d \xi d \tau
$$

We have the following estimate for this term.

Proposition 3.2. Let $s \geq 0$ and $0<b<\frac{1}{2}+\frac{s}{3}$ be given. There exists $a$ constant $C$ such that

$$
\int_{-\infty}^{\infty} \int_{-\infty}^{\infty}\left(1+\left|\tau-\left(\xi^{3}-\xi\right)\right|\right)^{2 b}(1+|\xi|)^{-2 s}\left|\hat{I}_{21}(\xi, \tau)\right|^{2} d \xi d \tau \leq C\|h\|_{H^{\frac{3 b-s-1 / 2}{3}}}^{2}\left(R^{+}\right)
$$

for any $h \in H^{\frac{3 b-s-1 / 2}{3}}\left(R^{+}\right)$.

Proof of Proposition 3.2: According to (2.21),

$$
\begin{gathered}
\mathcal{F}_{t}\left[\int_{0}^{\infty} E(x, t) \cos (x, \xi) d x\right](\tau) \\
=\sum_{m=1}^{4} K_{m 1}(\xi, \tau) \phi_{2}(\mu(\tau)) \hat{h}(\tau)+\sum_{m=1}^{4} K_{m 2}(\xi,-\tau) \phi_{2}(\mu(-\tau)) \hat{h}(-\tau) .
\end{gathered}
$$

In the following, detailed analysis is given for terms containing $K_{21}$ and $K_{41}$; the estimates for the other terms follow similar lines. Suppose that $\xi \geq 0$. The case $\xi<0$ is entirely analogous. Write

$$
A_{m 1}(\xi, \tau)=K_{m 1}(\xi, \tau) \phi_{2}(\mu(\tau)) \hat{h}(\tau), m=1,2,3,4
$$


For given $s$ and $b>0$, we have

$$
\begin{aligned}
& \int_{-\infty}^{\infty} \int_{0}^{\infty}\left(1+\left|\tau-\left(\xi^{3}-\xi\right)\right|\right)^{2 b}(1+|\xi|)^{-2 s}\left|A_{21}(\xi, \tau)(1+\omega(\tau))(1-\Theta(\xi, \tau))\right|^{2} d \xi d \tau \\
& \leq C \int_{-\infty}^{\infty} \phi_{2}(\mu(\tau))\left|\int_{0}^{\infty} h(s) e^{-i s \tau} d s\right|^{2} B_{21}(\tau) d \tau
\end{aligned}
$$

with

$$
\begin{aligned}
B_{21}(\tau)=\int_{0}^{\infty}(1+ & \left.\left|\tau-\left(\xi^{3}-\xi\right)\right|\right)^{2 b}(1+|\xi|)^{-2 s}|(1+\omega(\tau))(1-\Theta(\xi, \tau))|^{2} \\
& \times \frac{\left(3 \mu^{2}(\tau)-4\right) \phi_{2}(\mu(\tau))}{\left(3 \mu^{2}(\tau)-4+(2 \xi-\mu(\tau))^{2}\right)^{2}} d \xi
\end{aligned}
$$

Claim: If $b<\frac{1}{2}+\frac{s}{3}$, then, as $\tau \rightarrow \infty$,

$$
B_{21}(\tau)=O\left(\tau^{\frac{6 b-2 s-1}{3}}\right)
$$

To see the claim is true, note that in fact

$$
\begin{gathered}
B_{21}(\tau)=\int_{\delta|\tau|^{\frac{1}{3}}}^{\infty}\left(1+\left|\tau-\left(\xi^{3}-\xi\right)\right|\right)^{2 b}(1+|\xi|)^{-2 s} \frac{\left(3 \mu^{2}(\tau)-4\right) \phi_{2}(\mu(\tau))}{\left(3 \mu^{2}(\tau)-4+(2 \xi-\mu(\tau))^{2}\right)^{2}} \\
\times(1+\omega(\tau))^{2}(1-\Theta(\xi, \tau))^{2} d \xi
\end{gathered}
$$

since $\Theta(\xi, \tau)=1$ when $\xi<\delta|\tau|^{\frac{1}{3}}$, where $\delta>0$ is fixed, but arbitrary for the nonce (see $(2.14))$. Let $\xi=\eta(\zeta)$ be the real solution of the equation

$$
\xi^{3}-\xi=\zeta, \quad \text { for } \quad 0 \leq \zeta<\infty
$$

that connects continuously to the unique real root as $\zeta$ becomes large (e.g. $\zeta>\frac{2}{3 \sqrt{3}}$ ). Note that

$$
\eta(\zeta) \sim \zeta^{\frac{1}{3}} \quad \text { as } \quad \zeta \rightarrow \infty
$$


For large $\tau$, it is also the case that $\mu(\tau) \sim \tau^{\frac{1}{3}}$. Thus, for $\tau>0$ large enough,

$$
\begin{aligned}
B_{21}(\tau) & \leq C \int_{\delta^{3} \tau}^{\infty} \frac{\left(3 \mu^{2}(\tau)-4\right)(1+|\tau-\zeta|)^{2 b}}{\left(3 \mu^{2}(\tau)-4+(2 \eta(\zeta)-\mu(\tau))^{2}\right)^{2}}(1+|\zeta|)^{-\frac{2 s}{3}} \frac{1}{3 \eta^{2}(\zeta)-1} d \zeta \\
& \leq C \int_{\delta^{3} \tau}^{\infty} \frac{\tau^{\frac{2}{3}}(1+|\tau-\zeta|)^{2 b}}{\left(1+3 \tau^{\frac{2}{3}}+\left(2 \eta(\zeta)-\tau^{\frac{1}{3}}\right)^{2}\right)^{2}}(1+|\zeta|)^{-\frac{2 s}{3}} \frac{1}{3 \eta^{2}(\zeta)-1} d \zeta \\
& =C \int_{\delta^{3} \tau}^{2 \tau} \frac{\tau^{\frac{2}{3}}(1+|\tau-\zeta|)^{2 b}}{\left(1+3 \tau^{\frac{2}{3}}+\left(2 \eta(\zeta)-\tau^{\frac{1}{3}}\right)^{2}\right)^{2}}(1+|\zeta|)^{-\frac{2 s}{3}} \frac{1}{3 \eta^{2}(\zeta)-1} d \zeta \\
& +C \int_{2 \tau}^{\infty} \frac{\tau^{\frac{2}{3}}(1+|\tau-\zeta|)^{2 b}}{\left(1+3 \tau^{\frac{2}{3}}+\left(2 \eta(\zeta)-\tau^{\frac{1}{3}}\right)^{2}\right)^{2}}(1+|\zeta|)^{-\frac{2 s}{3}} \frac{1}{3 \eta^{2}(\zeta)-1} d \zeta \\
& :=G_{21-1}(\tau)+G_{21-2}(\tau) .
\end{aligned}
$$

Continuing this sequence of inequalities, note further that

$$
\begin{aligned}
G_{21-1}(\tau) & \leq C \frac{\tau^{\frac{2}{3}}}{\left(1+\tau^{\frac{2}{3}}\right)^{2}} \int_{\delta^{3} \tau}^{2 \tau} \frac{(1+|\tau-\zeta|)^{2 b}}{(1+|\zeta|)^{\frac{2 s}{3}}} \frac{1}{3 \eta^{2}(\zeta)-1} d \zeta \\
& \leq C \frac{\tau^{\frac{2}{3}}(1+\tau)^{2 b}}{\left(1+\tau^{\frac{2}{3}}\right)^{2}} \int_{\delta^{3} \tau}^{2 \tau} \frac{1}{(1+\zeta)^{\frac{2+2 s}{3}}} d \zeta \\
& \leq C \tau^{\frac{6 b-2 s-1}{3}}
\end{aligned}
$$

and

$$
\begin{aligned}
G_{21-2}(\tau) & \leq C \tau^{\frac{2}{3}} \int_{2 \tau}^{\infty} \frac{(1+|\tau-\zeta|)^{2 b}}{\left(1+\tau^{\frac{2}{3}}+\zeta^{\frac{2}{3}}\right)^{2}(1+\zeta)^{\frac{2 s}{3}} \zeta^{\frac{2}{3}}} d \zeta \\
& \leq C \tau^{\frac{2}{3}} \int_{2 \tau}^{\infty} \frac{\zeta^{2 b}}{\zeta^{2\left(1+\frac{s}{3}\right)}} d \zeta \\
& \leq C \tau^{\frac{6 b-2 s-1}{3}}
\end{aligned}
$$

if $b<\frac{1}{2}+\frac{s}{3}$. The claim is thereby established. As a consequence, the following inequality emerges. For given $s \geq 0$ and $b<\frac{1}{2}+\frac{s}{3}$, there exists a constant $C$ such that

$$
\begin{aligned}
& \int_{\frac{2}{3 \sqrt{3}}}^{\infty} \phi_{2}(\mu(\tau))\left|\int_{0}^{\infty} h(s) e^{-i w \tau} d w\right|^{2} B_{21}(\tau) d \tau \\
& \quad \leq C \int_{\frac{2}{3 \sqrt{3}}}^{\infty} \phi_{2}(\mu(\tau)) \tau^{\frac{2(3 b-s-1 / 2)}{3}}\left|\int_{0}^{\infty} h(w) e^{-i w \tau} d w\right|^{2} d \tau \\
& \leq C\|h\|_{H^{(3 b-s-1 / 2) / 3}\left(R^{+}\right)}^{2}
\end{aligned}
$$

for any $h \in H^{\frac{3 b-s-1 / 2}{3}}\left(R^{+}\right)$. 
Next, consider the term

$$
A_{41}(\xi, \tau)=K_{41}(\xi, \tau) \phi_{2}(\mu(\tau)) \hat{h}(\tau)
$$

involving $K_{41}$. For given $b>0$ and $s \in R$,

$$
\begin{aligned}
& \int_{-\infty}^{\infty} \int_{0}^{\infty}\left(1+\left|\tau-\left(\xi^{3}-\xi\right)\right|\right)^{2 b}(1+|\xi|)^{-2 s}\left|A_{41}(\xi, \tau)\right|^{2}(1+\omega(\tau))^{2} \\
& (1-\Theta(\xi, \tau))^{2} d \xi d \tau \leq C \int_{\frac{2}{3 \sqrt{3}}}^{\infty} \phi_{2}(\mu(\tau))\left|\int_{0}^{\infty} e^{-i s \tau} h(s) d s\right|^{2} B_{41}(\tau) d \tau
\end{aligned}
$$

where $B_{41}(\tau)$ is equal to

$$
\int_{0}^{\infty} \frac{\left(4 \xi^{2}-\mu^{2}(\tau)\right)^{2} \mu(\tau)^{2}\left(1+\left|\tau-\left(\xi^{3}-\xi\right)\right|\right)^{2 b}(1+|\xi|)^{-2 s}(1+\omega(\tau))^{2}(1-\Theta(\xi, \tau))^{2}}{\left(3 \mu^{2}(\tau)-4+(2 \xi+\mu(\tau))^{2}\right)^{2}\left(3 \mu^{2}(\tau)-4+(2 \xi-\mu(\tau))^{2}\right)^{2}} d \xi
$$

As in the estimation of $B_{21}(\tau)$, one shows that if $b<\frac{1}{2}+\frac{s}{3}$,

$$
B_{41}(\tau)=O\left(\tau^{\frac{6 b-2 s-1}{3}}\right)
$$

as $\tau \rightarrow \infty$ using again the fact that $\mu(\tau) \sim \tau^{\frac{1}{3}}$ for large positive value of $\tau$. Consequently, for $0 \leq b<\frac{1}{2}+\frac{s}{3}$ and $s \geq 0$, there exists a constant $C$ such that

$$
\int_{\frac{2}{3 \sqrt{3}}}^{\infty} \phi_{2}(\mu(\tau))\left|\int_{0}^{\infty} h(s) e^{-i s \tau} d s\right|^{2} B_{41}(\tau) d \tau \leq C\|h\|_{H}^{2} \frac{3 b-s-1 / 2}{3}_{\left(R^{+}\right)} .
$$

The proof of Proposition 3.2 is complete.

Next, attention is given to the term

$$
\int_{-\infty}^{\infty} \int_{-\infty}^{\infty}\left(1+\left|\tau-\left(\xi^{3}-\xi\right)\right|\right)^{2 b}(1+|\xi|)^{-2 s}\left|\hat{I}_{22}(\xi, \tau)\right|^{2} d \xi d \tau
$$

for which we have the following estimate.

Proposition 3.3. Let $s$ and $b$ be given satisfying $0 \leq b<\frac{5}{6}+\frac{s}{3}<\frac{4}{3}$. Then there exists a constant $C$ such that

$$
\int_{-\infty}^{\infty} \int_{-\infty}^{\infty}\left(1+\left|\tau-\left(\xi^{3}-\xi\right)\right|\right)^{2 b}(1+|\xi|)^{-2 s}\left|\hat{I}_{22}(\xi, \tau)\right|^{2} d \xi d \tau<C\|h\|_{H}^{2}{ }_{{ }^{\frac{3 b-s-\frac{1}{2}}{3}}}\left(R^{+}\right)
$$

for any $h \in H^{\frac{3 b-s-\frac{1}{2}}{3}}\left(R^{+}\right)$.

Proof of Proposition 3.3: First, we note that to study $\hat{I}_{22}(\xi, \tau)$, we can use the form of $\hat{I}_{22}(\xi, \tau)$ in (2.15) or (2.24). As before, details are given for only one term in $\mathcal{F}_{t}\left[\int_{0}^{\infty} E(x, t) \cos (x \eta) d x\right]$, say

$$
A_{11}(\xi, \tau)=K_{11}(\xi, \tau) \phi_{2}(\mu(\tau)) \hat{h}(\tau) .
$$

Notice that $A_{11}(-\xi, \tau)=A_{21}(\xi, \tau)$. Hence, we may consider only the case wherein $\xi \geq 0$. Denote by $q_{2}$ the function

$$
q_{2}(\xi, \tau)=\frac{1}{3 \mu^{2}(\tau)-4+(2 \xi+\mu(\tau))^{2}}
$$


and, for $\xi \geq 1$, let $D_{2}$ be given by

$D_{2}(\xi, \tau)=2 \int_{0}^{\infty} \frac{\eta^{2} \Theta(\eta, \tau)}{\xi\left(\xi^{2}-\eta^{2}\right)} q_{2}(\eta, \tau) d \eta+(1+\omega(\tau)) \int_{0}^{\infty} \frac{\eta^{2}(1-\Theta(\eta, \tau))}{\xi\left(\xi^{2}-\eta^{2}\right)} q_{2}(\eta, \tau) d \eta$

for $\xi \geq \delta_{1} \mu(\tau)$ (from (2.24)) and

$$
D_{2}(\xi, \tau)=2 \int_{0}^{\infty} \frac{\xi \Theta(\eta, \tau)}{\left(\xi^{2}-\eta^{2}\right)} q_{2}(\eta, \tau) d \eta+(1+\omega(\tau)) \int_{0}^{\infty} \frac{\xi(1-\Theta(\eta, \tau))}{\left(\xi^{2}-\eta^{2}\right)} q_{2}(\eta, \tau) d \eta
$$

for $0 \leq \xi \leq \delta_{1} \mu(\tau)$ (from (2.15)), where $\delta_{1}>0$ is a small constant. The relevance of these functions will become clear presently. First, note that

$$
A_{11}(\xi, \tau)=q_{2}(\xi, \tau) \phi_{2}(\mu(\tau)) \hat{h}(\tau) \sqrt{3 \mu^{2}(\tau)-4} .
$$

As for $D_{2}$, changing variables in the integrals of its definition shows it to have the form

$$
\begin{aligned}
D_{2}(\xi, \tau)= & 2 \int_{0}^{\infty} \frac{\eta^{2}}{\xi\left(\xi^{2}-\eta^{2}\right)} \Theta(\eta, \tau) q_{2}(\eta, \tau) d \eta \\
& \quad+(1+\omega(\tau)) \int_{0}^{\infty} \frac{\eta^{2}}{\xi\left(\xi^{2}-\eta^{2}\right)}(1-\Theta(\eta, \tau)) q_{2}(\eta, \tau) d \eta \\
= & \frac{2}{\mu^{2}(\tau)} \int_{0}^{a_{0}} \frac{\eta^{2}}{y\left(y^{2}-\eta^{2}\right)} \Theta(\mu(\tau) \eta, \tau) p_{2}(\eta, \tau) d \eta \\
& \quad+\frac{1+\omega(\tau)}{\mu^{2}(\tau)} \int_{a_{1}}^{\infty} \frac{\eta^{2}}{y\left(y^{2}-\eta^{2}\right)}(1-\Theta(\mu(\tau) \eta, \tau)) p_{2}(\eta, \tau) d \eta \\
& :=D_{21}(y, \tau)+D_{22}(y, \tau)
\end{aligned}
$$

where

$a_{0}=\frac{\delta|\tau|^{\frac{1}{3}}+1}{\mu(\tau)}, \quad a_{1}=\frac{\delta|\tau|^{\frac{1}{3}}}{\mu(\tau)}, \quad y=\xi / \mu(\tau) \geq \delta_{1}, \quad p_{2}(\eta, \tau)=\frac{1}{2-\frac{4}{\mu^{2}(\tau)}+(2 \eta+1)^{2}}$.

We have similar definitions for $0 \leq y \leq \delta_{1}$. Remark that $a_{0}$ is bounded independently of $\tau$ and so for $y$ large enough, $y^{2}-\eta^{2}$ is bounded below for $\eta \in\left[0, a_{0}\right]$. Thus,

$$
\begin{aligned}
D_{21}(y, \tau) & =\frac{2}{y^{3} \mu^{2}(\tau)} \int_{0}^{a_{0}} \frac{\eta}{1-(\eta / y)^{2}} \Theta(\mu(\tau) \eta, \tau) p_{2}(\eta, \tau) d \eta \\
& :=\frac{1}{y^{3} \mu^{2}(\tau)} D_{21,2}(\tau, y)
\end{aligned}
$$

where

$$
\left|D_{21,2}(\tau, y)\right| \leq C \quad \text { for all } \tau \text { and } y \text { large. }
$$


Turning to $D_{22}$, note that $\Theta(\mu(\tau) \eta, \tau)=0$ for $\eta \geq a_{1}$, so

$$
\begin{aligned}
& \int_{a_{1}}^{\infty} \frac{\eta^{2}}{y\left(y^{2}-\eta^{2}\right)}(1-\Theta(\mu(\tau) \eta, \tau)) p_{2}(\eta, \tau) d \eta \\
& =\int_{a_{1}}^{\infty} \frac{\eta^{2}}{y\left(y^{2}-\eta^{2}\right)} p_{2}(\eta, \tau) d \eta \\
& =\frac{1}{y^{2}} \int_{\frac{a_{1}}{y}}^{\infty} \frac{1}{1-z^{2}} z^{2} y^{2} p_{2}(z y, \tau) d z \\
& =\frac{1}{y^{2}} \int_{\frac{a_{1}}{y}}^{\infty} \frac{1}{1-z^{2}}\left(z^{2} y^{2} p_{2}(z y, \tau)-1 / 4\right) d z+\frac{1}{4 y^{2}} \int_{\frac{a_{1}}{y}}^{\infty} \frac{1}{1-z^{2}} d z \\
& :=\frac{1}{y^{2}}\left(D_{22,1}(y, \tau)+D_{22,2}(y, \tau)\right) .
\end{aligned}
$$

Of course,

$$
D_{22,2}(y, \tau)=\frac{1}{4} \int_{\frac{a_{1}}{y}}^{\infty} \frac{1}{1-z^{2}} d z=-\frac{1}{4} \int_{0}^{\frac{a_{1}}{y}} \frac{1}{1-z^{2}} d z
$$

since

$$
\int_{0}^{\infty} \frac{1}{1-z^{2}} d z=0
$$

as a principal-value integral. It is therefore clear that

$$
\left|D_{22,2}(y, \tau)\right| \leq \frac{C}{y}
$$

for some constant $C$ independent of $\tau$ when $y$ is large, say $y \geq y_{0}$. As for $D_{22,1}(y, \tau)$, note that

$$
\begin{aligned}
\eta^{2} y^{2} p_{2}(\eta y, \tau)-1 / 4 & =\frac{1}{y}\left(\frac{4}{\mu^{2}(\tau) y}-\frac{3}{y}-8 \eta\right)\left(\frac{2}{y^{2}}-\frac{4}{\mu^{2}(\tau) y^{2}}+\left(2 \eta+\frac{1}{y}\right)^{2}\right)^{-1} \\
& :=\frac{1}{y} p^{*}(\eta, y, \tau) .
\end{aligned}
$$

Rewrite $D_{22,1}(y, \tau)$ as

$$
D_{22,1}(y, \tau)=\frac{1}{y}\left(\int_{a_{1} / y}^{1 / 2}+\int_{1 / 2}^{2}+\int_{2}^{\infty}\right) \frac{p^{*}(\eta, y, \tau)}{1-\eta^{2}} d \eta
$$

to obtain

$$
\left|\left(\int_{1 / 2}^{2}+\int_{2}^{\infty}\right) \frac{p^{*}(\eta, y, \tau)}{1-\eta^{2}} d \eta\right| \leq C
$$

and

$$
\left|\int_{a_{1} / y}^{1 / 2} \frac{p^{*}(\eta, y, \tau)}{1-\eta^{2}} d \eta\right| \leq C(1+\ln y)
$$

where $C$ is independent of $\tau$ and $y$ for $\mu(\tau) \geq 3$ and $y$ large. Thus, if $y \geq y_{0}$, then

$$
\left|D_{2}(\mu(\tau) y, \tau)\right| \leq \frac{C}{y^{3}}(1+\ln y) \frac{1}{\mu^{2}(\tau)}
$$


where $C$ is independent of $\tau$ and $y$. The following calculation shows the relevance of $D_{2}$;

$$
\begin{aligned}
& \int_{0}^{\infty} \int_{0}^{\infty}\left(1+\left|\tau-\left(\xi^{3}-\xi\right)\right|\right)^{2 b}(1+|\xi|)^{-2 s}\left|\int_{-\infty}^{\infty} \frac{1}{\xi-\eta} A_{11}(\eta, \tau) \Theta_{1}(\eta, \tau) d \eta\right|^{2} d \xi d \tau \\
& =\int_{0}^{\infty} \frac{1}{\pi^{2}} \phi_{2}^{2}(\mu(\tau))|\hat{h}|^{2}(\tau)\left(3 \mu^{2}(\tau)-4\right) \int_{0}^{\infty}\left(1+\left|\tau-\left(\xi^{3}-\xi\right)\right|\right)^{2 b}(1+|\xi|)^{-2 s} \\
& \quad \times\left|\int_{-\infty}^{\infty} \frac{1}{\xi-\eta} q_{2}(\eta, \tau) \Theta_{1}(\eta, \tau) d \eta\right|^{2} d \xi d \tau \\
& =\int_{0}^{\infty} \frac{1}{\pi^{2}} \phi_{2}^{2}(\mu(\tau))|\hat{h}|^{2}(\tau)\left(3 \mu^{2}(\tau)-4\right) \\
& \quad \times \int_{0}^{\infty}\left(1+\left|\tau-\left(\xi^{3}-\xi\right)\right|\right)^{2 b}(1+|\xi|)^{-2 s}\left|D_{2}(\xi, \tau)\right|^{2} d \xi d \tau .
\end{aligned}
$$

Appropriate bounds on $D_{2}$ yield bounds on the left-hand side of the last formula. Consider the quantity

$$
\begin{aligned}
E_{2}(\tau):= & \left(3 \mu^{2}(\tau)-4\right) \int_{0}^{\infty}\left(1+\left|\tau-\left(\xi^{3}-\xi\right)\right|\right)^{2 b}(1+|\xi|)^{-2 s}\left|D_{2}(\xi, \tau)\right|^{2} d \xi \\
= & \left(3 \mu^{2}(\tau)-4\right)\left(\int_{0}^{\delta_{1} \mu(\tau)}+\int_{\delta_{1} \mu(\tau)}^{y_{0} \mu(\tau)}+\int_{y_{0} \mu(\tau)}^{\infty}\right)\left(1+\left|\tau-\left(\xi^{3}-\xi\right)\right|\right)^{2 b} \\
& \quad \times(1+|\xi|)^{-2 s} D_{2}^{2}(\xi, \tau) d \xi \\
& :=E_{21}(\tau)+E_{22}(\tau)+E_{23}(\tau)
\end{aligned}
$$

where $\delta_{1}$ is again a small constant. By the choice of $\omega(\tau)$, it transpires that for large $\tau$,

$$
\begin{aligned}
& \left|E_{23}(\tau)\right| \leq C \tau^{\frac{2}{3}}(\mu(\tau))^{2} \int_{y_{0} \mu(\tau)}^{\infty} \xi^{6 b-2 s-6} d \xi \\
& \leq C \tau^{\frac{2}{3}} \mu(\tau)^{6 b-2 s-3} \int_{y_{0}}^{\infty} \xi^{6 b-2 s-6} d \xi \\
& \leq C \tau^{\frac{6 b-2 s-1}{3}}
\end{aligned}
$$

if $6 b-2 s-6<-1$, which is to say $b<\frac{s}{3}+\frac{5}{6}$. For $\delta_{1} \leq y \leq y_{0}$, say,

$$
\begin{aligned}
\left|D_{2}\right| \leq & \frac{C}{\mu^{2}(\tau)}\left(1+\mid \int_{0}^{a_{0}} \frac{1}{y-\eta} \Theta(\mu(\tau) \eta, \tau) p_{2}(\eta, \tau) d \eta\right. \\
& \left.+(1+\omega(\tau)) \int_{a_{1}}^{2 y_{0}} \frac{1}{y-\eta}(1-\Theta(\mu(\tau) \eta, \tau)) p_{2}(\eta, \tau) d \eta \mid\right) .
\end{aligned}
$$


Note that if $\delta_{1} \leq y \leq a_{0}$, then

$$
\begin{gathered}
\left|\int_{0}^{a_{0}} \frac{1}{y-\eta} \Theta(\mu(\tau) \eta, \tau) p_{2}(\eta, \tau) d \eta\right| \leq\left|\int_{0}^{a_{0}} \frac{\Theta(\mu(\tau) \eta, \tau)}{y-\eta}\left(p_{2}(\eta, \tau)-p_{2}(y, \tau)\right) d \eta\right| \\
+\left|p_{2}(y, \tau) \int_{0}^{a_{0}} \frac{\Theta(\mu(\tau) \eta, \tau)}{y-\eta} d \eta\right| \leq C .
\end{gathered}
$$

The same bound is valid if $a_{1} \leq y \leq y_{0}$. Thus,

$$
\left|D_{2}\right| \leq \frac{C}{\mu^{2}(\tau)}
$$

and

$$
\begin{aligned}
& \left|E_{22}(\tau)\right| \leq C \tau^{-\frac{2}{3}} \int_{\delta_{1} \mu(\tau)}^{y_{0} \mu(\tau)}\left(1+\left|\tau-\left(\xi^{3}-\xi\right)\right|\right)^{2 b}(1+|\xi|)^{-2 s} d \xi \\
& \leq C \tau^{-\frac{2}{3}}\left(\int_{\delta_{1} \mu(\tau)}^{\frac{1}{2} \tau^{\frac{1}{3}}}+\int_{\frac{1}{2} \tau^{\frac{1}{3}}}^{2 \tau^{\frac{1}{3}}}+\int_{2 \tau^{\frac{1}{3}}}^{y_{0} \mu(\tau)}\right)\left(1+\left|\tau-\left(\xi^{3}-\xi\right)\right|\right)^{2 b}(1+|\xi|)^{-2 s} d \xi \\
& \leq C \tau^{-\frac{2}{3}}\left(\tau^{2 b} \int_{\delta_{1} \mu(\tau)}^{\frac{1}{2} \tau^{\frac{1}{3}}}(1+|\xi|)^{-2 s} d \xi+\int_{\frac{1}{2} \tau^{\frac{1}{3}}}^{2 \tau^{\frac{1}{3}}}\left(1+\left|\tau-\left(\xi^{3}-\xi\right)\right|\right)^{2 b} \xi^{-2 s} d \xi\right. \\
& \left.\quad+\int_{2 \tau^{\frac{1}{3}}}^{y_{0} \mu(\tau)} \xi^{6 b-2 s} d \xi\right) \\
& \leq C \tau^{-\frac{2}{3}}\left(\tau^{2 b+\frac{1-2 s}{3}}+\int_{\frac{1}{2}}^{2}(1+\tau|\xi-1|)^{2 b} \tau^{\frac{1-2 s}{3}} \xi d \xi+\tau^{\frac{6 b-2 s+1}{3}}\right) \\
& \leq C \tau^{-\frac{2}{3}}\left(\tau^{\frac{6 b-2 s+1}{3}}+\tau^{2 b+\frac{1-2 s}{3}}\right) \\
& \leq C \tau^{\frac{6 b-2 s-1}{3}}
\end{aligned}
$$

since $b \geq 0$. If $0 \leq y=\frac{\xi}{\mu(\tau)} \leq \delta_{1}$ in the term $D_{2}=D_{21}+D_{22}$, then

$$
\left|D_{22}\right| \leq \frac{C}{\mu^{2}(\tau)}\left|\int_{a_{1}}^{\infty} \frac{2 y}{y^{2}-\eta^{2}}(1-\Theta(\mu(\tau) \eta, \tau)) p_{2}(\eta, \tau) d \eta\right| \leq C \frac{|y|}{\mu^{2}(\tau)}
$$

and

$$
\begin{aligned}
& D_{21}=\frac{1}{\mu^{2}(\tau)}\left[\int_{0}^{a_{0}} \frac{1}{y-\eta}\left(p_{2}(\eta, \tau)-p_{2}(y, \tau)\right) \Theta(\mu(\tau) \eta, \tau) d \eta\right. \\
& +\int_{0}^{a_{0}} \frac{1}{y+\eta}\left(p_{2}(\eta, \tau)-p_{2}(-y, \tau)\right) \Theta(\mu(\tau) \eta, \tau) d \eta \\
& \left.+\int_{0}^{a_{0}}\left(\frac{1}{y-\eta} p_{2}(y, \tau)+\frac{1}{y+\eta} p_{2}(-y, \tau)\right) \Theta(\mu(\tau) \eta, \tau) d \eta\right] \\
& :=\frac{1}{\mu^{2}(\tau)}\left(D_{21-1}+D_{21-2}+D_{21-3}\right) \text {. }
\end{aligned}
$$


Recall that $p_{2}(\eta, \tau)=\left(3-\frac{4}{\mu^{2}(\tau)}+(2 \eta+1)^{2}\right)^{-1}$, so that

$$
D_{21-1}(y, \tau)+D_{21-2}(y, \tau)=
$$

$$
\begin{gathered}
=\int_{0}^{a_{0}} \frac{\Theta(\mu(\tau) \eta, \tau)}{3-\frac{4}{\mu^{2}(\tau)}+(2 \eta+1)^{2}}\left(\frac{\eta+y+1}{3-\frac{4}{\mu^{2}(\tau)}+(2 y+1)^{2}}-\frac{\eta-y+1}{3-\frac{4}{\mu^{2}(\tau)}+(-2 y+1)^{2}}\right) d \eta \\
=\int_{0}^{a_{0}} \frac{\Theta(\mu(\tau) \eta, \tau)}{3-\frac{4}{\mu^{2}(\tau)}+(2 \eta+1)^{2}}\left[\frac{-8 y(\eta+1)}{\left(3-\frac{4}{\mu^{2}(\tau)}+(2 y+1)^{2}\right)\left(3-\frac{4}{\mu^{2}(\tau)}+(-2 y+1)^{2}\right)}\right. \\
\left.\quad+y\left(\frac{1}{3-\frac{4}{\mu^{2}(\tau)}+(2 y+1)^{2}}+\frac{1}{3-\frac{4}{\mu^{2}(\tau)}+(-2 y+1)^{2}}\right)\right] d \eta .
\end{gathered}
$$

It thus transpires that

$$
\left|D_{21-1}+D_{21-2}\right| \leq C|y|
$$

Also,

$$
\begin{aligned}
& D_{21-3}=p_{2}(y, \tau) \int_{0}^{a_{0}} \frac{1}{y-\eta} \Theta(\mu(\tau) \eta, \tau) d \eta+p_{2}(-y, \tau) \int_{0}^{a_{0}} \frac{1}{y+\eta} \Theta(\mu(\tau) \eta, \tau) d \eta \\
& =p_{2}(y, \tau)\left(\int_{0}^{a_{1}} \frac{1}{y-\eta} d \eta+\int_{a_{1}}^{a_{0}} \frac{1}{y-\eta} \Theta(\mu(\tau) \eta, \tau) d \eta\right) \\
& +p_{2}(-y, \tau)\left(\int_{0}^{a_{1}} \frac{1}{y+\eta} d \eta+\int_{a_{1}}^{a_{0}} \frac{1}{y+\eta} \Theta(\mu(\tau) \eta, \tau) d \eta\right) \\
& =p_{2}(y, \tau)\left(-\ln \left|a_{1}-y\right|+\ln |y|\right)+p_{2}(-y, \tau)\left(\ln \left|a_{1}+y\right|-\ln |y|\right) \\
& +p_{2}(y, \tau) \int_{a_{1}}^{a_{0}} \frac{1}{\eta} \frac{\Theta(\mu(\tau) \eta, \tau) d \eta}{\frac{y}{\eta}-1}+p_{2}(-y, \tau) \int_{a_{1}}^{a_{0}} \frac{1}{\eta} \frac{\Theta(\mu(\tau) \eta, \tau) d \eta}{\frac{y}{\eta}+1} \\
& =\left(-p_{2}(y, \tau)+p_{2}(-y, \tau)\right)\left(\ln \left|a_{1}\right|-\ln |y|\right)+p_{2}(y, \tau)\left(-\ln \left|1-\frac{y}{a_{1}}\right|\right) \\
& +p_{2}(-y, \tau) \ln \left(1+\frac{y}{a_{1}}\right)+p_{2}(y, \tau) \int_{a_{1}}^{a_{0}} \frac{1}{\eta}\left(-1+\frac{y / \eta}{y / \eta-1}\right) \Theta(\mu(\tau) \eta, \tau) d \eta \\
& +p_{2}(-y, \tau) \int_{a_{1}}^{a_{0}} \frac{1}{\eta}\left(1-\frac{y / \eta}{y / \eta+1}\right) \Theta(\mu(\tau) \eta, \tau) d \eta \\
& =\left(-p_{2}(y, \tau)+p_{2}(-y, \tau)\right)\left(\ln \left|a_{1}\right|-\ln |y|+\int_{a_{1}}^{a_{0}} \frac{1}{\eta} \Theta(\mu(\tau) \eta, \tau) d \eta\right) \\
& +p_{2}(y, \tau)\left(-\ln \left(1-\frac{y}{a_{1}}\right)+\int_{a_{1}}^{a_{0}} \frac{y}{(y-\eta) \eta} \Theta(\mu(\tau) \eta, \tau) d \eta\right) \\
& +p_{2}(-y, \tau)\left(\ln \left(1+\frac{y}{a_{1}}\right)-\int_{a_{1}}^{a_{0}} \frac{y}{(y+\eta) \eta} \Theta(\mu(\tau) \eta, \tau) d \eta\right) .
\end{aligned}
$$

It follows that

$$
\left|D_{21-3}\right| \leq C|y|(|\ln | y||+1)
$$


and

$$
\left|D_{21}\right| \leq \frac{C|y|(|\ln | y||+1)}{\mu^{2}(\tau)},
$$

which implies that

$$
\left|D_{2}\right| \leq \frac{C|y|(|\ln | y||+1)}{\mu^{2}(\tau)} .
$$

Thus, it is apparent that

$$
\begin{aligned}
& \left|E_{21}\right| \leq C \tau^{2 / 3} \int_{0}^{\delta_{1} \mu(\tau)}(1+|\tau|)^{2 b}(1+|\xi|)^{-2 s} \frac{\xi^{2}}{\tau^{2}}\left(1+|\ln | \frac{\xi}{\mu(\tau)} \mid\right) d \xi \\
& \leq C \tau^{-4 / 3}(1+|\tau|)^{2 b} \int_{0}^{\delta_{1} \mu(\tau)}(1+|\xi|)^{-2 s}\left(1+|\ln | \frac{\xi}{\mu(\tau)}||\right) \xi^{2} d \xi \\
& \leq C \tau^{-4 / 3}(1+|\tau|)^{2 b} \int_{0}^{\delta_{1}}(1+|\mu(\tau)||\xi|)^{-2 s} \mu^{3}(\tau)(1+|\ln | \xi||) \xi^{2} d \xi \\
& \leq C \tau^{-\frac{1}{3}+2 b-\frac{2 s}{3}} \int_{0}^{\delta_{1}} \xi^{2-2 s}(1+|\ln | \xi||) d \xi \\
& \leq C \tau^{2 b-(2 s+1) / 3}
\end{aligned}
$$

if $2-2 s>-1$. Combining these estimates, there obtains

$$
\left|E_{2}(\tau)\right| \leq C \tau^{2 b-(2 s+1) / 3}
$$

if $s<\frac{3}{2}$ and $0<b<\frac{1}{2}+\frac{s}{3}$. This in turn implies that

$$
\begin{aligned}
& \int_{-\infty}^{\infty} \int_{0}^{\infty}\left(1+\left|\tau-\left(\xi^{3}-\xi\right)\right|\right)^{2 b}(1+|\xi|)^{-2 s} \mid \int_{-\infty}^{\infty} \frac{1}{\xi-\eta} A_{21}(\eta, \tau) \\
& \times\left.(2 \Theta(\eta, \tau)+(1-\Theta(\eta, \tau))(1+\omega(\tau))) d \eta\right|^{2} d \xi d \tau \\
& \leq C \int_{0}^{\infty} \tau^{2 b-(2 s+1) / 3}\left|\int_{0}^{\infty} h(w) e^{-i w \tau} d w\right|^{2} d \tau \leq C\|h\|_{H^{b-\frac{s}{3}-\frac{1}{6}}}^{2}
\end{aligned}
$$

Similar estimates for the other terms yield, in sum,

$$
\int_{-\infty}^{\infty} \int_{-\infty}^{\infty}\left(1+\left|\tau-\left(\xi^{3}-\xi\right)\right|\right)^{2 b}(1+|\xi|)^{-2 s}\left|\hat{I}_{22}(\xi, \tau)\right|^{2} d \xi d \tau \leq C\|h\|_{H^{b-\frac{s}{3}-\frac{1}{6}}}^{2}
$$

if $0 \leq s<3 / 2$ and $0 \leq b<\frac{5}{6}+\frac{s}{3}$. This completes the proof of the Proposition 3.3. $\square$.

By combining above two propositions, we obtain the estimate (3.3). The proof of Theorem 3.1 is complete.

Now, attention is turned to the operator $\mathcal{B} I_{m 2}(t)$. 
THEOREM 3.4. Let $\psi(t)$ be a given smooth function of $t$ with compact support and assume that $0 \leq b<\frac{5}{6}+\frac{s}{3}$ with $-1 \leq s<\frac{1}{2}$. Then there exists a constant $C$ such that

$$
\Lambda_{-s, b}\left(\psi \mathcal{B} I_{m 2}(h)\right) \leq C\|h\|_{H^{\frac{3 b-s-1 / 2}{3}}\left(R^{+}\right)}
$$

for any $h \in H_{0}^{\frac{3 b-s-1 / 2}{3}}\left(R^{+}\right)$.

Proof: As in the proof of Theorem 3.1, it suffices to prove the following two propositions.

Proposition 3.5. Let $s \in R$ and $b \geq 0$ be given. There exists a constant $C$ such that

$$
\begin{aligned}
& \int_{-\infty}^{\infty} \int_{-\infty}^{\infty}\left(1+\left|\tau-\left(\xi^{3}-\xi\right)\right|\right)^{2 b}(1+|\xi|)^{-2 s}\left|\hat{I}_{21}^{*}(\xi, \tau)\right|^{2} d \xi d \tau \\
& \quad \leq\left\{\begin{array}{cc}
C\|h\|^{2} H^{\frac{9 b-s-\frac{5}{2}}{9}} & \text { if } s \leq \frac{1}{2} \\
C\|h\|_{H^{\frac{3 b-1}{3}}}^{2} & \text { if } s \geq \frac{1}{2} .
\end{array}\right.
\end{aligned}
$$

Proposition 3.6. Let $s$ and $b$ be given satisfying $0 \leq b<\frac{5}{6}+\frac{s}{3}$. There exists a constant $C$ such that

$$
\int_{-\infty}^{\infty} \int_{-\infty}^{\infty}\left(1+\left|\tau-\left(\xi^{3}-\xi\right)\right|\right)^{2 b}(1+|\xi|)^{-2 s}\left|\hat{I}_{22}^{*}(\xi, \tau)\right|^{2} d \xi d \tau \leq C\|h\|_{H}^{2}{ }_{\underbrace{\frac{3 b-s-\frac{1}{2}}{3}}\left(R^{+}\right)}
$$

for any $h \in H^{\frac{3 b-s-\frac{1}{2}}{3}}\left(R^{+}\right)$.

We only present a proof of Proposition 3.5. The proof of Proposition 3.6 follows the same line as that of Proposition 3.3 and is therefore omitted. As in the proof of Proposition 3.2, detailed analysis is given for the term containing $K_{21}$; the estimates for the other terms are sufficiently similar that their proof does not require further elaboration. Suppose $\xi \geq 1$ and $\tau \geq 0$ in what follows. The other cases are entirely analogous. Define

$$
A_{m 1}(\xi, \tau)=K_{m 1}(\xi, \tau) \phi_{2}(\mu(\tau)) \hat{h}(\tau), \quad m=1,2,3
$$

For given $s$ and $b \geq 0$, we have

$$
\begin{aligned}
& \int_{0}^{\infty} \int_{1}^{\infty}\left(1+\left|\tau-\left(\xi^{3}-\xi\right)\right|\right)^{2 b}(1+|\xi|)^{-2 s}\left|A_{21}(\xi, \tau)(1+\omega(\tau)) \Theta(\xi, \tau)\right|^{2} d \xi d \tau \\
& \quad \leq C \int_{-\infty}^{\infty} \phi_{2}(\mu(\tau))\left|\int_{0}^{\infty} h(s) e^{-i s \tau} d s\right|^{2} B_{21}^{*}(\tau) d \tau
\end{aligned}
$$


with

$$
\begin{aligned}
& B_{21}^{*}(\tau)=\int_{1}^{\infty}(1+\left.\left|\tau-\left(\xi^{3}-\xi\right)\right|\right)^{2 b}(1+|\xi|)^{-2 s}|(1+\omega(\tau)) \Theta(\xi, \tau)|^{2} \\
& \times \frac{\left(3 \mu^{2}(\tau)-4\right) \phi_{2}(\mu(\tau))}{\left(3 \mu^{2}(\tau)-4+(2 \xi-\mu(\tau))^{2}\right)^{2}} d \xi \\
& \leq C \int_{1}^{\delta \tau^{\frac{1}{3}}}\left(1+\left|\tau-\left(\xi^{3}-\xi\right)\right|\right)^{2 b}(1+|\xi|)^{-2 s} \frac{\left(3 \mu^{2}(\tau)-4\right) \phi_{2}(\mu(\tau))}{\left(3 \mu^{2}(\tau)-4+(2 \xi-\mu(\tau))^{2}\right)^{2}} d \xi
\end{aligned}
$$

because of the properties of $\Theta$, and where $\delta>0$ is, as before, fixed, but arbitrary for the moment. Let $\xi=\eta(\zeta)$ be the real solution of the equation

$$
\xi^{3}-\xi=\zeta, \quad 0 \leq \zeta<\infty, \quad \text { for which } \quad 1 \leq \xi<\infty
$$

Note that $\eta(\zeta) \sim \zeta^{\frac{1}{3}}$ as $\zeta \rightarrow \infty$. For large $\tau$, note also that $\mu(\tau) \sim \tau^{\frac{1}{3}}$. Thus, for $\tau>0$ large enough that $\delta \tau^{1 / 3}>1$,

$$
\begin{aligned}
B_{21}^{*}(\tau) & \leq C \int_{0}^{\delta \tau^{\frac{1}{3}}} \frac{\left(3 \mu^{2}(\tau)-4\right)(1+|\tau-\zeta|)^{2 b}}{\left(3 \mu^{2}(\tau)-4+(2 \eta(\zeta)-\mu(\tau))^{2}\right)^{2}}(1+|\zeta|)^{-\frac{2 s}{3}} \frac{1}{3 \eta^{2}(\zeta)-1} d \zeta \\
& \leq C \int_{0}^{\delta \tau^{\frac{1}{3}}} \frac{\tau^{\frac{2}{3}}(1+|\tau-\zeta|)^{2 b}}{\left(1+3 \tau^{\frac{2}{3}}+\left(2 \eta(\zeta)-\tau^{\frac{1}{3}}\right)^{2}\right)^{2}}(1+|\zeta|)^{-\frac{2 s}{3}} \frac{1}{3 \eta^{2}(\zeta)-1} d \zeta \\
& \leq C \frac{\tau^{\frac{2}{3}}}{\left(1+\tau^{\frac{2}{3}}\right)^{2}} \int_{0}^{\delta \tau^{\frac{1}{3}}} \frac{(1+|\tau-\zeta|)^{2 b}}{(1+|\zeta|)^{\frac{2 s}{3}}} \frac{1}{3 \eta^{2}(\zeta)-1} d \zeta \\
& \leq C \frac{\tau^{\frac{2}{3}}}{\left(1+\tau^{\left.\frac{2}{3}\right)^{2}} \int_{0}^{\delta \tau^{\frac{1}{3}}} \frac{(1+|\tau-\zeta|)^{2 b}}{(1+\zeta)^{\frac{2+2 s}{3}}} d \zeta\right.} \\
& \leq C \tau^{\frac{6 b-2}{3}\left(1+\tau^{\frac{1-2 s}{9}}\right)} \\
& \leq\left\{\begin{array}{l}
C \tau^{\frac{18 b-2 s-5}{9}} \quad \text { if } s \leq \frac{1}{2}, \\
C \tau^{\frac{6 b-2}{3}} s \geq \frac{1}{2} .
\end{array}\right.
\end{aligned}
$$


In consequence,

$$
\begin{aligned}
& \int_{0}^{\infty} \int_{0}^{\infty}\left(1+\left|\tau-\left(\xi^{3}-\xi\right)\right|\right)^{2 b}(1+|\xi|)^{-2 s}\left|A_{21}(\xi, \tau)(1+\omega(\tau)) \Theta(\xi, \tau)\right|^{2} d \xi d \tau \\
& \quad \leq C \int_{0}^{\infty} \phi_{2}(\mu(\tau))\left|\int_{0}^{\infty} h(s) e^{-i s \tau} d s\right|^{2} B_{21}^{*}(\tau) d \tau \\
& \quad \leq C \int_{0}^{\infty} \phi_{2}(\mu(\tau))\left|\int_{0}^{\infty} h(s) e^{-i s \tau} d s\right|^{2} \begin{cases}C \tau^{\frac{18 b-2 s-5}{9}} d \tau & \text { if } s \leq \frac{1}{2}, \\
C \tau^{\frac{6 b-2}{3}} d \tau & \text { if } s \geq \frac{1}{2} .\end{cases} \\
& \quad=\left\{\begin{array}{cl}
C\|h\|^{2} H^{\frac{9 b-s-\frac{5}{2}}{9}} & \text { if } s \leq \frac{1}{2}, \\
C\|h\|_{H^{\frac{3 b-1}{3}}}^{2} & \text { if } s \geq \frac{1}{2} .
\end{array}\right.
\end{aligned}
$$

The proof is complete.

Finally, consider the operator $\mathcal{B} I_{e}(t)$.

THEOREM 3.7. Let $\psi(t)$ be a given smooth function of $t$ with compact support. Assume that

$$
\begin{cases}0 \leq b<\frac{1}{2}+\frac{s}{3} & \text { if } s \geq 0, \\ 0 \leq b<\frac{1}{2} & \text { if }-1 \leq s<0 .\end{cases}
$$

Then there exists a constant $C$ such that

$$
\Lambda_{-s, b}\left(\psi \mathcal{B} I_{e} h\right) \leq \begin{cases}C\|h\|_{H} \frac{3 b-s-1 / 2}{3}\left(^{+}\right) & \text {if } s \leq \frac{1}{2}, \\ C\|h\|_{H} \frac{3 b-1}{3}\left(R^{+}\right) & \text {if } s \geq \frac{1}{2} .\end{cases}
$$

for any $h \in H_{0}^{\frac{3 b-s-1 / 2}{3}}\left(R^{+}\right)$if $s \leq \frac{1}{2}$ and for any $h \in H_{0}^{\frac{3 b-1}{3}}\left(R^{+}\right)$if $s \geq \frac{1}{2}$.

Proof: To establish (3.15) it suffices to prove the following proposition.

Proposition 3.8. Assume that

$$
\begin{cases}0 \leq b<\frac{1}{2}+\frac{s}{3} & \text { if } s \geq 0, \\ 0 \leq b<\frac{1}{2} & \text { if }-1 \leq s<0 .\end{cases}
$$

Then there exists a constant $C$ such that

$$
\Lambda_{-s, b}\left(\mathcal{F}_{x, t}\left[I_{2}\right]\right) \leq \begin{cases}C\|h\|_{H} \frac{3 b-s-1 / 2}{3}{ }_{\left(R^{+}\right)} & \text {if } s \leq \frac{1}{2}, \\ C\|h\|_{H}{ }_{\frac{3 b-1}{3}}{ }_{\left(R^{+}\right)} & \text {if } s \geq \frac{1}{2} .\end{cases}
$$

for any $h \in H_{0}^{\frac{3 b-s-1 / 2}{3}}\left(R^{+}\right)$if $s \leq \frac{1}{2}$ and for any $h \in H_{0}^{\frac{3 b-1}{3}}\left(R^{+}\right)$if $s \geq \frac{1}{2}$.

To see $(3.16)$ is true, write $\mathcal{F}_{x, t}\left[I_{2}\right](\eta, \tau)$ as

$$
\mathcal{F}_{x, t}\left[I_{2}\right](\eta, \tau)=\mathcal{F}_{x, t}\left[I_{2}\right](\eta, \tau) \Theta(\eta, \tau)+\mathcal{F}_{x, t}\left[I_{2}\right](\eta, \tau)(1-\Theta(\eta, \tau)) .
$$

Then, (3.16) is seen to follow from Proposition 3.2 and Proposition 3.5. 
Observe that

$$
\|w\|_{L_{2}\left(0, T ; H^{s}(R)\right)} \leq C \Lambda_{s, b}(\psi w)
$$

for any $s \in R$ and $b \geq 0$, where $\psi \in C_{0}^{\infty}(R)$ and $\psi(t)=1$ when $t \in(0, T)$. The following theorem, which follows from Theorem 3.1, reveals a boundary smoothing property of the linear KdV-equation.

TheOrem 3.9. For any $T>0$ and $s \geq-\frac{3}{2}$, there exists a constant $C$ such that

$$
\left\|\mathcal{W}_{b d r} h\right\|_{L_{2}\left(0, T ; H^{s+\frac{3}{2}}\left(R^{+}\right)\right)} \leq C\|h\|_{H^{\frac{1+s}{3}}\left(R^{+}\right)}
$$

for any $h \in H_{0}^{\frac{1+s}{3}}\left(R^{+}\right)$.

Proof: From Theorem 3.1, the inequality

$$
\left\|\mathcal{W}_{b d r} h\right\|_{L_{2}\left(0, T ; H^{s}\left(R^{+}\right)\right)} \leq \Lambda_{s, b}\left(\psi \mathcal{B} I_{m 1} h\right) \leq C\|h\|_{H} \frac{3 b+s-1 / 2}{3}{ }_{\left(R^{+}\right)}
$$

follows, for some constant $C$ and $0 \leq b<\frac{1}{2}+\frac{s}{3}$. Estimate (3.17) with $s=0$ is seen to be valid by taking $b=0$ and $s=\frac{3}{2}$. To show (3.17) holds for $s>0$, let $u=\mathcal{W}_{b d r} h$ and $w=u_{t}$. Then $w=\mathcal{W}_{b d r} h^{\prime}$. In addition, note that

$$
\left\|\mathcal{W}_{b d r} h\right\|_{L_{2}\left(0, T ; H^{3}\left(R^{+}\right)\right)} \leq C\|w\|_{L_{2}\left(0, T ; L^{2}\left(R^{+}\right)\right)} .
$$

Thus, we have

$$
\begin{aligned}
\left\|\mathcal{W}_{b d r} h\right\|_{L_{2}\left(0, T ; H^{3+(3 / 2)}\left(R^{+}\right)\right)} & \leq C\left\|\mathcal{W}_{b d r} h^{\prime}\right\|_{L_{2}\left(0, T ; H^{(3 / 2)}\left(R^{+}\right)\right)} \\
& \leq C\|h\|_{H^{\frac{4}{3}\left(R^{+}\right)}} .
\end{aligned}
$$

By interpolation, (3.17) holds for $0 \leq s \leq 3$. A similar argument shows that it holds for any $s>3$. The proof is complete.

Remark: It was proved in $[4]$ that $h \in H_{0}^{\frac{1+s}{3}}\left(R^{+}\right)$implies that

$$
\mathcal{W}_{b d r} h \in C\left([0, T] ; H^{s}\left(R^{+}\right)\right) \cap L^{2}\left(0, T ; H_{l o c}^{s+1}\left(R^{+}\right)\right) \text {. }
$$

Theorem 3.9 does not follow from this. It represents a new, somewhat more subtle smoothing property.

To conclude this section, we present another estimate of $\mathcal{B} I_{m 1} h$ and $\mathcal{B} I_{m 2} h$ in the space $C\left(R ; H^{s}(R)\right)$ which will be useful in studying the well-posedness problem for the IBVP (1.1) in the next section. The main difficulty in its proof is to discover how to efficiently convert spatial regularity of $\mathcal{B} I_{m j} h, j=1,2$ to temporal regularity of the boundary value function $h$.

LEMMA 3.10. For $-\frac{3}{2}<\alpha<\frac{1}{2}$, the inequality

$$
\sup _{t \in R}\left\|\mathcal{B} I_{m 1} h\right\|_{H^{\alpha}(R)} \leq C\|h\|_{H^{\frac{\alpha+1}{3}}\left(R^{+}\right)}
$$

holds for some constant $C$; for $-\frac{1}{2}<\alpha<1$, there is a constant $C$ such that

$$
\sup _{t \in R}\left\|\mathcal{B} I_{m 2} h\right\|_{H^{\alpha}(R)} \leq C\|h\|_{H^{\frac{\alpha+1}{3}}\left(R^{+}\right)} .
$$


Proof: We provide the proof of (3.18). The proof of (3.19) is very similar. According to the proof of Theorem 3.1, it suffices to show that

$$
\begin{aligned}
& \mathcal{P}_{21}(\xi, t)=\int_{-\infty}^{\infty} e^{i \lambda t} q_{21}(\xi, \lambda) \phi_{2}(\mu(\lambda)) \\
& \int_{0}^{\infty} h(s) e^{-i \lambda s} d s(1-\Theta(\xi, \lambda))(1+\nu(\xi) \omega(\lambda)) d \lambda \\
& +\frac{2 i}{\pi \xi} \int_{-\infty}^{\infty} e^{\lambda t i} \int_{0}^{\infty} \frac{\eta^{2}}{\xi^{2}-\eta^{2}} q_{21}(\eta, \lambda) \phi_{2}(\mu(\lambda)) \\
& \int_{0}^{\infty} h(s) e^{-i \lambda s} d s \Theta_{1}(\eta, \lambda) d \eta d \lambda \\
& =\mathcal{P}_{21-1}(\xi, t)+\mathcal{P}_{21-2}(\xi, t)
\end{aligned}
$$

satisfies

$$
\sup _{t \in R} \int_{0}^{\infty}(1+|\xi|)^{2 \alpha}\left|\mathcal{P}_{21-j}(\xi, t)\right|^{2} d \xi \leq C\|h\|_{H^{\frac{\alpha+1}{3}}}^{2}\left(R^{+}\right)
$$

for $j=1,2$, where

$$
q_{21}(\xi, \lambda)=\frac{\sqrt{3 \mu^{2}(\lambda)-4}}{3 \mu^{2}(\lambda)-4+(2 \xi-\mu(\lambda))^{2}} .
$$

Toward establishing these inequalities, we first study $\mathcal{P}_{21-1}$. Observe that

$$
\begin{aligned}
& \int_{0}^{\infty}(1+|\xi|)^{2 \alpha}\left|\mathcal{P}_{21-1}(\xi, t)\right|^{2} d \xi \\
& =\int_{0}^{\infty}(1+|\xi|)^{2 \alpha} \mid \int_{-\infty}^{\infty} e^{\lambda_{1} t i} q_{21}\left(\xi, \lambda_{1}\right) \phi_{2}\left(\mu\left(\lambda_{1}\right)\right) \hat{h}\left(\lambda_{1}\right) \\
& \left(1-\Theta\left(\xi, \lambda_{1}\right)\right)\left(1+\nu(\xi) \omega\left(\lambda_{1}\right)\right) d \lambda_{1} \mid \\
& \quad \times\left|\int_{-\infty}^{\infty} e^{\lambda_{2} t i} q_{21}\left(\xi, \lambda_{2}\right) \phi_{2}\left(\mu\left(\lambda_{2}\right)\right) \hat{h}\left(\lambda_{2}\right)\left(1-\Theta\left(\xi, \lambda_{2}\right)\right)\left(1+\nu(\xi) \omega\left(\lambda_{2}\right)\right) d \lambda_{2}\right| d \xi \\
& \leq \int_{-\infty}^{\infty} \int_{-\infty}^{\infty} \phi_{2}\left(\mu\left(\lambda_{2}\right)\right)\left|\hat{h}\left(\lambda_{2}\right)\right| \phi_{2}\left(\mu\left(\lambda_{1}\right)\right)\left|\hat{h}\left(\lambda_{1}\right)\right| \\
& \quad \times\left(\int_{0}^{\infty}(1+|\xi|)^{2 \alpha} q_{21}\left(\xi, \lambda_{1}\right) q_{21}\left(\xi, \lambda_{2}\right)\left(1-\Theta\left(\xi, \lambda_{1}\right)\right)\left(1-\Theta\left(\xi, \lambda_{2}\right)\right) d \xi\right) d \lambda_{1} d \lambda_{2} .
\end{aligned}
$$

Now, estimate the term

$$
I\left(\lambda_{1}, \lambda_{2}\right)=\int_{0}^{\infty}(1+|\xi|)^{2 \alpha} q_{21}\left(\xi, \lambda_{1}\right) q_{21}\left(\xi, \lambda_{2}\right)\left(1-\Theta\left(\xi, \lambda_{1}\right)\right)\left(1-\Theta\left(\xi, \lambda_{2}\right)\right) d \xi .
$$

Since $\mu(\lambda) \sim O\left(\lambda^{1 / 3}\right)$ as $\lambda \rightarrow \infty$,

$$
\left|I\left(\lambda_{1}, \lambda_{2}\right)\right| \leq C \lambda_{1}^{1 / 3} \lambda_{2}^{1 / 3} \int_{0}^{\infty} \frac{(1+|\xi|)^{2 \alpha}\left(1-\Theta\left(\xi, \lambda_{1}\right)\right)\left(1-\Theta\left(\xi, \lambda_{2}\right)\right) d \xi}{\left(1+\lambda_{1}^{2 / 3}+\left(2 \xi-\mu\left(\lambda_{1}\right)\right)^{2}\right)\left(1+\lambda_{2}^{2 / 3}+\left(2 \xi-\mu\left(\lambda_{2}\right)\right)^{2}\right)}
$$


By the symmetry of this expression in $\lambda_{1}$ and $\lambda_{2}$, it suffices to consider only the case $0 \leq \lambda_{1} \leq \lambda_{2}$. Fix $\delta>0$ and break up the integral $I I\left(\lambda_{1}, \lambda_{2}\right)$ as before;

$$
\begin{aligned}
& I I\left(\lambda_{1}, \lambda_{2}\right)=\int_{0}^{\infty} \frac{(1+|\xi|)^{2 \alpha}\left(1-\Theta\left(\xi, \lambda_{1}\right)\right)\left(1-\Theta\left(\xi, \lambda_{2}\right)\right) d \xi}{\left(1+\lambda_{1}^{2 / 3}+\left(2 \xi-\mu\left(\lambda_{1}\right)\right)^{2}\right)\left(1+\lambda_{2}^{2 / 3}+\left(2 \xi-\mu\left(\lambda_{2}\right)\right)^{2}\right)} \\
& =\left(\int_{\delta\left|\lambda_{2}\right|}^{\max \left(\delta\left|\lambda_{2}\right|,\left|\mu\left(\lambda_{1}\right)\right|\right)}+\int_{\max \left(\delta\left|\lambda_{2}\right|,\left|\mu\left(\lambda_{1}\right)\right|\right)}^{2\left|\mu\left(\lambda_{2}\right)\right|}+\int_{2\left|\mu\left(\lambda_{2}\right)\right|}^{\infty}\right)(\cdots) d \xi \\
& :=I I_{1}\left(\lambda_{1}, \lambda_{2}\right)+I I_{2}\left(\lambda_{1}, \lambda_{2}\right)+I I_{3}\left(\lambda_{1}, \lambda_{2}\right) .
\end{aligned}
$$

Note that $\left(2 \xi-\mu\left(\lambda_{1}\right)\right)^{2} \geq\left(2 \xi-\left|\mu\left(\lambda_{1}\right)\right|\right)^{2}$. In the integral $I I_{1}$, either the limits of integration are the same, so the integral is zero, or, for $\lambda_{1}$ and $\lambda_{2}$ large, $0<C_{0} \lambda_{2} \leq$ $\lambda_{1} \leq \lambda_{2}$ for some $C_{0}>0$. In this case,

$$
\begin{aligned}
& I I_{1}\left(\lambda_{1}, \lambda_{2}\right) \leq C \int_{\delta\left|\lambda_{1}\right|}^{\left|\mu\left(\lambda_{1}\right)\right|} \frac{(1+|\xi|)^{2 \alpha} d \xi}{\left(1+\left|\lambda_{1}\right|^{\frac{2}{3}}\right)\left(1+\left|\lambda_{2}\right|^{\frac{2}{3}}\right)} \\
& \quad \leq \frac{C}{\left(1+\left|\lambda_{1}\right|\right)^{\frac{1}{3}-\frac{2 \alpha}{3}}\left(1+\left|\lambda_{2}\right|\right)^{\frac{2}{3}}} \leq C\left(1+\left|\lambda_{2}\right|\right)^{\frac{2 \alpha}{3}-1} .
\end{aligned}
$$

In $I I_{2}$, note that $\xi-\mid \mu\left(\lambda_{1} \mid \geq 0\right.$, so that

$$
\begin{aligned}
I I_{2}\left(\lambda_{1}, \lambda_{2}\right) & \leq \int_{\delta\left|\lambda_{2}\right|}^{2\left|\mu\left(\lambda_{2}\right)\right|} \frac{(1+|\xi|)^{2 \alpha-2} d \xi}{\left(1+\left|\lambda_{2}\right|^{\frac{2}{3}}\right)+\left(2 \xi-\left|\mu\left(\lambda_{2}\right)\right|\right)^{2}} \\
& \leq C\left(1+\left|\lambda_{2}\right|\right)^{\frac{2 \alpha}{3}-1} .
\end{aligned}
$$

For $I I_{3}$, it follows directly that

$$
I I_{3}\left(\lambda_{1}, \lambda_{2}\right) \leq \int_{2\left|\mu\left(\lambda_{2}\right)\right|}^{\infty} \frac{1}{(1+|\xi|)^{4-2 \alpha}} d \xi \leq C\left(1+\left|\lambda_{2}\right|\right)^{\frac{2 \alpha}{3}-1}
$$

Thus, for $0<\lambda_{1} \leq \lambda_{2}$ and $\lambda_{1}$ large,

$$
\left|I I\left(\lambda_{1}, \lambda_{2}\right)\right| \leq C\left(1+\left|\lambda_{2}\right|\right)^{\frac{2 \alpha}{3}-1} .
$$


These three inequalities imply

$$
\begin{aligned}
& \int_{0}^{\infty}(1+|\xi|)^{2 \alpha}\left|\mathcal{P}_{21-1}(\xi, t)\right|^{2} d \xi \leq C \int_{0}^{\infty} \int_{0}^{\lambda_{2}} \phi_{2}\left(\mu\left(\lambda_{1}\right)\right) \phi_{2}\left(\mu\left(\lambda_{2}\right)\right)\left|\hat{h}\left(\lambda_{1}\right) \hat{h}\left(\lambda_{2}\right)\right| \\
& \times\left|\lambda_{1}\right|^{\frac{1}{3}}\left|\lambda_{2}\right|^{\frac{1}{3}}\left(1+\left|\lambda_{2}\right|\right)^{\frac{2 \alpha}{3}-1} d \lambda_{1} d \lambda_{2} \\
& =C \int_{0}^{\infty} \int_{0}^{\lambda_{2}} \frac{\phi_{2}\left(\mu\left(\lambda_{1}\right)\right) \phi_{2}\left(\mu\left(\lambda_{2}\right)\right)\left(1+\left|\lambda_{1}\right|\right)^{\frac{1+\alpha}{3}}\left(1+\left|\lambda_{2}\right|\right)^{\frac{1+\alpha}{3}}}{\left(1+\left|\lambda_{1}\right|\right)^{\frac{\alpha}{3}}\left(1+\left|\lambda_{2}\right|\right)^{1-\frac{\alpha}{3}}}\left|\hat{h}\left(\lambda_{1}\right) \hat{h}\left(\lambda_{2}\right)\right| d \lambda_{1} d \lambda_{2} \\
& \leq C \int_{0}^{\infty} \int_{0}^{\lambda_{2}}\left|\lambda_{1}\right|^{-\frac{\alpha}{3}}\left|\lambda_{2}\right|^{-1+\frac{\alpha}{3}}\left(1+\left|\lambda_{1}\right|\right)^{\frac{1+\alpha}{3}}\left(1+\left|\lambda_{2}\right|\right)^{\frac{1+\alpha}{3}}\left|\hat{h}\left(\lambda_{1}\right) \hat{h}\left(\lambda_{2}\right)\right| d \lambda_{1} d \lambda_{2} \\
& \leq C\left\|\left(1+\left|\lambda_{1}\right|\right)^{\frac{1+\alpha}{3}}\left|\hat{h}\left(\lambda_{1}\right)\right|\right\|_{L^{2}}\left\|\left(1+\left|\lambda_{2}\right|\right)^{\frac{1+\alpha}{3}}\left|\hat{h}\left(\lambda_{2}\right)\right|\right\|_{L^{2}}
\end{aligned}
$$

$(3.2 \underline{20}) C\|h\|_{H^{\frac{1+\alpha}{3}}}^{2}$,

if $\frac{\alpha}{3}<\frac{1}{2}$, or $\alpha<\frac{3}{2}$ (see e.g. page 245 in [24]).

Similarly, for $\mathcal{P}_{21-2}$, we need only study

$$
I I I\left(\lambda_{1}, \lambda_{2}\right)=\int_{0}^{\infty}(1+|\xi|)^{2 \alpha} D_{21}\left(\xi, \lambda_{1}\right) D_{21}\left(\xi, \lambda_{2}\right) d \xi
$$

where

$$
D_{21}(\xi, \lambda)=2 \int_{0}^{\infty} \frac{\eta^{2} \Theta(\eta, \lambda)}{\xi\left(\xi^{2}-\eta^{2}\right)} q_{2}(\eta, \lambda) d \eta+(1+\nu(\xi) \omega(\lambda)) \int_{0}^{\infty} \frac{\eta^{2}(1-\Theta(\eta, \lambda))}{\xi\left(\xi^{2}-\eta^{2}\right)} q_{2}(\eta, \lambda) d \eta .
$$

Note that $D_{21}=D_{2} \sqrt{\mu^{2}(\lambda)-4}$ with $D_{2}$ as defined in (3.8). Following the analysis of $I I\left(\lambda_{1}, \lambda_{2}\right)$, fix $\delta_{1}>0$ small and rewrite $I I I$ in the form

$$
\begin{aligned}
& I I I\left(\lambda_{1}, \lambda_{2}\right)=\left(\int_{0}^{\delta_{1}\left|\mu\left(\lambda_{1}\right)\right|}+\int_{\delta_{1}\left|\mu\left(\lambda_{1}\right)\right|}^{\min \left\{\delta_{1}\left|\mu\left(\lambda_{2}\right)\right|, 100\left|\mu\left(\lambda_{1}\right)\right|\right\}}+\int_{\min \left\{\delta_{1}\left|\mu\left(\lambda_{2}\right)\right|, 100\left|\mu\left(\lambda_{1}\right)\right|\right\}}^{\delta_{1}\left|\mu\left(\lambda_{2}\right)\right|}+\int_{\max \left\{\delta\left|\mu\left(\lambda_{2}\right)\right|, 100\left|\mu\left(\lambda_{1}\right)\right|\right\}}^{\infty}+\int_{100\left|\mu\left(\lambda_{2}\right)\right|}^{\infty}(\cdots) d \xi\right. \\
& +\int_{\delta_{1}\left|\mu\left(\lambda_{2}\right)\right|}^{\max \left\{\delta_{1}\left|\mu\left(\lambda_{2}\right)\right|, 100\left|\mu\left(\lambda_{1}\right)\right|\right\}}+\int^{100\left|\mu\left(\lambda_{2}\right)\right|} \\
& \quad I I I_{1}\left(\lambda_{1}, \lambda_{2}\right)+I I I_{2}\left(\lambda_{1}, \lambda_{2}\right)+I I I_{3}\left(\lambda_{1}, \lambda_{2}\right)+I I I_{4}\left(\lambda_{1}, \lambda_{2}\right) \\
& \quad+I I I_{5}\left(\lambda_{1}, \lambda_{2}\right)+I I I_{6}\left(\lambda_{1}, \lambda_{2}\right) .
\end{aligned}
$$

From the estimates of $D_{2}$ in (3.9), (3.10) and (3.11), it follows that for $\xi \geq 100 \mu(\lambda)$, say,

$$
D_{21}(\xi, \lambda) \leq \frac{C \mu^{2}(\lambda)(1+|\ln | \xi / \mu(\lambda) \mid)}{\xi^{3}}
$$

whereas, for $\delta_{1} \mu(\lambda) \leq \xi \leq 100 \mu(\lambda)$

$$
D_{21}(\xi, \lambda) \leq \frac{C}{\mu(\lambda)}
$$


and for $0 \leq \xi \leq \delta_{1} \mu(\lambda)$

$$
D_{21}(\xi, \lambda) \leq \frac{C|\xi|(1+|\ln | \xi / \mu(\lambda)||)}{\mu^{2}(\lambda)} .
$$

The estimate for $I I I_{1}$ is obtained as follows:

$$
\begin{aligned}
& I I I_{1}\left(\lambda_{1}, \lambda_{2}\right) \\
& \leq C \int_{0}^{\delta_{1} \mu\left(\lambda_{1}\right)} \frac{(1+|\xi|)^{2 \alpha} \xi^{2}\left(1+|\ln | \xi / \mu\left(\lambda_{1}\right)||\right)\left(1+|\ln | \xi / \mu\left(\lambda_{2}\right) \mid\right)}{\mu^{2}\left(\lambda_{1}\right) \mu^{2}\left(\lambda_{2}\right)} d \xi \\
& =C \int_{0}^{\delta_{1}} \frac{\left(1+\left|\mu\left(\lambda_{1}\right) w\right|\right)^{2 \alpha} \mu\left(\lambda_{1}\right) w^{2}(1+|\ln | w||)\left(1+|\ln | \mu\left(\lambda_{1}\right) w / \mu\left(\lambda_{2}\right)||\right) \mid}{\mu^{2}\left(\lambda_{2}\right)} d \xi \\
& \leq C \mu^{1+2 \alpha}\left(\lambda_{1}\right) \mu^{-2}\left(\lambda_{2}\right) \leq C\left(1+\left|\lambda_{1}\right|\right)^{\frac{1+2 \alpha}{3}}\left(1+\left|\lambda_{2}\right|\right)^{-\frac{2}{3}} .
\end{aligned}
$$

Continuing as in the estimate of (3.20) yields the same inequality, of course with a different constant, as appeared in (3.20) provided $-\frac{\alpha}{3}<\frac{1}{2}$, which is to say $\alpha>-\frac{3}{2}$.

For $I I I_{2}$, we have

$$
\begin{aligned}
& \operatorname{III}_{2}\left(\lambda_{1}, \lambda_{2}\right) \\
& \leq C \int_{\delta_{1} \mu\left(\lambda_{1}\right)}^{\min \left\{\delta_{1} \mu\left(\lambda_{2}\right), 100 \mu\left(\lambda_{1}\right)\right\}} \frac{(1+|\xi|)^{2 \alpha}|\xi|\left(1+|\ln | \xi / \mu\left(\lambda_{2}\right)||\right)}{\mu\left(\lambda_{1}\right) \mu^{2}\left(\lambda_{2}\right)} d \xi \\
& \leq C\left(\mu\left(\lambda_{1}\right)\right)^{-1}\left(\mu\left(\lambda_{2}\right)\right)^{-2} \int_{\delta_{1} \mu\left(\lambda_{1}\right)}^{100 \mu\left(\lambda_{1}\right)}(1+|\xi|)^{1+2 \alpha}\left(1+|\ln | \xi / \mu\left(\lambda_{2}\right) \mid\right) d \xi \\
& \leq C\left(1+\left|\lambda_{1}\right|\right)^{\frac{1+2 \alpha}{3}}\left(1+\left|\lambda_{2}\right|\right)^{-\frac{2}{3}}
\end{aligned}
$$

which again gives the estimate (3.20) if $\alpha>-\frac{3}{2}$.

For $I I I_{3}$, note that it is only required to consider the case wherein

$$
100 \mu\left(\lambda_{1}\right)=\min \left\{\delta_{1} \mu\left(\lambda_{2}\right), 100 \mu\left(\lambda_{1}\right)\right\} .
$$

In this situation,

$$
\begin{aligned}
& \operatorname{III}_{3}\left(\lambda_{1}, \lambda_{2}\right) \\
& \leq C \int_{100 \mu\left(\lambda_{1}\right)}^{\delta_{1} \mu\left(\lambda_{2}\right)} \frac{(1+|\xi|)^{2 \alpha} \mu^{2}\left(\lambda_{1}\right)\left(1+|\ln | \xi / \mu\left(\lambda_{1}\right)||\right)\left(1+|\ln | \xi / \mu\left(\lambda_{2}\right)||\right)}{\xi^{2} \mu^{2}\left(\lambda_{2}\right)} d \xi \\
& \leq C\left(\mu\left(\lambda_{1}\right)\right)^{2}\left(\mu\left(\lambda_{2}\right)\right)^{-2} \int_{100 \mu\left(\lambda_{1}\right)}^{\infty}(1+|\xi|)^{2 \alpha-2}\left(1+|\ln | \xi / \mu\left(\lambda_{1}\right)||\right) \\
& \quad \times\left(1+|\ln | \xi / \mu\left(\lambda_{2}\right) \mid\right) d \xi \\
& \leq C\left(\mu\left(\lambda_{1}\right)\right)^{2 \alpha+1}\left(\mu\left(\lambda_{2}\right)\right)^{-2} \leq C\left(1+\left|\lambda_{1}\right|\right)^{\frac{1+2 \alpha}{3}}\left(1+\left|\lambda_{2}\right|\right)^{-\frac{2}{3}},
\end{aligned}
$$

if $2 \alpha-1<0$, which is to say, $\alpha<\frac{1}{2}$. This gives the estimate (3.20) as before. 
Again, in $\mathrm{II}_{4}$, it is only necessary to consider the case wherein

$$
\max \left\{\delta_{1} \mu\left(\lambda_{2}\right), 100 \mu\left(\lambda_{1}\right)\right\}=100 \mu\left(\lambda_{1}\right) .
$$

In this case, $\delta_{1} \mu\left(\lambda_{2}\right) \leq 100 \mu\left(\lambda_{1}\right)$, which implies that $0<C_{0} \lambda_{2} \leq \lambda_{1} \leq \lambda_{2}$ for some $C_{0}>0$. Thus, it follows that

$$
\begin{aligned}
I I I_{4}\left(\lambda_{1}, \lambda_{2}\right) & \leq C \int_{\delta_{1} \mu\left(\lambda_{2}\right)}^{100 \mu\left(\lambda_{1}\right)} \frac{(1+|\xi|)^{2 \alpha} \mu^{-1}\left(\lambda_{1}\right) \mu^{-1}\left(\lambda_{2}\right)}{d} \xi \\
& \leq C\left(\mu\left(\lambda_{1}\right)\right)^{-1}\left(\mu\left(\lambda_{2}\right)\right)^{-1} \int_{\delta_{1} \mu\left(\lambda_{1}\right)}^{100 \mu\left(\lambda_{1}\right)}(1+|\xi|)^{2 \alpha} d \xi \\
& \leq C\left(\mu\left(\lambda_{1}\right)\right)^{2 \alpha}\left(\mu\left(\lambda_{2}\right)\right)^{-1} \leq C\left(1+\left|\lambda_{1}\right|\right)^{(2 \alpha) / 3}\left(1+\left|\lambda_{2}\right|\right)^{-1 / 3} \\
& \leq C\left(1+\left|\lambda_{1}\right|\right)^{\frac{1+2 \alpha}{3}}\left(1+\left|\lambda_{2}\right|\right)^{-\frac{2}{3}} .
\end{aligned}
$$

For $I I I_{5}$, argue as follows:

$$
\begin{aligned}
& I_{5}\left(\lambda_{1}, \lambda_{2}\right) \\
& \leq C \int_{\max \left\{\delta_{1} \mu\left(\lambda_{2}\right), 100 \mu\left(\lambda_{1}\right)\right\}}^{100 \mu\left(\lambda_{2}\right)} \frac{(1+|\xi|)^{2 \alpha} \mu^{2}\left(\lambda_{1}\right)\left(1+|\ln | \xi / \mu\left(\lambda_{1}\right) \mid\right)}{\xi^{3} \mu\left(\lambda_{2}\right)} d \xi \\
& \leq C\left(\mu\left(\lambda_{1}\right)\right)^{2}\left(\mu\left(\lambda_{2}\right)\right)^{-1} \int_{\delta_{1} \mu\left(\lambda_{2}\right)}^{100 \mu\left(\lambda_{2}\right)}(1+|\xi|)^{2 \alpha-3}\left(1+|\ln | \xi / \mu\left(\lambda_{1}\right) \mid\right) d \xi \\
& \leq C\left(\mu\left(\lambda_{1}\right)\right)^{2}\left(\mu\left(\lambda_{2}\right)\right)^{2 \alpha-3}\left(\mu\left(\lambda_{2}\right) / \mu\left(\lambda_{1}\right)\right)^{1-2 \alpha} \\
& \leq C\left(1+\left|\lambda_{1}\right|\right)^{\frac{1+2 \alpha}{3}}\left(1+\left|\lambda_{2}\right|\right)^{-\frac{2}{3}}
\end{aligned}
$$

if $\alpha<\frac{1}{2}$. Similarly,

$$
\begin{aligned}
& \operatorname{III}_{6}\left(\lambda_{1}, \lambda_{2}\right) \\
\leq & C \int_{100 \mu\left(\lambda_{2}\right)}^{\infty} \frac{(1+|\xi|)^{2 \alpha} \mu^{2}\left(\lambda_{1}\right) \mu^{2}\left(\lambda_{2}\right)\left(1+|\ln | \xi / \mu\left(\lambda_{1}\right) \mid\right)\left(1+|\ln | \xi / \mu\left(\lambda_{2}\right) \mid\right)}{\xi^{6}} d \xi \\
\leq & C\left(\mu\left(\lambda_{1}\right) \mu\left(\lambda_{2}\right)\right)^{2} \int_{100 \mu\left(\lambda_{2}\right)}^{\infty}(1+|\xi|)^{2 \alpha-6}\left(1+|\ln | \xi / \mu\left(\lambda_{1}\right) \mid\right)\left(1+|\ln | \xi / \mu\left(\lambda_{2}\right) \mid\right) d \xi \\
\leq & C\left(\mu\left(\lambda_{1}\right)\right)^{2}\left(\mu\left(\lambda_{2}\right)\right)^{2 \alpha-3}\left(\mu\left(\lambda_{2}\right) / \mu\left(\lambda_{1}\right)\right)^{1-2 \alpha} \\
\leq & C\left(1+\left|\lambda_{1}\right|\right)^{\frac{1+2 \alpha}{3}}\left(1+\left|\lambda_{2}\right|\right)^{-\frac{2}{3}},
\end{aligned}
$$

if $\alpha<\frac{1}{2}$. The proof is complete. 


\section{Well-posedness}

In this section, the well-posedness of the IBVP

$$
\left.\begin{array}{l}
u_{t}+u_{x}+u u_{x}+u_{x x x}=0, \quad \text { for } x, t \geq 0, \\
u(x, 0)=\phi(x), \quad u(0, t)=h(t) .
\end{array}\right\}
$$

is studied in the space $H^{s}\left(R^{+}\right) \times H_{l o c}^{\frac{s+1}{3}}\left(R^{+}\right)$. To state precisely our results, we introduce the following notation and Bourgain-type spaces. For any given $s \in R$, $0 \leq b \leq 1,0 \leq \alpha \leq 1$ and function $w \equiv w(x, t): R^{2} \rightarrow R, \Lambda_{s, b}(w)$ and $\lambda_{\alpha}(w)$ are defined as in Section 3. In addition, define

$$
\begin{aligned}
& \mathcal{G}_{s}(w)=\left(\int_{-\infty}^{\infty}(1+|\xi|)^{2 s}\left(\int_{-\infty}^{\infty} \frac{|\hat{w}(\xi, \tau)|}{1+\left|\tau-\left(\xi^{3}-\xi\right)\right|} d \tau\right)^{2} d \xi\right)^{1 / 2} \\
& \mathcal{Q}_{s, b}(w)=\left(\int_{-\infty}^{\infty} \int_{-\infty}^{\infty}(1+|\xi|)^{2 s} \frac{|\hat{w}(\xi, \tau)|^{2}}{\left(1+\left|\tau-\left(\xi^{3}-\xi\right)\right|\right)^{2 b}} d \tau d \xi\right)^{1 / 2}
\end{aligned}
$$

and

$$
\mathcal{P}_{\alpha}(w)=\left(\int_{-\infty}^{\infty} \int_{|\xi| \leq 1} \frac{|\hat{w}(\xi, \tau)|^{2}}{(1+|\tau|)^{2(1-\alpha)}} d \tau d \xi\right)^{1 / 2} .
$$

Let $X_{s, b}$ be the space of all functions $w$ satisfying

$$
\|w\|_{X_{s, b}}:=\Lambda_{s, b}(w)<\infty
$$

(see the definition in (3.2)) while $Y_{s, b}$ is the space of all $w$ satisfying

$$
\|w\|_{Y_{s, b}}:=\left(\mathcal{G}_{s}^{2}(w)+\mathcal{Q}_{s, b}^{2}(w)\right)^{1 / 2}<\infty .
$$

In addition, let $X_{s, b}^{\alpha}$ be the space of all functions $w$ satisfying

$$
\|w\|_{X_{s, b}^{\alpha}}:=\left(\Lambda_{s, b}^{2}(w)+\lambda_{\alpha}^{2}(w)\right)^{1 / 2}<\infty
$$

(again, see the definition in (3.2)) and let $Y_{s, b}^{\alpha}$ be the space of all $w$ satisfying

$$
\|w\|_{Y_{s, b}^{\alpha}}:=\left(\mathcal{P}_{\alpha}^{2}(w)+\mathcal{G}_{s}^{2}(w)+\mathcal{Q}_{s, b}^{2}(w)\right)^{1 / 2}<\infty .
$$

The spaces $X_{s, b}, Y_{s, b}, X_{s, b}^{\alpha}$ and $Y_{s, b}^{\alpha}$ are all Banach spaces. Note that $X_{s, b}$ and $X_{s, b}^{\alpha}$ are equivalent when $b \geq \alpha$. The spaces $Y_{s, b}$ and $X_{s,-b}$ are also equivalent when $b<\frac{1}{2}$. Define also

with the norm

$$
\mathcal{X}_{s, b}^{\alpha} \equiv C\left(R ; H^{s}(R)\right) \cap X_{s, b}^{\alpha}
$$

$$
\|w\|_{\mathcal{X}_{s, b}^{\alpha}}=\left(\sup _{t \in R}\|w(\cdot, t)\|_{H^{s}(R)}^{2}+\|w\|_{X_{s, b}^{\alpha}}^{2}\right)^{1 / 2} .
$$

By their definitions,

$$
X_{s, b_{1}}^{\alpha_{1}} \subset X_{s, b_{2}}^{\alpha_{2}} \quad \text { if } \alpha_{1} \leq \alpha_{2} \text { and } b_{1} \leq b_{2},
$$

whereas

$$
Y_{s, b_{2}}^{\alpha_{2}} \subset Y_{s, b_{1}}^{\alpha_{1}} \quad \text { if } \alpha_{1} \geq \alpha_{2} \text { and } b_{1} \leq b_{2} .
$$


The above Bourgain-type spaces are defined for functions posed on the whole plane $R \times R$. However, the IBVP (4.1) is posed on the quarter plane $R^{+} \times R^{+}$and we are seeking its solution in the space $C\left(R^{+}: H^{s}\left(R^{+}\right)\right)$corresponding to a given initial value in the space $H^{s}\left(R^{+}\right)$and boundary data in the space $H_{l o c}^{\frac{s+1}{3}}\left(R^{+}\right)$. It is thus natural to consider restricted versions of the above Bourgain-type spaces to the quarter plane $R^{+} \times R^{+}$. Let $\Omega$ denote a subinterval of $R$; define a restricted version of the Bourgain space $X_{s, b}$ to the domain $R^{+} \times \Omega$ as follows:

$$
X_{s, b}\left(R^{+} \times \Omega\right)=\left.X_{s, b}\right|_{R^{+} \times \Omega}
$$

with the quotient norm

$$
\|u\|_{X_{s, b}\left(R^{+} \times \Omega\right)} \equiv \inf _{w \in X_{s, b}}\left\{\|w\|_{X_{s, b}}: w(x, t)=u(x, t) \text { on } R^{+} \times \Omega\right\}
$$

for any given function $u(x, t)$ defined on $R^{+} \times \Omega$. Of course, it is clear that $\|u\|_{X_{s, b}}=$ $\|u\|_{X_{s, b}(R \times R)}$. The spaces $Y_{s, b}\left(R^{+} \times \Omega\right), X_{s, b}^{\alpha}\left(R^{+} \times \Omega\right), Y_{s, b}^{\alpha}\left(R^{+} \times \Omega\right)$ and $\mathcal{X}_{s, b}^{\alpha}\left(R^{+} \times \Omega\right)$ are defined similarly. Naturally, if $\tilde{w}$ is any extension of $w$ in $X_{s, b}\left(R^{+} \times \Omega\right)$ to $X_{s, b}(R \times R)$, then $\|\tilde{w}\|_{X_{s, b}(R \times R)} \geq\|w\|_{X_{s, b}\left(R^{+} \times \Omega\right)}$.

For the IBVP (4.1), we have the following well-posedness result.

TheOREM 4.1. Let $s \in\left(-\frac{3}{4}, 1\right), T>0$ and $r>0$ be given. There exist $T^{*}$ with $0<T^{*} \leq T$ and $b$ with $0<b<\frac{1}{2}$ such that

(i) if $\phi \in H^{s}\left(R^{+}\right)$and $h \in H_{l o c}^{\frac{s+1}{3}}\left(R^{+}\right)$satisfy

$$
\|\phi\|_{H^{s}\left(R^{+}\right)}+\|h\|_{H^{\frac{s+1}{3}}(0, T)} \leq r
$$

and

$$
\phi(0)=h(0)
$$

when $s>\frac{1}{2}$, then the $\operatorname{IBVP}$ (4.1) admits a unique solution $u \in \mathcal{X}_{s, \frac{1}{2}}^{\alpha}\left(R^{+} \times\right.$ $\left.\left(0, T^{*}\right)\right)$ for some $\alpha>\frac{1}{2}$;

(ii) if $\phi \in H^{s}\left(R^{+}\right)$and $h \in H_{l o c}^{\frac{3 b-1 / 2+s}{3}}(R)$ satisfy

$$
\|\phi\|_{H^{s}\left(R^{+}\right)}+\|h\|_{H^{\frac{3 b-1 / 2+s}{3}}(0, T)} \leq r
$$

and

$$
\phi(0)=h(0)
$$

when $s>\frac{1}{2}$, then the IBVP (4.1) admits a unique solution $u \in \mathcal{X}_{s, b}^{\alpha}\left(R^{+} \times\right.$ $\left.\left(0, T^{*}\right)\right)$ for some $\alpha>\frac{1}{2}$.

Moreover, the solution $u$ depends continuously on $\phi$ and $h$ in the corresponding spaces.

Remarks: The result presented in part (i) of the above theorem is slightly stronger than that of Colliander and Kenig [12] and Holmer [25] in the sense that $u$ is the restriction to $R^{+} \times\left(0, T^{*}\right)$ of a function $w \in \mathcal{X}_{s, \frac{1}{2}}^{\alpha}$ instead of $w \in \mathcal{X}_{s, b}^{\alpha}$ for some $b$ strictly less than $\frac{1}{2}$. The result presented in part (ii) shows a boundary smoothing since the boundary value $h$ is only required to lie in the space $H_{l o c}^{\frac{3 b-1 / 2+s}{3}}\left(R^{+}\right)$and

$$
\frac{3 b-1 / 2+s}{3}<\frac{s+1}{3}
$$

when $b<\frac{1}{2}$. 
The proof of Theorem 4.1 is based on the results expounded in Section 3 and the following lemmas whose proofs can be found in $[\mathbf{3 3}, \mathbf{3 4}, \mathbf{1 1}, \mathbf{1 2}, \mathbf{4}]$ or derived from them in a straightforward way. For the convenience of the reader, we provide in the Appendix brief indications of the proofs of the results used here. In the lemmas given below, $\psi(t), \sigma(t) \in C_{0}^{\infty}(R)$ with $\psi=1$ on $[-1,1]$ and supp $\psi \subset(-2,2), \sigma=1$ on supp $\psi$ and supp $\sigma \subset(-2,2)$ also.

Lemma 4.2. Assume that $0<\delta<1,-\infty<s<\infty, 0<b \leq 1$ and $\alpha \in\left(\frac{1}{2}, 1\right]$. Then there exists a constant $C$ depending only on $s, b, \alpha$ and $\psi$ such that

$$
\begin{gathered}
\left\|\psi\left(\delta^{-1} t\right) W_{R}(t) \phi\right\|_{X_{s, b}^{\alpha}} \leq C \delta^{\frac{1-2 b^{*}}{2}}\|\phi\|_{H^{s}(R)}, \\
\left\|\psi\left(\delta^{-1} t\right) h\right\|_{X_{s, b}^{\alpha}} \leq C \delta^{\frac{1-2 b^{*}}{2}}\|h\|_{X_{s, b}^{\alpha}},
\end{gathered}
$$

and

$$
\left\|\psi\left(\delta^{-1} t\right) \int_{0}^{t} W_{R}\left(t-t^{\prime}\right) f\left(t^{\prime}\right) d t^{\prime}\right\|_{X_{s, b}^{\alpha}} \leq C \delta^{\frac{1-2 b^{*}}{2}}\|f\|_{Y_{s, 1-b}^{1-\alpha}} .
$$

Here $b^{*}$ is any number larger than $b$.

LEMma 4.3. (a) For $-\infty<s<\infty$, there exists a constant $C$ depending only on s such that

$$
\sup _{x \in R}\left\|W_{R}(t) \phi\right\|_{H_{t}^{\frac{s+1}{3}}(R)} \leq C\|\phi\|_{H^{s}(R)}
$$

for any $\phi \in H^{s}(R)$.

(b) For $-1 \leq s<\infty$, there exists a constant $C$ depending only on $\psi$ and $s$ such that

$$
\sup _{x \in R}\left\|\psi(t) W_{R}(t) \phi\right\|_{H_{t}^{\frac{s+1}{3}}(R)} \leq C\|\phi\|_{H^{s}(R)}
$$

for any $\phi \in H^{s}(R)$.

LEMMA 4.4. Let $0 \leq b<\frac{1}{2},-1 \leq s<\infty$ and define

$$
w(x, t)=\int_{0}^{t}\left[W_{R}\left(t-t^{\prime}\right) f\left(\cdot, t^{\prime}\right)\right](x) d t^{\prime} .
$$

For $\psi$ as described above, there exists $C$ depending only on $b, s$ and $\psi$ such that

$$
\sup _{x \in R}\|\psi(t) w(x, t)\|_{H_{t}^{\frac{s+1}{3}}(R)} \leq C\|f\|_{Y_{s, b}} .
$$

In addition, for $-\infty<s<\infty, 0 \leq b<\frac{1}{2}$,

$$
\sup _{t \in R}\|\psi(t) w(x, t)\|_{H_{x}^{s}(R)} \leq C\|f\|_{Y_{s, b}}
$$

LEMMA 4.5. Given $s>-\frac{3}{4}$, there exist $b=b(s)<\frac{1}{2}, \alpha=\alpha(s)>\frac{1}{2}$ and $C, \mu>0$ such that

$$
\left\|\partial_{x}(u v)\right\|_{Y_{s, b}^{\alpha}} \leq C T^{\mu}\|u\|_{X_{s, b}^{\alpha}}\|v\|_{X_{s, b}^{\alpha}}
$$

for any $u, v \in X_{s, b}^{\alpha}$ with compact support in $[-T, T]$. 
The bilinear estimate (4.9) with $\mu=0$ was established by Colliander and Kenig [12], and Holmer [25] for any $u, v \in X_{s, b}^{\alpha}$. In addition, one may observe by studying Holmer's analysis that for $s \in\left(-\frac{3}{4},-\frac{1}{2}\right)$, it follows that $\max \left\{\frac{2}{3}+\frac{s}{3}, \frac{5}{12}-\frac{s}{9}\right\} \leq b<$ $\frac{1}{2}$ in the above lemma. As one can see, $b \rightarrow \frac{1}{2}$ as $s \rightarrow-\frac{3}{4}$ or $-\frac{1}{2}$. The bilinear estimate presented in the following lemma gives an indication of how small a value of $b$ one can choose for $s$ in the range of $\left(-\frac{9}{16}, 0\right)$.

Lemma 4.6. Let

$$
w(x, t)=\sigma^{2}\left(\delta^{-1} t\right) \partial_{x}(u(x, t) v(x, t))
$$

where $u, v \in X_{s, b}^{\alpha}$. For any given $s$ with $0<s<\frac{9}{16}$, choose $b_{1}, b$ and $\alpha$ by

$$
\max \left\{\frac{1-s}{2}, \frac{7}{16}\right\}<b_{1}<\frac{1}{2}, \quad \frac{7}{16}<b<\frac{1}{2}
$$

and suppose

$$
\frac{1}{2}<\alpha<\frac{1+6 b}{16 b} .
$$

Then there exists a constant $C$ such that

$$
\|w\|_{Y_{-s, b_{1}}^{\alpha}} \leq C \delta^{\theta_{0}}\|u\|_{X_{-s, b}^{\alpha}}\|v\|_{X_{-s, b}^{\alpha}}
$$

for any $u, v \in X_{-s, b}^{\alpha}$ and $-1 \leq \delta \leq 1$, where

$$
\theta_{0}=1-2 \alpha+\frac{1-2 b}{8 b}>0 .
$$

Next, we consider the non-homogeneous linear problem

$$
\left.\begin{array}{l}
u_{t}+u_{x}+u_{x x x}=0, \quad \text { for } x, t \geq 0, \\
u(x, 0)=0, \quad u(0, t)=h(t) .
\end{array}\right\}
$$

Recall its solution may be represented in the form

$$
u(x, t)=\left[\mathcal{W}_{b d r}(t) h\right](x)
$$

for $x, t \geq 0$ as explained in Section 2 .

Lemma 4.7. For a given pair $(b, s)$ satisfying

$$
\left\{\begin{array}{l}
0 \leq b<\frac{1}{2}-\frac{s}{3} \quad \text { if } s \leq 0, \text { or } \\
0 \leq b<\frac{5}{6}-\frac{s}{3} \quad \text { if }-\frac{1}{2}<s<1,
\end{array}\right.
$$

and a given $\alpha \in\left(\frac{1}{2}, 1\right)$, there exists a constant $C$ such that for any $T>0$ and any $h \in H_{0}^{\frac{s+1}{3}}(0, T)$, the corresponding solution u of (4.10) belongs to the restricted Bourgain space $\mathcal{X}_{s, b}^{\alpha}\left(R^{+} \times(0, T)\right)$ and satisfies

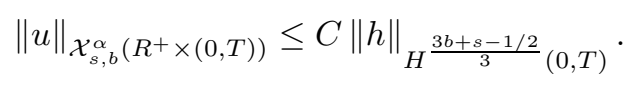


Proof: For $T>0$, let $h_{1} \in H^{\frac{s+1}{3}}\left(R^{+}\right)$be such that $h_{1} \equiv h$ in the space $H^{\frac{s+1}{3}}(0, T)$ and let $\psi_{1} \in C_{0}^{\infty}(R)$ be such that $\psi_{1}(t)=1$ for any $t \in[0, T]$. Define

$$
u_{1}(x, t)= \begin{cases}{\left[\mathcal{B} I_{m 1}(t) h_{1}\right](x)} & \text { if } 0 \leq b<\frac{1}{2}-\frac{s}{3} \text { and } s<0, \\ {\left[\mathcal{B} I_{m 2}(t) h_{1}\right](x)} & \text { if } 0 \leq b<\frac{5}{6}-\frac{s}{3} \text { and } 0 \leq s<1,\end{cases}
$$

Observing that

$$
u(x, t)=u_{1}(x, t) \quad \text { for }(x, t) \in R^{+} \times[0, T],
$$

and using Theorem 3.1, Theorem 3.4 and Lemma 3.10, one arrives at the conclusion,

$$
\|u\|_{\mathcal{X}_{s, b}^{\alpha}\left(R^{+} \times(0, T)\right)} \leq\left\|\psi_{1} u_{1}\right\|_{\mathcal{X}_{s, b}^{\alpha}} \leq C\left\|h_{1}\right\|_{H^{\frac{s+1}{3}}\left(R^{+}\right)}
$$

for a constant $C$ depending on $\psi_{1}$ and $s$. Thus, (4.12) follows and the proof is complete.

Consider the same linear equation posed with zero boundary conditions, but non-trivial initial data, viz.

$$
\left.\begin{array}{ll}
u_{t}+u_{x}+u_{x x x}=0, & \text { for } x, t \geq 0, \\
u(x, 0)=\phi(x), & u(0, t)=0 .
\end{array}\right\}
$$

Its solution can be written as

$$
u(x, t)=\left[\mathcal{W}_{c}(t) \phi\right](x)
$$

for $x, t \geq 0$.

Lemma 4.8. Assume that $\frac{1}{2}<\alpha \leq 1$. For a given pair $(b, s)$ satisfying (4.11) and $0 \leq b \leq 1$, there exists a constant $C$ such that for any $T>0$ and any $\phi \in$ $H_{0}^{s}\left(R^{+}\right)$, the corresponding solution $u$ of (4.13) belongs to the restricted Bourgain space $\mathcal{X}_{s, b}^{\alpha}\left(R^{+} \times(0, T)\right)$ and satisfies the bound

$$
\|u\|_{\mathcal{X}_{s, b}^{\alpha}\left(R^{+} \times(0, T)\right)} \leq C\|\phi\|_{H^{s}\left(R^{+}\right)} .
$$

Proof: According to Proposition 2.2, one may write $W_{c}(t) \phi$ as

$$
W_{c}(t) \phi=W_{R}(t) \phi^{*}-\mathcal{W}_{b d r}(t) g
$$

for any $x, t>0$, where $g$ is the trace of $W_{R}(t) \phi^{*}$ at $x=0$ and $\phi^{*} \in H^{s}(R)$ and equals to $\phi$ when restricted on $R^{+}$. The estimate (4.14) follows from Lemma 4.2, Lemma 4.3 and Lemma 4.7.

Now we turn to the forced linear problem

$$
\left.\begin{array}{l}
u_{t}+u_{x}+u_{x x x}=f, \quad \text { for } x, t \geq 0, \\
u(x, 0)=0, \quad u(0, t)=0 .
\end{array}\right\}
$$

Its solution can be written in the form

$$
u(\cdot, t)=\int_{0}^{t} W_{c}(t-\tau) f(\cdot, \tau) d \tau .
$$


LEMmA 4.9. Assume that $-1 \leq s<1, \frac{1}{2}<\alpha \leq 1$ and $0<b<\frac{1}{2}$. For any $T>0$, there is a constant $C$ such that for any $f \in Y_{s, b}^{1-\alpha}\left(R^{+} \times(0, T)\right)$, the corresponding solution $u$ of (4.15) belongs to the space $\mathcal{X}_{s, b}^{\alpha}\left(R^{+} \times(0, T)\right)$ and satisfies the inequality

$$
\|u\|_{\mathcal{X}_{s, b}^{\alpha}\left(R^{+} \times(0, T)\right)} \leq C\|f\|_{Y_{s, b}^{1-\alpha}\left(R^{+} \times(0, T)\right)} .
$$

In addition, there exists a $b^{*} \in\left(0, \frac{1}{2}\right)$ such that if $f \in Y_{s, b^{*}}^{1-\alpha}\left(R^{+} \times(0, T)\right)$, then the corresponding solution $u$ of (4.15) belongs to the space $\mathcal{X}_{s, \frac{1}{2}}^{\alpha}\left(R^{+} \times(0, T)\right)$ and satisfies the bound

$$
\|u\|_{\mathcal{X}_{s, \frac{1}{2}}^{\alpha}\left(R^{+} \times(0, T)\right)} \leq C\|f\|_{Y_{s, b^{*}}^{1-\alpha}\left(R^{+} \times(0, T)\right)} \cdot
$$

Proof: By Proposition 2.3,

$$
u(\cdot, t)=\int_{0}^{t} W_{R}(t-\tau) f(\cdot, \tau) d \tau-\mathcal{W}_{b d r}(t) v
$$

for any $x, t>0$ where $v \equiv v(t)$ is the trace of $\int_{0}^{t} W_{R}(t-\tau) f(\cdot, \tau) d \tau$ at $x=0$. The estimates (4.16) and (4.17) then follow from Lemma 4.2, Lemma 4.4 and Lemma 4.7 by noting that $Y_{s, \frac{1}{2}}^{1-\alpha}\left(R^{+} \times(0, T)\right)$ is a subspace of $Y_{s, b^{*}}^{1-\alpha}\left(R^{+} \times(0, T)\right)$.

The lemma below presents a version of a bilinear estimate in the restricted Bourgain space $X_{s, b}\left(R^{+} \times(0, T)\right)$ which follows directly from Lemma 4.5.

LEMmA 4.10. Given $s>-\frac{3}{4}$, there exist $b=b(s)<\frac{1}{2}, \alpha=\alpha(s)>\frac{1}{2}$ and $C, \mu>0$ such that

$$
\left\|\partial_{x}(u v)\right\|_{Y_{s, b}^{\alpha}\left(R^{+} \times(0, T)\right)} \leq C T^{\mu}\|u\|_{\mathcal{X}_{s, b}^{\alpha}\left(R^{+} \times(0, T)\right.}\|v\|_{\mathcal{X}_{s, b}^{\alpha}\left(R^{+} \times(0, T)\right)}
$$

for any $u, v \in \mathcal{X}_{s, b}^{\alpha}\left(R^{+} \times(0, T)\right)$.

We are now ready to present the proof of Theorem 4.1 .

Proof of Theorem 4.1: By applying Lemmas 4.7-4.10, Theorem 4.1 can be established by the standard contraction mapping principle. The proof is provided only for Part (i). The proof of Part (ii) is entirely similar and so is omitted.

Let $\phi \in H^{s}\left(R^{+}\right)$and $h \in H_{l o c}^{\frac{s+1}{3}}\left(R^{+}\right)$be given with $s \in\left(-\frac{3}{4}, \frac{1}{2}\right]$. For given $\theta$ with $0<\theta \leq 1$ (to be chosen precisely later) and $v, w \in \mathcal{X}_{s, \frac{1}{2}}^{\alpha}\left(R^{+} \times(0, \theta)\right.$ ), define

$$
\mathbf{F}(w)=W_{c}(t) \phi+\mathcal{W}_{b d r}(t) h-\int_{0}^{t} W_{c}(t-\tau)\left(w_{x} w\right)(\tau) d \tau .
$$

Using Lemma 4.7 -Lemma 4.10, it is seen that

$\|\mathbf{F}(w)\|_{\mathcal{X}_{s, \frac{1}{2}}^{\alpha}\left(R^{+} \times(0, \theta)\right)} \leq C_{1}\left(\|\phi\|_{H^{s}\left(R^{+}\right)}+\|h\|_{H^{\frac{s+1}{3}}(0, T)}\right)+C_{2} \theta^{\mu}\|w\|_{\mathcal{X}_{s, \frac{1}{2}}^{\alpha}\left(R^{+} \times(0, \theta)\right)}^{2}$ and

$$
\|\mathbf{F}(v)-\mathbf{F}(w)\|_{\left.\mathcal{X}_{s, \frac{1}{2}}\left(R^{+} \times \theta\right)\right)} \leq C_{2} \theta^{\mu}\|v-w\|_{\mathcal{X}_{s, \frac{1}{2}}\left(R^{+} \times(0, \theta)\right)}\|v+w\|_{\mathcal{X}_{s, \frac{1}{2}}\left(R^{+} \times(0, \theta)\right)}
$$

where the constant $C_{1}$ and $C_{2}$ are independent of $\theta, v$ and $w$. Let $B_{r}$ be the ball in the space $\mathcal{X}_{s, \frac{1}{2}}^{\alpha}\left(R^{+} \times(0, \theta)\right)$ with radius $r$ where

$$
r=2 C_{1}\left(\|\phi\|_{H^{s}\left(R^{+}\right)}+\|h\|_{H^{\frac{s+1}{3}}(0, T)}\right),
$$


and choose $\theta=T^{*}$ small enough that

$$
2 C_{2}\left(T^{*}\right)^{\mu} r \equiv \beta<1 .
$$

Then it follows readily that $\mathbf{F}$ maps $B_{r}$ into itself and that for $w, v \in B_{r}$,

$$
\|\mathbf{F}(w)-\mathbf{F}(v)\|_{\mathcal{X}_{s, \frac{1}{2}}^{\alpha}\left(R^{+} \times\left(0, T^{*}\right)\right)} \leq \beta\|w-v\|_{\mathcal{X}_{s, \frac{1}{2}}^{\alpha}\left(R^{+} \times\left(0, T^{*}\right)\right)} .
$$

Thus, the mapping $\mathbf{F}$ is a contraction on the ball $B_{r}$. The fixed point $u$ of this map $\mathbf{F}$ in $B_{r}$ is the advertised solution. For $s>1 / 2$, we use the integral form $(2.12)$ together with $f=-u u_{x}$ to obtain the result.

It is next demonstrated that there is at most one fixed point of the operator $\mathbf{F}$, whether or not the fixed point is determined by the contraction mapping principle.

LEMma 4.11. Let $-\frac{3}{4}<s<1$ and fix $\phi \in H^{s}\left(R^{+}\right)$and $h \in H_{l o c}^{\frac{s+1}{3}}\left(R^{+}\right)$. Let $\mathbf{F}$ be as defined in (4.19). Suppose $u_{1} \in \mathcal{X}_{s, \frac{1}{2}}^{\alpha}\left(R^{+} \times\left(0, \theta_{1}\right)\right)$ and $u_{2} \in \mathcal{X}_{s, \frac{1}{2}}^{\alpha}\left(R^{+} \times\left(0, \theta_{2}\right)\right)$ both are fixed points of $\mathbf{F}$, where $0<\theta_{1} \leq \theta_{2}<\theta$. Then,

$$
\left.u_{2}\right|_{t \in\left(0, \theta_{1}\right)}=u_{1}
$$

in $\mathcal{X}_{s, \frac{1}{2}}^{\alpha}\left(R^{+} \times\left(0, \theta_{1}\right)\right)$.

Proof: The argument is made by standard energy estimates using the bilinear estimates just derived. Let $w=u_{1}-u_{2}$. Then, it follows that, at least for $0 \leq t \leq \theta_{1}$, $(4.20)$

$$
w=\mathbf{F}\left(u_{1}\right)-\mathbf{F}\left(u_{2}\right)=\frac{1}{2} \int_{0}^{t} W_{c}(t-\tau) \partial_{x}\left(u_{1}^{2}-u_{2}^{2}\right) d \tau=\frac{1}{2} \int_{0}^{t} W_{c}(t-\tau) \partial_{x}\left[\left(u_{1}+u_{2}\right) w\right] d \tau .
$$

The equality

$$
\int_{0}^{t} W_{c}(t-\tau) \partial_{x}\left(u^{2}-v^{2}\right) d \tau=\int_{0}^{t} W_{c}(t-\tau) \partial_{x}[(u+v)(u-v)] d \tau
$$

follows from the fact that it holds for smooth functions $u$ and $v$ and the observation that such functions are dense in $\mathcal{X}_{s, \frac{1}{2}}^{\alpha}\left(R^{+} \times(0, \theta)\right)$. Let $\theta$ be fixed with $0<\theta \leq \theta_{1}$. Using the same analysis that came to the fore in establishing that $\mathbf{F}$ is contractive, it is deduced from (4.20) that

$$
\|w\|_{\mathcal{X}_{s, \frac{1}{2}}^{\alpha}\left(R^{+} \times(0, \theta)\right)} \leq C \theta^{\mu}\left\|u_{1}+u_{2}\right\|_{\mathcal{X}_{s, \frac{1}{2}}^{\alpha}\left(R^{+} \times(0, \theta)\right)}\|w\|_{\mathcal{X}_{s, \frac{1}{2}}^{\alpha}\left(R^{+} \times(0, \theta)\right)}
$$

where $C$ is independent of $u_{1}, u_{2}, \theta$ and $\mu>0$. Continuing this inequality, there obtains

$$
\|w\|_{\mathcal{X}_{s, \frac{1}{2}}^{\alpha}\left(R^{+} \times(0, \theta)\right)} \leq C \theta^{\mu} M\|w\|_{\mathcal{X}_{s, \frac{1}{2}}^{\alpha}\left(R^{+} \times(0, \theta)\right)}
$$

where

$$
M=\max \left\{\left\|u_{1}\right\|_{\mathcal{X}_{s, \frac{1}{2}}^{\alpha}\left(R^{+} \times(0, \theta)\right)},\left\|u_{2}\right\|_{\mathcal{X}_{s, \frac{1}{2}}^{\alpha}\left(R^{+} \times(0, \theta)\right)}\right\} .
$$

Here, use is made of the fact that if $v \in \mathcal{X}_{s, \frac{1}{2}}^{\alpha}\left(R^{+} \times(0, T)\right)$, then if $S<T, v \in$ $\mathcal{X}_{s, \frac{1}{2}}^{\alpha}\left(R^{+} \times(0, S)\right)$ and

$$
\|v\|_{\mathcal{X}_{s, \frac{1}{2}}^{\alpha}\left(R^{+} \times(0, S)\right)} \leq\|v\|_{\mathcal{X}_{s, \frac{1}{2}}^{\alpha}\left(R^{+} \times(0, T)\right)} .
$$


If $\theta$ is chosen small enough, then (4.21) forces $\|w\|_{\mathcal{X}_{s, \frac{1}{2}}^{\alpha}\left(R^{+} \times(0, \theta)\right)}=0$. So, $w=0$ and $u_{1}=u_{2}$ as elements of $\mathcal{X}_{s, \frac{1}{2}}^{\alpha}\left(R^{+} \times(0, \theta)\right)$. Define $\theta_{0}$ to be

$$
\theta_{0}=\sup \left\{\theta>0: u_{1}=u_{2} \in \mathcal{X}_{s, \frac{1}{2}}^{\alpha}\left(R^{+} \times(0, \theta)\right)\right\}
$$

and suppose $\theta_{0}<\theta_{1}$. Then, $u_{1}\left(\cdot, \theta_{0}\right)=u_{2}\left(\cdot, \theta_{0}\right)$ but this is no longer true for some $\theta$ 's with $\theta>\theta_{0}$ arbitrarily close to $\theta_{0}$. Consider again (4.20) and for $\theta>\theta_{0}$, write it in the form

$$
w(\cdot, t)=\frac{1}{2} \int_{0}^{\theta_{0}} W_{c}(t-\tau) \partial_{x}\left[\left(u_{1}+u_{2}\right) w\right] d \tau+\frac{1}{2} \int_{\theta_{0}}^{t} W_{c}(t-\tau) \partial_{x}\left[\left(u_{1}+u_{2}\right) w\right] d \tau .
$$

Because of the choice of $\theta_{0}$, the first term above is zero, and so

$$
w=\frac{1}{2} \int_{\theta_{0}}^{t} W_{c}(t-\tau) \partial_{x}\left[\left(u_{1}+u_{2}\right) w\right] d \tau .
$$

As before,

$$
\|w\|_{\mathcal{X}_{s, \frac{1}{2}}^{\alpha}\left(R^{+} \times\left(\theta_{0}, \theta\right)\right)} \leq C\left(\theta-\theta_{0}\right)^{\mu} M\|w\|_{\mathcal{X}_{s, \frac{1}{2}}^{\alpha}\left(R^{+} \times\left(\theta_{0}, \theta\right)\right)}
$$

and this, too, implies $w=0$ on $\left[\theta_{0}, \theta\right]$ if $\theta$ is chosen sufficiently near $\theta_{0}$. Thus, $\theta_{0}=\theta_{1}$ and

as advertised.

$$
\left.u_{2}\right|_{t \in\left(0, \theta_{1}\right)}=u_{1}
$$

Corollary 4.12. Let $r>s$ lie in the interval $\left(-\frac{3}{4}, 1\right)$. Suppose for some $\theta>0$ $u_{r} \in \mathcal{X}_{r, \frac{1}{2}}^{\alpha}\left(R^{+} \times(0, \theta)\right)$ and $u_{s} \in \mathcal{X}_{s, \frac{1}{2}}^{\alpha}\left(R^{+} \times(0, \theta)\right)$ are both fixed points of $\mathbf{F}$ where $(\phi, h)$ lie in $H^{r}\left(R^{+}\right) \times H_{l o c}^{\frac{r+1}{3}}\left(R^{+}\right)$. Then, $u_{r}=u_{s}$ in $\mathcal{X}_{r, \frac{1}{2}}^{\alpha}\left(R^{+} \times(0, \theta)\right)$.

Proof: Since $\mathcal{X}_{r, b}^{\alpha}\left(R^{+} \times(0, \theta)\right) \subset \mathcal{X}_{s, b}^{\alpha}\left(R^{+} \times(0, \theta)\right)$, it follows that both $u_{r}$ and $u_{s}$ are fixed points of $\mathbf{F}$ in $\mathcal{X}_{s, b}^{\alpha}\left(R^{+} \times(0, \theta)\right)$ and hence must be equal there. As $u_{r}$ lies in the smoother class, so does $u_{s}$

Remark: It is worth noting that if $u$ is a strong solution of the initial-boundaryvalue problem (1.1), which is to say that $u \in C\left([0, T] ; H^{3}\left(R^{+}\right)\right)$for some $T>0$, then $u$ does satisfy the fixed-point problem (4.19), at least for $0 \leq t \leq T$. This follows readily from the formal derivation of (4.19) in Section 2, all of whose steps are rigorously verified in the context of that much regularity. Note, again, that the mapping $\mathbf{F}$ does not depend upon any extension operations, though we have used such operators to represent $\mathbf{F}$ (in a non-unique way) to facilitate its analysis.

The well-posedness result presented in Theorem 4.1 is conditional since the uniqueness is established in the space $\mathcal{X}_{s, b}\left(R^{+} \times(0, T)\right)$ instead of in the space $C\left([0, T] ; H^{s}\left(R^{+}\right)\right)$. Attention is now given to showing the well posedness is unconditional. Toward this end, following the development in [7], it is first proved that the well-posedness result in Theorem 4.1 has the property of persistence of regularity.

Proposition 4.13 (persistence of regularity). For given $s$ with $-\frac{3}{4}<s<1$ and $T>0$, let $\phi \in H^{3}\left(R^{+}\right)$and $h \in H_{\text {loc }}^{\frac{4}{3}}(0, T)$ satisfy the compatibility condition

$$
h(0)=\phi(0) \text {. }
$$


Then the solution $u_{s} \in C\left(\left[0, T_{s}\right] ; H^{s}\left(R^{+}\right)\right), T_{s} \leq T$, corresponding to the auxiliary data $(\phi, h)$ given by Theorem 4.1 has the property that

$$
u_{s} \in C\left(\left[0, T_{s}\right] ; H^{3}\left(R^{+}\right)\right) .
$$

Proof: First, using the global well-posedness theory developed in [4], there is adduced a unique solution $\tilde{u}$ of (1.1) corresponding to the auxiliary data $\phi \in$ $H^{3}\left(R^{+}\right)$and $h \in H_{l o c}^{\frac{4}{3}}\left(R^{+}\right)$which, for every $T>0$, lies in $C\left([0, T] ; H^{3}\left(R^{+}\right)\right) \cap$ $C^{1}\left([0, T] ; L^{2}\left(R^{+}\right)\right)$. To prove that $u_{s}$ lies in $C\left([0, T] ; H^{3}\left(R^{+}\right)\right)$, it suffices to show that $\tilde{u}$, when restricted to $\left[0, T_{s}\right]$, lies in the uniqueness class for $u_{s}$, which is to say, $\tilde{u} \in \mathcal{X}_{s, \frac{1}{2}}^{\alpha}\left(R^{+} \times\left(0, T_{s}\right)\right)$. This follows since, as noted in the remark following Corollary 4.12, $\tilde{u}$ is a fixed point of $\mathbf{F}$. Moreover, by Theorem 4.1, the value $T_{s}$ depends only on $\|(\phi, h)\|_{H^{s}\left(R^{+}\right) \times H^{\frac{s+1}{3}}(0, T)}$. Note also that if $s_{2} \leq s_{1}$, then $T_{s_{1}} \leq T_{s_{2}}$ and $\mathcal{X}_{s_{1}, b}^{\alpha}\left(R^{+} \times(0, T) \subset \mathcal{X}_{s_{2}, b}^{\alpha}\left(R^{+} \times(0, T)\right)\right.$ for any $T>0$. In consequence of these ruminations, it suffices to show $\tilde{u} \in \mathcal{X}_{s, \frac{1}{2}}^{\alpha}\left(R^{+} \times(0, T)\right)$ for some $s$ in the range $-\frac{3}{4}<s<-\frac{1}{2}$. We turn to establishing this fact.

Let $T=2 T_{s}$, say, and let $u_{1}(x, t)$ be the even extension in both $x$ and $t$ of $\tilde{u}$ to the domain $R \times(-T, T)$. Multiply $u_{1}$ by an even cut-off function $\psi_{1}(t)$ which is identically 1 on $\left[-T_{s}, T_{s}\right]$ and whose support lies in $(-T, T)$, and view $U=\psi_{1} u_{1}$ as a function defined on $R \times R$. The Fourier transform of $U$ with respect to $x$ and $t$ is

$$
\hat{U}(\xi, \tau)=4 \int_{0}^{\infty} \int_{0}^{\infty} U(x, t) \cos (x \xi) \cos (t \tau) d x d t
$$

Since $U(x, t) \in C^{1}\left(R^{+} ; L^{2}\left(R^{+}\right)\right)$has a compact support in $t$, an integration by parts gives

which yields

$$
\tau \hat{U}(\xi, \tau)=-4 \int_{0}^{\infty} \int_{0}^{\infty} U_{t}(x, t) \cos (x \xi) \sin (t \tau) d x d t
$$

$$
\begin{aligned}
& \|\tau \hat{U}(\xi, \tau)\|_{L^{2}(R \times R)} \leq C\left\|U_{t}(x, t)\right\|_{L^{2}\left(R^{+} \times R^{+}\right)} \\
& \leq C\left(\sup _{t \in[0, T]}\left\|\tilde{u}_{t}(\cdot, t)\right\|_{L^{2}\left(R^{+}\right)}+\sup _{t \in[0, T]}\|\tilde{u}(\cdot, t)\|_{L^{2}\left(R^{+}\right)}\right) .
\end{aligned}
$$

Moreover, by a similar argument, we have

$$
\begin{aligned}
& \|\xi \hat{U}(\xi, \tau)\|_{L^{2}(R \times R)} \leq C\left\|U_{x}(x, t)\right\|_{L^{2}\left(R^{\times} R^{+}\right)} \\
& \leq C\left(\sup _{t \in[0, T]}\left\|\tilde{u}_{x}(\cdot, t)\right\|_{L^{2}\left(R^{+}\right)}+\sup _{t \in[0, T]}\|\tilde{u}(\cdot, t)\|_{L^{2}\left(R^{+}\right)}\right) .
\end{aligned}
$$

Thus, for $-\frac{3}{4}<s \leq-\frac{1}{2}$,

$$
\begin{aligned}
& N_{s, \frac{1}{2}}(U) \leq C \int_{-\infty}^{\infty} \int_{-\infty}^{\infty}\left[1+|\tau|+(1+|\xi|)^{3+2 s}\right]|\hat{U}(\xi, \tau)|^{2} d \xi d \tau \\
\leq & C\left(\sup _{t \in[0, T]}\left\|\tilde{u}_{t}(\cdot, t)\right\|_{L^{2}\left(R^{+}\right)}+\sup _{t \in[0, T]}\left\|\tilde{u}_{x}(\cdot, t)\right\|_{L^{2}\left(R^{+}\right)}+\sup _{t \in[0, T]}\|\tilde{u}(\cdot, t)\|_{L^{2}\left(R^{+}\right)}\right) .
\end{aligned}
$$

It is deduced that $U(x, t) \in \mathcal{X}_{s, \frac{1}{2}}^{\alpha}\left(R^{+} \times(0, T)\right)$ for any $s$ with $-\frac{3}{4}<s<-\frac{1}{2}$, and so $U$ restricted to $\left(0, \frac{T}{2}\right]$ lies in $\mathcal{X}_{s, \frac{1}{2}}^{\alpha}\left(R^{+} \times\left(0, \frac{T}{2}\right)\right)$. However, the restriction of $U(x, t)$ 
for $(x, t)$ in $R^{+} \times\left[0, T_{s}\right]$ is $\tilde{u}(x, t)$, and thus $\tilde{u}$ lies in $\mathcal{X}_{s, \frac{1}{2}}^{\alpha}\left(R^{+} \times\left(0, T_{s}\right)\right)$. Therefore, $u_{s}(x, t)$ lies in $C\left(\left[0, T_{s}\right] ; H^{3}\left(R^{+}\right)\right)$and the proof is complete.

Corollary 4.14. Let $s$ be fixed in the range $-\frac{3}{4}<s<1$. For given $\phi \in$ $H^{s}\left(R^{+}\right)$and $h \in H_{l o c}^{\frac{s+1}{3}}\left(R^{+}\right)$satisfying $\phi(0)=h(0)$ if $s>\frac{1}{2}$, the solution $u \in$ $C\left([0, T] ; H^{s}\left(R^{+}\right)\right)$given by Theorem 4.1 is a mild solution.

Proof: Choose a sequence $\left\{\phi_{n}\right\}$ in $H^{3}\left(R^{+}\right)$and a sequence $\left\{h_{n}\right\}$ in $H^{\frac{4}{3}}\left(R^{+}\right)$ such that $\phi_{n}(0)=h_{n}(0)$ for $n=1,2, \cdots, \phi_{n}$ converges to $\phi$ in the space $H^{s}\left(R^{+}\right)$ and $h_{n}$ converges to $h$ in $H^{\frac{s+1}{3}}(0, T)$. Let $u_{n}$ be the solution of (4.1) corresponding to $\phi_{n}$ and $h_{n}$ determined by Theorem 4.1. It is elementary that $u_{n}$ depends continuously on $\left(\phi_{n}, h_{n}\right)$ because it is obtained via the contraction mapping principle. Thus, as $n \rightarrow \infty, u_{n}$ converges to $u$ in $C\left([0, T] ; H^{s}\left(R^{+}\right)\right)$. By Proposition 4.13, $u_{n} \in C\left([0, T] ; H^{3}\left(R^{+}\right)\right)$is a strong solution of $(1.1)$ for $n=1,2, \cdots$. Thus, by definition, $u$ is a mild solution.

Remark: In the same manner, one can show that the solutions given by Theorem $B$ and Theorem D in Section 1 are all mild solutions.

Proposition 4.15 (uniqueness of mild solution). Let $s>-\frac{3}{4}$ be given. For any $\phi \in H^{s}\left(R^{+}\right)$and $h \in H_{\text {loc }}^{\frac{s+1}{3}}\left(R^{+}\right)$, the IBVP (4.1) admits at most one mild solution defined on the time interval $[0, T]$.

Proof: It suffices to show uniqueness in case of $s \leq \frac{3}{2}$ as the case $s>\frac{3}{2}$ follows from a straightforward energy inequality and Gronwall's Lemma. Suppose $u$ and $v$ are two mild solutions of (4.1) corresponding to the given $\phi$ and $h$. By definition, there exist two sequences $\left\{u_{n}\right\}$ and $\left\{v_{n}\right\}$ in the space $C\left([0, T] ; H^{3}\left(R^{+}\right)\right)$with

$$
u_{n}(x, 0)=\phi_{n}(x), v_{n}(x, 0)=\psi_{n}(x), u_{n}(0, t)=h_{n}(t), v_{n}(0, t)=g_{n}(t)
$$

for $n=1,2, \cdots$, such that

$$
\lim _{n \rightarrow \infty}\left\|u_{n}-u\right\|_{C\left([0, T] ; H^{s}\left(R^{+}\right)\right)}=\lim _{n \rightarrow \infty}\left\|v_{n}-v\right\|_{C\left([0, T] ; H^{s}\left(R^{+}\right)\right)}=0
$$

and $u_{n}$ and $v_{n}$ are strong solutions of (4.1) for $n=1,2, \cdots$. Define

$$
u_{m}^{*}= \begin{cases}u_{2 n} & \text { if } m=2 n^{\prime} \\ v_{2 n+1} & \text { if } m=2 n+1\end{cases}
$$

and

$$
u_{m}^{*}(x, 0)=\phi_{m}^{*}(x), u_{m}^{*}(0, t)=h_{m}^{*}(t)
$$

for $m=1,2, \cdots$. Note that $\left(\phi_{m}^{*}, h_{m}^{*}\right)$ is a Cauchy sequence in the space $H^{s}\left(R^{+}\right) \times$ $H^{\frac{s+1}{3}}(0, T)$ since both $g_{n}$ and $h_{n}$ converge to $h$ in $H^{\frac{s+1}{3}}(0, T)$ and both $\phi_{n}$ and $\psi_{n}$ converges to $\phi$ in $H^{s}\left(R^{+}\right)$. On the other hand, because of the property of persistence of regularity, $u_{m}^{*}$ is the restriction of $w_{m}$ to the domain $R^{+} \times[0, T]$ where $w_{m}$ is the solution with auxiliary data $\left(\phi_{m}^{*}, h_{m}^{*}\right)$ determined by Theorem 4.1 corresponding to $\phi_{m}^{*}$ and $h_{m}^{*}$, for $m=1,2, \cdots$. By the continuous dependence portion of Theorem $4.1,\left\{w_{m}\right\}$ is also a Cauchy sequence in the space $C\left([0, T] ; H^{s}\left(R^{+}\right)\right)$. Consequently, $u_{m}^{*}$ is a Cauchy sequence in $C\left([0, T] ; H^{s}\left(R^{+}\right)\right)$, which implies that

$$
u=v \text { in the space } C\left([0, T] ; H^{s}\left(R^{+}\right)\right) .
$$

The proof is complete. 
As a consequence of the above results, the following unconditional well-posedness theorem emerges for the IBVP (4.1)

ThEOREM 4.16 (unconditional well-posedness). For any given $s>-\frac{3}{4}$ and $r>0$, there exists a $T>0$ depending only on $r$ and $s$ such that if $\phi \in H^{s}\left(R^{+}\right)$and $h \in H_{l o c}^{\frac{s+1}{3}}\left(R^{+}\right)$satisfying the s-compatibility conditions (cf. [4]) and

$$
\|\phi\|_{H^{s}\left(R^{+}\right)}+\|h\|_{H^{\frac{s+1}{3}}(0, T)} \leq r
$$

then (4.1) admits a unique mild solution $u \in C\left(0, T ; H^{s}\left(R^{+}\right)\right)$. Moreover, the solution $u$ depends continuously on $\phi$ and $h$ in the respective spaces.

Remark : In fact, it can be further demonstrated that the solution $u$ depends on $\phi$ and $h$ analytically in the respective spaces (cf. $[\mathbf{4 4}, \mathbf{4 5}])$.

Finally, we turn to the issue of global well-posedness of the IBVP (4.1). A preliminary global result is first derived; this result will be extended by interpolation.

Proposition 4.17. Let $T>0$ and $\epsilon>0$ be given. For any $\phi \in L^{2}\left(R^{+}\right)$and $h \epsilon$ $H^{\frac{1+\epsilon}{3}}(0, T)$, the $I B V P$ (4.1) admits a unique mild solution $u \in C\left([0, T] ; L^{2}\left(R^{+}\right)\right)$. The solution $u$ has the additional property that there exists a $u^{*} \in \mathcal{X}_{0, b}$ for some $b<\frac{1}{2}$ such that

$$
\left\|u^{*}\right\|_{\mathcal{X}_{0, b}} \leq \alpha_{0}\left(\|(\phi, h)\|_{L^{2}\left(R^{+}\right) \times H^{\frac{1+\epsilon}{3}}(0, T)}\right)
$$

and $u$ is the restriction of $u^{*}$ to the domain $R^{+} \times[0, T)$. Here $\alpha_{0}: R^{+} \rightarrow R^{+}$is a nondecreasing continuous function. Moreover, the solution map

$$
u=K(\phi, h)
$$

is real analytic from the space $L^{2}\left(R^{+}\right) \times H^{\frac{1+\epsilon}{3}}(0, T)$ to $C\left([0, T] ; L^{2}\left(R^{+}\right)\right)$.

Proof: Since local well-posedness has been established in Theorem 4.16, it suffices to prove the following a priori estimate for smooth solutions $u$ of the IBVP (4.1):

$$
\sup _{0 \leq t \leq T}\|u(\cdot, t)\|_{L^{2}\left(R^{+}\right)} \leq \alpha_{T}\left(\|\phi\|_{L^{2}\left(R^{+}\right)}+\|h\|_{H^{\frac{1+\epsilon}{3}(0, T)}}\right)
$$

for the given value of $T$, where $\alpha_{T}: R^{+} \rightarrow R^{+}$is a non-decreasing function depending only on $T$.

To prove (4.23), write a smooth solution $u$ of (4.1) in the form $u=w+v$ where $v$ solves

and $w$ solves

$$
\left.\begin{array}{l}
v_{t}+v_{x}+v_{x x x}=0, \quad \text { for } x, t \geq 0, \\
v(x, 0)=0, \quad v(0, t)=h(t)
\end{array}\right\}
$$

$$
w_{t}+w_{x}+w w_{x}+(v w)_{x}+w_{x x x}=-v v_{x}
$$

with the auxiliary conditions

$$
w(x, 0)=\phi(x) \quad \text { and } \quad w(0, t)=0 .
$$


By Theorem 3.9,

$$
\sup _{x \in R^{+}}\left\|v_{x}(x, \cdot)\right\|_{L^{2}(0, T)} \leq C\|h\|_{H^{\frac{1+\epsilon}{3}}(0, T)} .
$$

Multiplying both sides of $(4.24)$ by $2 w$, integrating with respect to $x$ over $(0, \infty)$, and integrating by parts appropriately, there obtains (4.27)

$\frac{d}{d t} \int_{0}^{\infty} w^{2}(x, t) d x+w_{x}^{2}(0, t)=-2 \int_{0}^{\infty} v(x, t) v_{x}(x, t) w(x, t) d x-\int_{0}^{\infty} v_{x}(x, t) w^{2}(x, t) d x$ for any $t \in(0, T)$. In consequence, it is deduced that

$$
\begin{aligned}
& \frac{d}{d t}\|w(\cdot, t)\|_{L^{2}\left(R^{+}\right)}^{2} \leq \int_{0}^{\infty}\left(2\left|v(x, t) v_{x}(x, t) w(x, t)\right|+\left|v_{x}(x, t) w^{2}(x, t)\right|\right) d x \\
& \leq\|v(\cdot, t)\|_{H^{1}\left(R^{+}\right)}^{2}\|w(\cdot, t)\|_{L^{2}\left(R^{+}\right)}+\sup _{x \in R^{+}}\left|v_{x}(\cdot, t)\right|\|w(\cdot, t)\|_{L^{2}\left(R^{+}\right)}^{2},
\end{aligned}
$$

from which it follows that

$$
\frac{d}{d t}\|w(\cdot, t)\|_{L^{2}\left(R^{+}\right)} \leq\|v(\cdot, t)\|_{H^{1}\left(R^{+}\right)}^{2}+\sup _{x \in R^{+}}\left|v_{x}(\cdot, t)\right|\|w(\cdot, t)\|_{L^{2}\left(R^{+}\right)}
$$

for $t \in(0, T)$. The inequality (4.23) then follows from Gronwall's Lemma and (4.26). The proof is completed by approximating the rough solution by a sequence of smooth solutions and noting that (4.27) continues to hold in the limit.

THEOREM 4.18. For any $\epsilon>0$, the IBVP (4.1) is globally unconditionally well-posed in the space

$$
H^{s}\left(R^{+}\right) \times H_{l o c}^{\frac{1+s+\epsilon}{3}}\left(R^{+}\right)
$$

if $0 \leq s<3$ and is globally unconditionally well-posed in the space

$$
H^{s}\left(R^{+}\right) \times H_{l o c}^{\frac{1+s}{3}}\left(R^{+}\right)
$$

for $s \geq 3$.

Proof: The IBVP (4.1) is known from the results in [4] to be unconditionally globally well-posed in the space $H^{s}\left(R^{+}\right) \times H_{l o c}^{\frac{1+s}{3}}\left(R^{+}\right)$for $s \geq 3$. The case of $0<s<3$ follows from this result and Proposition 4.17 by nonlinear interpolation theory $[\mathbf{4 1}, \mathbf{3}]$ as expounded in $[\mathbf{4}]$. The proof is complete.

\section{Appendices}

5.1. Appendix I. Proof of Lemma 4.2: Let $u=W_{R}(t) \phi$. Then the same proof as presented in $[\mathbf{3 3}]$ demonstrates that

$$
\Lambda_{s, b}\left(\psi\left(\delta^{-1} t\right) u\right) \leq C \delta^{\frac{1-2 b}{2}}\|\phi\|_{H^{s}\left(R^{+}\right)}
$$

for some constant $C$ independent of $\delta$ and $\phi$. (Note that it is required in $[\mathbf{3 3}]$ that $b>\frac{1}{2}$, which is, in fact, not needed in the proof.) Since

$$
\lambda_{\alpha}\left(\psi\left(\delta^{-1} t\right) u\right) \leq C \Lambda_{s, \alpha}\left(\psi\left(\delta^{-1} t\right) u\right)
$$

it follows from (5.1) that

$$
\lambda_{\alpha}\left(\psi\left(\delta^{-1} t\right) u\right) \leq C \delta^{\frac{1-2 \alpha}{2}}\|\phi\|_{H^{s}\left(R^{+}\right)} .
$$

Estimate (4.2) is then established by combining (5.1) and (5.2). 
According to $[33]$,

$$
\Lambda_{s, b}\left(\psi\left(\delta^{-1} t\right) v\right) \leq C \delta^{\frac{1-2 b}{2}} \Lambda_{s, b}(v)
$$

where $b$ is required to be strictly greater than $\frac{1}{2}$. However, if $b \leq \frac{1}{2}$, then the proof of (5.3) in [33] is straightforwardly modified (cf. [46]) to obtain the estimate

$$
\Lambda_{s, b}\left(\psi\left(\delta^{-1} t\right) v\right) \leq C \delta^{\frac{1-2 b^{\prime}}{2}} \Lambda_{s, b}(v)
$$

where $b^{\prime}$ is any number strictly greater than $1 / 2$. Combining estimate (5.4) with the estimate

$$
\lambda_{\alpha}\left(\psi\left(\delta^{-1} t\right) v\right) \leq C \delta^{\frac{1-2 \alpha}{2}} \lambda_{\alpha}(v)
$$

which follows from exactly the same observations as did (5.3), yields (4.3).

The proof of $(4.4)$ is based on analysis in $[\mathbf{1 1}, \mathbf{3 3}]$, though with a little modification. First observe that

$$
\begin{gathered}
\psi\left(\delta^{-1} t\right) \int_{0}^{t} W_{R}\left(t-t^{\prime}\right) f\left(t^{\prime}\right) d t^{\prime} \\
=\psi\left(\delta^{-1} t\right) \int_{-\infty}^{\infty} \int_{-\infty}^{\infty} e^{i x \xi} \hat{f}(\xi, \tau) \psi\left(\tau-\left(\xi^{3}-\xi\right)\right) \frac{e^{i \tau t}-e^{i t\left(\xi^{3}-\xi\right)}}{\tau-\left(\xi^{3}-\xi\right)} d \xi d \tau \\
\quad+\psi\left(\delta^{-1} t\right) \int_{-\infty}^{\infty} \int_{-\infty}^{\infty} e^{i x \xi} \hat{f}(\xi, \tau)\left(1-\psi\left(\tau-\left(\xi^{3}-\xi\right)\right)\right) \frac{e^{i \tau t}-e^{i t\left(\xi^{3}-\xi\right)}}{\tau-\left(\xi^{3}-\xi\right)} d \xi d \tau \\
:=A+B .
\end{gathered}
$$

By a Taylor series expansion,

$$
A=\sum_{k=1}^{\infty} \frac{i^{k}}{k !} \delta^{k} \psi_{k}\left(\delta^{-1} t\right) W_{R}(t) g
$$

with

$$
\hat{g}(\xi)=\int_{-\infty}^{\infty} \hat{f}(\xi, \tau)\left(\tau-\left(\xi^{3}-\xi\right)\right)^{k-1} \psi\left(\tau-\left(\xi^{3}-\xi\right)\right) d \tau
$$

and

$$
\psi_{k}\left(\delta^{-1} t\right):=\left(\frac{t}{\delta}\right)^{k} \psi\left(\delta^{-1} t\right), \quad k=1,2, \cdots
$$

As $\delta \leq 1$ and $b \leq 1$,

$$
\delta^{2} \int_{-\infty}^{\infty}\left|\hat{\psi}_{k}(\delta \tau)\right|^{2}\left(1+|\tau|^{2 b}\right) d \tau \leq C \delta^{1-2 b}(k+1)^{2} .
$$


Moreover, because of the restriction on the support of $\psi$,

$$
\begin{aligned}
\|g\|_{H^{s}(R)}^{2} & \leq \\
C \int_{-\infty}^{\infty}(1+|\xi|)^{2 s}\left|\int_{-\infty}^{\infty} \hat{f}(\xi, \tau)\left(\tau-\left(\xi^{3}-\xi\right)\right)^{k-1} \psi\left(\tau-\left(\xi^{3}-\xi\right)\right) d \tau\right|^{2} d \xi & \left.\hat{f}(\xi, \tau) d \tau\right|^{2} d \xi \\
& \leq C \int_{-\infty}^{\infty}(1+|\xi|)^{2 s} \mid \int_{\left|\tau-\left(\xi^{3}-\xi\right)\right|<1} \\
& \leq C \int_{-\infty}^{\infty}(1+|\xi|)^{2 s}\left|\int_{-\infty}^{\infty} \frac{\hat{f}(\xi, \tau)}{1+\left|\tau-\left(\xi^{3}-\xi\right)\right|} d \tau\right|^{2} d \xi
\end{aligned}
$$

Thus, applying (4.2) gives

$$
\begin{aligned}
\|A\|_{X_{s, b}^{\alpha}} & \leq C \sum_{k=1}^{\infty} \frac{\delta^{k}(k+1)^{2}}{k !} \delta^{\frac{1-2 b^{*}}{2}}\|g\|_{H^{s}(R)} \\
& \leq C \delta^{\frac{1-2 b^{*}}{2}}\left(\left(\int_{-\infty}^{\infty}(1+|\xi|)^{2 s}\left|\int_{-\infty}^{\infty} \frac{\hat{f}(\xi, \tau)}{1+\left|\tau-\left(\xi^{3}-\xi\right)\right|} d \tau\right|^{2} d \xi\right)^{\frac{1}{2}} .\right.
\end{aligned}
$$

Next, consider the term $B$ which can be written as

$$
B=B_{1}+B_{2}
$$

with

$$
B_{1}=-\psi\left(\delta^{-1} t\right) \int_{-\infty}^{\infty} e^{i\left(x \xi+t\left(\xi^{3}-\xi\right)\right)}\left(\int_{-\infty}^{\infty} \frac{1-\psi\left(\tau-\left(\xi^{3}-\xi\right)\right)}{\tau-\left(\xi^{3}-\xi\right)} \hat{f}(\xi, \tau)\right) d \xi
$$

and

$$
B_{2}=\psi\left(\delta^{-1} t\right) \int_{-\infty}^{\infty} e^{i(x \xi+t \tau)}\left(\int_{-\infty}^{\infty} \frac{1-\psi\left(\tau-\left(\xi^{3}-\xi\right)\right)}{\tau-\left(\xi^{3}-\xi\right)} \hat{f}(\xi, \tau)\right) d \xi .
$$

For $B_{1},(4.2)$ yields

$$
\left\|B_{1}\right\|_{X_{s, b}^{\alpha}}^{2} \leq C \delta^{1-2 b^{*}} \int_{-\infty}^{\infty}(1+|\xi|)^{2 s}\left(\int_{-\infty}^{\infty} \frac{|\hat{f}(\xi, \tau)|}{\left(1+\left|\tau-\left(\xi^{3}-\xi\right)\right|\right.} d \tau\right)^{2} d \xi .
$$

As for $B_{2}$, applying (4.3) leads to

$$
\begin{aligned}
\left\|B_{2}\right\|_{X_{s, b}^{\alpha}}^{2} \leq C & \delta^{1-2 b^{*}}\left\|\int_{-\infty}^{\infty} e^{i(x \xi+t \tau)}\left(\int_{-\infty}^{\infty} \frac{1-\psi\left(\tau-\left(\xi^{3}-\xi\right)\right)}{\tau-\left(\xi^{3}-\xi\right)} \hat{f}(\xi, \tau)\right) d \xi\right\|_{X_{s, b}^{\alpha}}^{2} \\
\leq C & \delta^{1-2 b^{*}} \int_{-\infty}^{\infty} \int_{-\infty}^{\infty}(1+|\xi|)^{2 s} \frac{|\hat{f}(\xi, \tau)|^{2}}{\left(1+\left|\tau-\left(\xi^{3}-\xi\right)\right|\right)^{2(1-b)}} d \xi d \tau \\
& +C \delta^{1-2 b^{*}} \int_{-\infty}^{\infty} \int_{|\xi| \leq 1} \frac{|\hat{f}(\xi, \tau)|^{2}}{(1+|\tau|)^{2(1-\alpha)}} d \xi d \tau
\end{aligned}
$$

The proof is complete.

\section{Proof of Lemma 4.3.}


(a). Estimate (4.5) was established in [4], Lemma 3.10 when $s \geq 0$. The proof presented there is also valid for $s \leq 0$.

(b). Let $u=W_{R}(t) \phi$ and $s_{1}:=\frac{s+1}{3}$. Then $s_{1} \geq 0$ when $-1 \leq s<\infty$. Observe that there is a constant $C$ depending only on $s$ such that

$$
\|\psi u\|_{H_{t}^{s_{1}(R)}} \leq C\left(\|\psi u\|_{L_{t}^{2}(R)}+\left\|D_{t}^{s_{1}}(\psi u)\right\|_{L_{t}^{2}(R)}\right) .
$$

For $0 \leq s_{1}<1$, the Leibniz rule for fractional derivative implies

$$
\begin{aligned}
& \left\|D_{t}^{s_{1}}(\psi(t) u(x, t))-\psi(t) D_{t}^{s_{1}} u(x, t)-D_{t}^{s_{1}} \psi(t) u(x, t)\right\|_{L_{t}^{2}(R)} \\
& \leq C\|\psi\|_{L^{\infty}(R)}\left\|D_{t}^{s_{1}} u(x, t)\right\|_{L_{t}^{2}(R)} .
\end{aligned}
$$

Thus, it transpires that

$$
\|\psi(t) u(x, t)\|_{H_{t}^{s_{1}(R)}} \leq C\|u(x, t)\|_{H_{t}^{s_{1}}(R)}
$$

where $C$ depends on $s$ and $\psi$. Estimate (4.6) then follows from (4.5). If $1 \leq s_{1}<2$, write $s_{1}$ as $s_{1}=1+s^{\prime}$ with $0 \leq s^{\prime}<1$; with this notation, we have

$$
D_{t}^{s_{1}}(\psi(t) u(x, t))=D_{t}^{s^{\prime}}\left(\psi^{\prime}(t) u(x, t)+\psi(t) \partial_{t} u(x, t)\right)
$$

It follows that

$$
\begin{aligned}
& \left\|D_{t}^{s_{1}}(\psi(t) u(x, t))\right\|_{L_{t}^{2}(R)} \\
& \leq C\left(\left\|D_{t}^{s^{\prime}}\left(\psi^{\prime}(t) u(x, t)\right)\right\|_{L_{t}^{2}(R)}+\left\|D_{t}^{s^{\prime}}\left(\psi(t) \partial_{t} u(x, t)\right)\right\|_{L_{t}^{2}(R)}\right) \\
& \leq C\left(\|u(x, t)\|_{H_{t}^{s^{\prime}(R)}}+\|u(x, t)\|_{H_{t}^{s_{1}}(R)}\right) \\
& \leq C\|\phi\|_{H^{s}(R)} .
\end{aligned}
$$

One shows by continuing this bootstrapping argument that (4.6) holds for any $s_{1} \geq 2$. The proof is complete.

Proof of Lemma 4.4. First assume that $-1 \leq s \leq 2-3 b$. As in the proof of Lemma 4.2 , write $w(x, t)$ as

$$
w(x, t)=A(x, t)+B_{1}(x, t)+B_{2}(x, t)
$$

where

$$
\begin{gathered}
A(x, t)=\sum_{k=1}^{\infty} \frac{i^{k}}{k !} t^{k} \psi(t) W_{R}(t) g, \\
B_{1}(x, t)=\psi(t) W_{R}(t) q,
\end{gathered}
$$

and $B_{2}(x, t)$ is the remainder. Then, Lemma 4.3 yields

$$
\begin{aligned}
& \sup _{x \in R}\|A(x, t)\|_{H_{t}^{\frac{s+1}{2}}(R)}^{2} \leq C\|g\|_{H^{s}(R)}^{2} \\
& \leq C \int_{-\infty}^{\infty}(1+|\xi|)^{2 s}\left|\int_{-\infty}^{\infty} \frac{\hat{f}(\xi, \tau)}{1+\left|\tau-\left(\xi^{3}-\xi\right)\right|} d \tau\right|^{2} d \xi .
\end{aligned}
$$


and

$$
\begin{aligned}
& \sup _{x \in R}\left\|B_{1}(x, t)\right\|_{H_{t}^{\frac{s+1}{2}}(R)}^{2} \leq C\|q\|_{H^{s}(R)}^{2} \\
& \leq C \int_{-\infty}^{\infty}(1+|\xi|)^{2 s}\left(\int_{-\infty}^{\infty} \frac{|\hat{f}(\xi, \tau)|}{\left(1+\left|\tau-\left(\xi^{3}-\xi\right)\right|\right.} d \tau\right)^{2} d \xi .
\end{aligned}
$$

As for $B_{2}(x, t)$, notice that

$$
\begin{gathered}
\sup _{x \in R}\left\|B_{2}(x, t)\right\|_{H_{t}^{\frac{s+1}{3}}(R)} \\
\leq C \int_{-\infty}^{\infty}(1+|\tau|)^{\frac{2(s+1)}{3}}\left(\int_{-\infty}^{\infty} \frac{\left|1-\psi\left(\tau-\left(\xi^{3}-\xi\right)\right)\right|}{\left|\tau-\left(\xi^{3}-\xi\right)\right|} \hat{f}(\xi, \tau) d \xi\right)^{2} d \tau \\
\leq C \int_{-\infty}^{\infty}(1+|\tau|)^{\frac{2(s+1)}{3}}\left(\int_{-\infty}^{\infty} \frac{1}{1+\left|\tau-\left(\xi^{3}-\xi\right)\right|} \hat{f}(\xi, \tau) d \xi\right)^{2} d \tau \\
\leq C \int_{-\infty}^{\infty}(1+|\tau|)^{\frac{2(s+1)}{3}} G(\tau) \int_{-\infty}^{\infty} \frac{|\hat{f}(\xi, \tau)|^{2}(1+|\xi|)^{2 s}}{\left(1+\left|\tau-\left(\xi^{3}-\xi\right)\right|\right)^{2 b}} d \xi d \tau
\end{gathered}
$$

where

$$
\begin{aligned}
G(\tau) & :=\int_{-\infty}^{\infty} \frac{d \xi}{(1+|\xi|)^{2 s}\left(1+\left|\tau-\left(\xi^{3}-\xi\right)\right|\right)^{2(1-b)}} \\
& \leq C \int_{-\infty}^{\infty} \frac{d \eta}{|\eta|^{\frac{2}{3}}(1+|\eta|)^{\frac{2 s}{3}}(1+|\tau-\eta|)^{2(1-b)}} .
\end{aligned}
$$

Therefore, it suffices to show that there exists a constant $C$ such that for any $\tau \in R$,

$$
G(\tau) \leq \frac{C}{(1+|\tau|)^{\frac{2(s+1)}{3}}} .
$$

Write $G$ in the form

$$
G(\tau)=G_{1}(\tau)+G_{2}(\tau)+G_{3}(\tau)
$$

with

and

$$
\begin{gathered}
G_{1}(\tau)=\int_{|\eta| \leq \frac{1}{2}|\tau|} \frac{d \eta}{|\eta|^{\frac{2}{3}}(1+|\eta|)^{\frac{2 s}{3}}(1+|\tau-\eta|)^{2(1-b)}}, \\
G_{2}(\tau)=\int_{\frac{1}{2}|\tau|<|\eta|<2|\tau|} \frac{d \eta}{|\eta|^{\frac{2}{3}}(1+|\eta|)^{\frac{2 s}{3}}(1+|\tau-\eta|)^{2(1-b)}}
\end{gathered}
$$

$$
G_{3}(\tau)=\int_{2|\tau| \leq|\eta|} \frac{d \eta}{|\eta|^{\frac{2}{3}}(1+|\eta|)^{\frac{2 s}{3}}(1+|\tau-\eta|)^{2(1-b)}} .
$$

In the region $|\eta| \leq \frac{|\tau|}{2}$, note that $(1+|\tau-\eta|) \sim(1+|\tau|)$ and $|\tau-\eta|>|\eta|$. Thus, for $s \leq 0$,

$$
\begin{aligned}
G_{1}(\tau) & \leq C(1+|\tau|)^{-\frac{2(s+1)}{3}} \int_{-\infty}^{\infty} \frac{d \eta}{|\eta|^{\frac{2}{3}(1+|\eta|)^{2(1-b)-\frac{2}{3}}}} \\
\leq & \frac{C}{(1+|\tau|)^{\frac{2(s+1)}{3}}} .
\end{aligned}
$$


as $b<\frac{1}{2}$. When $0 \leq s \leq 2-3 b$,

$$
\begin{aligned}
& G_{1}(\tau) \leq C(1+|\tau|)^{-\frac{2(s+1)}{3}} \int_{-\infty}^{\infty} \frac{d \eta}{|\eta|^{\frac{2}{3}}(1+|\eta|)^{\frac{2 s}{3}}(1+|\tau-\eta|)^{2(1-b)-\frac{2(s+1)}{3}}} \\
& \leq C(1+|\tau|)^{-\frac{2(s+1)}{3}} \int_{-\infty}^{\infty} \frac{d \eta}{|\eta|^{\frac{2}{3}}(1+|\eta|)^{2(1-b)-\frac{2}{3}}} \\
& \quad \leq \frac{C}{(1+|\tau|)^{\frac{2(s+1)}{3}}}
\end{aligned}
$$

In the region $\frac{|\tau|}{2}<|\eta|<2|\tau|$,

$$
\begin{aligned}
G_{2}(\tau) & \leq C(1+|\tau|)^{-\frac{2(s+1)}{3}} \int_{\frac{|\tau|}{2}}^{2|\tau|} \frac{d \eta}{(1+|\tau-\eta|)^{2(1-b)}} \\
\leq & \frac{C}{(1+|\tau|)^{\frac{2(s+1)}{3}}}
\end{aligned}
$$

since $b<\frac{1}{2}$. In the region $2|\tau| \leq|\eta|$, it is the case that $1+|\tau-\eta|=O(1+|\eta|)$. Thus

$$
\begin{aligned}
G_{3}(\tau) & \leq C \int_{|\eta| \geq 2|\tau|} \frac{d \eta}{|\eta|^{\frac{2(s+1)}{3}}(1+|\eta|)^{2(1-b)}} \\
\leq & C(1+|\tau|)^{-\frac{2(s+1)}{3}} .
\end{aligned}
$$

We have thus proved (4.7) for $-1 \leq s \leq 2-3 b$. In particular, it holds for $s=-1$, which is to say

$$
\sup _{x \in R}\|\psi(t) w(x, t)\|_{L_{t}^{2}(R)} \leq C\|f\|_{Y_{-1, b}^{0}} .
$$

To see (4.7) is valid for other values of $s$, let $v=w_{x x x}$. The definition of $w$ implies that

$$
v(x, t)=\int_{0}^{t}\left[W_{R}\left(t-t^{\prime}\right) f_{x x x}\left(\cdot, t^{\prime}\right)\right](x) d t^{\prime} .
$$

Using the estimate (4.7) just proved for $-1 \leq s \leq 2-3 b$, it is deduced that

$$
\sup _{x \in R}\|\psi(t) v(x, t)\|_{H_{t}^{\frac{s+1}{3}}(R)} \leq C\|f\|_{Y_{s+3, b}^{0}}
$$

for $-1 \leq s \leq 2-3 b$. Since $w_{t}=f-w_{x x x}-w_{x}$, one sees that

$$
\sup _{x \in R}\left\|\psi(t) w_{t}(x, t)\right\|_{H_{t}^{\frac{s+1}{3}}(R)} \leq C\|f\|_{Y_{s+3, b}^{0}},
$$

or, equivalently,

$$
\sup _{x \in R}\|\psi(t) w(x, t)\|_{H_{t}^{\frac{s+4}{3}}(R)} \leq C\|f\|_{Y_{s+3, b}^{0}} .
$$

In particular,

$$
\sup _{x \in R}\|\psi(t) w(x, t)\|_{H_{t}^{1}(R)} \leq C\|f\|_{Y_{2, b}^{0}} .
$$

By standard interpolation, (4.7) holds for $-1 \leq s \leq 2$. A continuation of the bootstrap argument just applied yields the desired result for other values of $s$. This completes the proof. 
Proof of Lemma 4.6. The proof is based on some minor modifications of arguments in $[\mathbf{1 1}, \mathbf{3 3}]$. By the definition of the space $Y_{s, b_{1}}^{\alpha}$ it suffices to establish the following three estimates:

$$
\begin{gathered}
\mathcal{Q}_{-s, b_{1}}(w) \leq C \delta^{\theta_{0}}\|u\|_{X_{-s, b}^{\alpha}}\|v\|_{X_{-s, b}^{\alpha}}, \\
\mathcal{P}_{\alpha}(w) \leq C \delta^{\theta_{0}}\|u\|_{X_{-s, b}^{\alpha}}\|v\|_{X_{-s, b}^{\alpha}}
\end{gathered}
$$

and

$$
\mathcal{G}(w) \leq C \delta^{\theta_{0}}\|u\|_{X_{-s, b}}\|v\|_{X_{-s, b}^{\alpha}} .
$$

Define

$$
p(x, t)=\sigma\left(\delta^{-1} t\right) u(x, t), \quad q(x, t)=\sigma\left(\delta^{-1} t\right) v(x, t),
$$

where $\sigma$ is as before, and let $w$ be defined via its Fourier transform, viz.

$$
\hat{w}(\xi, \lambda)=c \xi \hat{q} * \hat{p}
$$

Also define

$$
\beta_{b, \alpha}(\xi, \lambda)=\left(1+\left|\lambda-\left(\xi^{3}-\xi\right)\right|\right)^{b}+\chi(\xi)(1+|\lambda|)^{\alpha}
$$

where $\chi=\chi_{[-1,1]}$ and

$$
f(\xi, \lambda)=(1+|\xi|)^{-s} \beta_{b, \alpha}(\xi, \lambda)|\hat{p}(\xi, \lambda)|, \quad g(\xi, \lambda)=(1+|\xi|)^{-s} \beta_{b, \alpha}(\xi, \lambda)|\hat{q}(\xi, \lambda)| .
$$

It follows that

$$
\|f\|_{L_{\xi}^{2} L_{\tau}^{2}}=\|p\|_{X_{-s, b}} \quad \text { and } \quad\|g\|_{L_{\xi}^{2} L_{\tau}^{2}}=\|q\|_{X_{-s, b}} .
$$

Moreover, there is a constant $C$ for which

$|\hat{w}(\xi, \lambda)| \leq C|\xi| \int_{-\infty}^{\infty} \int_{-\infty}^{\infty} \frac{\left(1+\left|\xi_{1}\right|\right)^{s} f\left(\xi_{1}, \lambda_{1}\right)}{\beta_{b, \alpha}\left(\xi_{1}, \lambda_{1}\right)} \frac{\left(1+\left|\xi-\xi_{1}\right|\right)^{s} g\left(\xi-\xi_{1}, \lambda-\lambda_{1}\right)}{\beta_{b, \alpha}\left(\xi-\xi_{1}, \lambda-\lambda_{1}\right)} d \xi_{1} \lambda_{1}$.

To estimate $\Lambda_{s, b}(w)$, proceed by duality. Let $h(\xi, \lambda) \geq 0$, with $\|h\|_{L_{\xi}^{2} L_{\lambda}^{2}} \leq 1$ and consider the expression

$$
\begin{aligned}
& \Upsilon=\int_{R^{4}} \frac{|\xi| h(\xi, \lambda)}{(1+|\xi|)^{s}\left(1+\left|\lambda-\left(\xi^{3}-\xi\right)\right|\right)^{b_{1}}} \\
& \frac{\left(1+\left|\xi_{1}\right|\right)^{s} f\left(\xi_{1}, \lambda_{1}\right)}{\beta_{b, \alpha}\left(\xi_{1}, \lambda_{1}\right)} \frac{\left(1+\left|\xi-\xi_{1}\right|\right)^{s} g\left(\xi-\xi_{1}, \lambda-\lambda_{1}\right)}{\beta_{b, \alpha}\left(\xi-\xi_{1}, \lambda-\lambda_{1}\right)} d \mu
\end{aligned}
$$

where $d \mu=d \xi_{1} d \lambda_{1} d \xi d \lambda$. By symmetry, it suffices to estimate the integral in the domain

Because of the identity

$$
\left|\xi_{1}\right| \leq\left|\xi-\xi_{1}\right| .
$$

$$
\lambda-\left(\xi^{3}-\xi\right)-\left[\lambda_{1}-\left(\xi_{1}^{3}-\xi_{1}\right)+\left(\lambda-\lambda_{1}\right)-\left(\xi^{3}-\xi-\left(\xi_{1}^{3}-\xi_{1}\right)\right)\right]=3 \xi\left(\xi-\xi_{1}\right) \xi_{1},
$$

one of the following three cases always occurs: 


$$
\begin{cases}\text { (a) } & \left|\lambda-\left(\xi^{3}-\xi\right)\right| \geq|\xi|\left|\xi-\xi_{1}\right|\left|\xi_{1}\right|, \\ \text { (b) } & \left|\lambda_{1}-\left(\xi_{1}^{3}-\xi\right)\right| \geq|\xi|\left|\xi-\xi_{1}\right|\left|\xi_{1}\right|, \\ (c) & \left|\lambda-\lambda_{1}-\left(\left(\xi-\xi_{1}\right)^{3}-\left(\xi-\xi_{1}\right)\right)\right| \geq|\xi|\left|\xi-\xi_{1}\right|\left|\xi_{1}\right| .\end{cases}
$$

To bound the integral $\Upsilon$ we split the domain of integration into several pieces.

Assume first that $|\xi| \leq 2$ and $\left|\xi_{1}\right| \leq 1$. Since $\left|\xi_{1}\right| \leq\left|\xi-\xi_{1}\right|$ then $\left|\xi-\xi_{1}\right| \leq 3$, and consequently the integral $\Upsilon$ restricted to this domain is bounded as before:

$$
\begin{aligned}
\int_{R^{4}} h(\xi, \lambda) & \frac{f\left(\xi_{1}, \lambda_{1}\right)}{\left(1+\left|\lambda_{1}-\left(\xi_{1}^{3}-\xi_{1}\right)\right|\right)^{b}} \frac{g\left(\xi-\xi_{1}, \lambda-\lambda_{1}\right)}{\left(1+\left|\lambda-\lambda_{1}-\left(\left(\xi-\xi_{1}\right)^{3}-\left(\xi-\xi_{1}\right)\right)\right|\right)^{b}} d \mu \\
& =\left\langle h, \hat{F}_{b} * \hat{G}_{b}\right\rangle=\left\langle H_{0}, F_{b} G_{b}\right\rangle \\
& \leq C\|h\|_{L_{\xi}^{2} L_{\lambda}^{2}}\left\|F_{b}\right\|_{L_{x}^{4} L_{t}^{4}}\left\|G_{b}\right\|_{L_{x}^{4} L_{t}^{4}} \\
& \leq C\|h\|_{L_{\xi}^{2} L_{\lambda}^{2}}\|g\|_{L_{\xi}^{2} L_{\lambda}^{2}}\left\|F_{b}\right\|_{L_{x}^{4} L_{t}^{4}},
\end{aligned}
$$

if $b>\frac{1}{3}$, where

$$
\hat{H}_{\rho}=\frac{h(\xi, \lambda)}{\left(1+\left|\lambda-\left(\xi^{3}-\xi\right)\right|\right)^{\rho}}
$$

and $\hat{F}_{b}$ and $\hat{H}_{b}$ are defined similarly.

Assume next that $|\xi| \leq 2$ and $1 \leq\left|\xi_{1}\right| \leq\left|\xi_{1}-\xi\right|$. We consider the three cases in (5.11). If (a) holds, the integral $\Upsilon$ in this region is bounded by

$$
\begin{aligned}
& \int_{R^{4}} h(\xi, \lambda) \frac{\left|\xi_{1}\right|^{s-b_{1}} f\left(\xi_{1}, \lambda_{1}\right)}{\left(1+\left|\lambda_{1}-\left(\xi_{1}^{3}-\xi_{1}\right)\right|\right)^{b}} \frac{\left|\xi-\xi_{1}\right|^{s-b_{1}} g\left(\xi-\xi_{1}, \lambda-\lambda_{1}\right)}{\left(1+\mid\left(\lambda-\lambda_{1}-\left(\left(\xi-\xi_{1}\right)^{3}-\left(\xi-\xi_{1}\right)\right) \mid\right)^{b}\right.} d \mu \\
& \leq \int_{R^{4}} h(\xi, \lambda) \frac{\left|\xi_{1}\right|^{\frac{1}{8}} f\left(\xi_{1}, \lambda_{1}\right)}{\left(1+\left|\lambda_{1}-\left(\xi_{1}^{3}-\xi_{1}\right)\right|\right)^{b}} \frac{\left|\xi-\xi_{1}\right|^{\frac{1}{8}} g\left(\xi-\xi_{1}, \lambda-\lambda_{1}\right)}{\left(1+\mid\left(\lambda-\lambda_{1}-\left(\left(\xi-\xi_{1}\right)^{3}-\left(\xi-\xi_{1}\right)\right) \mid\right)^{b}\right.} d \mu \\
& =\left\langle h, \widehat{D_{x}^{\frac{1}{8}} F_{b}} * \widehat{D_{x}^{\frac{1}{8}} G_{b}}\right\rangle=\left\langle H_{0}, D_{x}^{\frac{1}{8}} F_{b} D_{x}^{\frac{1}{8}} G_{b}\right\rangle \\
& \leq C\|h\|_{L_{\xi}^{2} L_{\lambda}^{2}}\left\|D_{x}^{\frac{1}{8}} F_{b}\right\|_{L_{x}^{4} L_{t}^{4}}\left\|D_{x}^{\frac{1}{8}} G_{b}\right\|_{L_{x}^{4} L_{t}^{4}} \\
& \leq C\|h\|_{L_{\xi}^{2} L_{\lambda}^{2}}\|g\|_{L_{\xi}^{2} L_{\lambda}^{2}}\left\|D_{x}^{\frac{1}{8}} F_{b}\right\|_{L_{x}^{4} L_{t}^{4}} .
\end{aligned}
$$

Here, it was assumed that

$$
s-b_{1} \leq \frac{1}{8}, \quad b_{1} \leq 1, \quad b>\frac{3}{8} .
$$


If case (b) holds, the integral $\Upsilon$ is bounded in the following way:

$$
\begin{aligned}
& \int_{R^{4}} \frac{|\xi|^{1-b} \chi\left(\frac{1}{2} \xi\right) h(\xi, \lambda)}{\left(1+\left|\lambda-\left(\xi^{3}-\xi\right)\right|\right)^{b_{1}}} \frac{\left|\xi_{1}\right|^{s-b} f\left(\xi_{1}, \lambda_{1}\right)\left|\xi-\xi_{1}\right|^{s-b} g\left(\xi-\xi_{1}, \lambda-\lambda_{1}\right)}{\left(1+\left|\lambda-\lambda_{1}-\left(\left(\xi-\xi_{1}\right)^{3}-\left(\xi-\xi_{1}\right)\right)\right|\right)^{b}} d \mu \\
& =\int_{R^{4}} \frac{\chi\left(\frac{\xi}{2}\right) h(\xi, \lambda)}{\left(1+\left|\lambda-\left(\xi^{3}-\xi\right)\right|\right)^{b_{1}}} \frac{f\left(\xi_{1}, \lambda_{1}\right)\left|\xi-\xi_{1}\right|^{\frac{1}{4}} g\left(\xi-\xi_{1}, \lambda-\lambda_{1}\right)}{\left(1+\left|\lambda-\lambda_{1}-\left(\left(\xi-\xi_{1}\right)^{3}-\left(\xi-\xi_{1}\right)\right)\right|\right)^{b}} d \mu \\
& =\left\langle\widehat{\mathcal{H}_{b_{1}}}, \widehat{F_{0}} * \widehat{D_{x}^{\frac{1}{4}} G_{b}}\right\rangle=\left\langle\mathcal{H}_{b_{1}}, F_{0} D_{x}^{\frac{1}{4}} G_{b}\right\rangle \\
& \leq C\left\|\mathcal{H}_{b_{1}}\right\|_{L_{x}^{4} L_{t}^{6}}\left\|F_{0}\right\|_{L_{x}^{2} L_{t}^{2}}\left\|D_{x}^{\frac{1}{4}} G_{b}\right\|_{L_{x}^{4} L_{t}^{3}} \\
& \leq C\|h\|_{L_{\xi}^{2} L_{\lambda}^{2}}\|f\|_{L_{\xi}^{2} L_{\tau}^{2}}\left\|D_{x}^{\frac{1}{4}} G_{b}\right\|_{L_{x}^{4} L_{t}^{3}},
\end{aligned}
$$

where

$$
\hat{\mathcal{H}}_{b_{1}}(\xi, \lambda)=\frac{h(\xi, \lambda) \chi\left(\frac{1}{2} \xi\right)}{\left(1+\left|\lambda-\left(\xi^{3}-\xi\right)\right|\right)^{b_{1}}}
$$

To effect this estimate, it was assumed that

$$
s-b<\frac{1}{8}, \quad 1 \geq b>\frac{1}{3}, \quad b_{1}>\frac{5}{12} .
$$

If (c) holds, then

$$
\begin{aligned}
& \Upsilon \leq \int_{R^{4}} \frac{|\xi|^{1-b} \chi\left(\frac{1}{2} \xi\right) h(\xi, \lambda)}{\left(1+\left|\lambda-\left(\xi^{3}-\xi\right)\right|\right)^{b_{1}}} \frac{\left|\xi_{1}\right|^{\frac{1}{8}} f\left(\xi_{1}, \lambda_{1}\right)\left|\xi-\xi_{1}\right|^{\frac{1}{8}} g\left(\xi-\xi_{1}, \lambda-\lambda_{1}\right)}{\left(1+\left|\lambda_{1}-\left(\xi_{1}^{3}-\xi_{1}\right)\right|\right)^{b}} d \mu \\
& \leq C \int_{R^{4}} \frac{\chi\left(\frac{1}{2} \xi\right) h(\xi, \lambda)}{\left(1+\left|\lambda-\left(\xi^{3}-\xi\right)\right|\right)^{b_{1}}} \frac{\left|\xi_{1}\right|^{\frac{1}{8}} f\left(\xi_{1}, \lambda_{1}\right) g\left(\xi-\xi_{1}, \lambda-\lambda_{1}\right)}{\left(1+\left|\lambda_{1}-\left(\xi_{1}^{3}-\xi_{1}\right)\right|\right)^{b}} d \mu+ \\
& \quad+C \int_{R^{4}} \frac{\chi\left(\frac{1}{2} \xi\right) h(\xi, \lambda)}{\left(1+\left|\lambda-\left(\xi^{3}-\xi\right)\right|\right)^{b_{1}}} \frac{\left|\xi_{1}\right|^{\frac{1}{4}} f\left(\xi_{1}, \lambda_{1}\right) g\left(\xi-\xi_{1}, \lambda-\lambda_{1}\right)}{\left(1+\left|\lambda_{1}-\left(\xi_{1}^{3}-\xi_{1}\right)\right|\right)^{b}} d \mu \\
& \\
& \quad:=B_{1}+B_{2} .
\end{aligned}
$$

Arguing as in case (b) yields

$$
B_{1} \leq C\|h\|_{L_{\xi}^{2} L_{\lambda}^{2}}\|g\|_{L_{\xi}^{2} L_{\tau}^{2}}\left\|D_{x}^{\frac{1}{8}} F_{b}\right\|_{L_{x}^{4} L_{t}^{4}} \text { and } B_{2} \leq C\|h\|_{L_{\xi}^{2} L_{\lambda}^{2}}\|f\|_{L_{\xi}^{2} L_{\tau}^{2}}\left\|D_{x}^{\frac{1}{4}} G_{b}\right\|_{L_{x}^{4} L_{t}^{3}} \text {. }
$$

Next consider the case $|\xi| \geq 2$. First assume $|\xi| \geq 2$ and $\left|\xi_{1}\right| \leq 1$ so that $\left|\xi-\xi_{1}\right| \geq 1$. In this domain,

$$
\frac{1}{2}|\xi| \leq\left|\xi-\xi_{1}\right| \leq 2|\xi|
$$


and so the integral $\Upsilon$ is bounded as follows:

$$
\begin{gathered}
\int_{R^{4}} \frac{|\xi|^{1-s} h(\xi, \lambda)}{\frac{\left|\xi-\xi_{1}\right|^{s} g\left(\xi-\xi_{1}, \lambda-\lambda_{1}\right)}{\left(1+\left|\lambda-\left(\xi^{3}-\xi\right)\right|\right)^{b_{1}}} \frac{\chi\left(\frac{1}{2} \xi\right) f\left(\xi_{1}, \lambda_{1}\right)}{\left(1+\left|\lambda_{1}\right|\right)^{\alpha}}} \\
=\left\langle D_{x}^{1-s} H_{b_{1}}, \mathcal{F}_{\alpha} D_{x}^{s} G_{b}\right\rangle \\
\leq C\left\|D_{x}^{1-s} H_{b_{1}}\right\|_{L_{x}^{\frac{2}{s}} L_{t}^{2}}\left\|\mathcal{F}_{\alpha}\right\|_{L_{x}^{2} L_{t}^{\infty}}\left\|D_{x}^{s} G_{b}\right\|_{L_{x}^{1-s}} L_{t}^{2} \\
\leq C\|h\|_{L_{\xi}^{2} L_{\tau}^{2}}\left\|\mathcal{F}_{\alpha}\right\|_{L_{x}^{2} L_{t}^{\infty}}\left\|D_{x}^{s} G_{b}\right\|_{L_{x}^{1-s}} L_{t}^{2} \\
\leq C\|h\|_{L_{\xi}^{2} L_{\tau}^{2}}\left\|\mathcal{F}_{\alpha}\right\|_{L_{x}^{2} L_{t}^{\infty}}\left\|D_{x}^{s} G_{b}\right\|_{L_{x}^{\frac{2}{1-s}} L_{t}^{2}}
\end{gathered}
$$

where $\widehat{\mathcal{F}}_{\alpha}(\xi, \lambda)=\frac{\chi\left(\frac{1}{2} \xi\right) f(\xi, \lambda)}{(1+|\lambda|)^{\alpha}}$. In these machinations, it was required that

$$
0 \leq s \leq 1, b_{1}>\frac{1}{2}(1-s), b>\frac{s}{2}, \alpha>\frac{1}{2} .
$$

Assume now that $|\xi| \geq 2,\left|\xi_{1}\right| \geq 1$ and $\left|\xi-\xi_{1}\right| \geq\left|\xi_{1}\right|$. In this region, the inequality $|\xi| \leq 2\left|\xi-\xi_{1}\right|$ holds and therefore the integral $\Upsilon$ is bounded above by

$$
\begin{aligned}
& \int_{R^{4}} \frac{|\xi|^{1-s} h(\xi, \lambda)}{\left(1+\left|\lambda-\left(\xi^{3}-\xi\right)\right|\right)^{b_{1}}} \\
& \frac{\left|\xi_{1}\right|^{s} f\left(\xi_{1}, \lambda_{1}\right)\left|\xi-\xi_{1}\right|^{s} g\left(\xi-\xi_{1}, \lambda-\lambda_{1}\right)}{\left(1+\left|\lambda_{1}-\left(\xi_{1}^{3}-\xi_{1}\right)\right|\right)^{b}\left(1+\left|\lambda-\lambda_{1}-\left(\left(\xi-\xi_{1}\right)^{3}-\left(\xi-\xi_{1}\right)\right)\right|\right)^{b}} d \mu .
\end{aligned}
$$

The domain of integration of the latter integral is again considered in the three subcases as in (5.11). If (a) holds,

$$
\begin{aligned}
& \int_{R^{4}} \frac{|\xi|^{1-s} h(\xi, \lambda)}{\left(1+\left|\lambda-\left(\xi^{3}-\xi\right)\right|\right)^{b_{1}}} \\
& \frac{\left|\xi_{1}\right|^{s} f\left(\xi_{1}, \lambda_{1}\right)\left|\xi-\xi_{1}\right|^{s} g\left(\xi-\xi_{1}, \lambda-\lambda_{1}\right)}{\left(1+\left|\lambda_{1}-\left(\xi_{1}^{3}-\xi_{1}\right)\right|\right)^{b}\left(1+\left|\lambda-\lambda_{1}-\left(\left(\xi-\xi_{1}\right)^{3}-\left(\xi-\xi_{1}\right)\right)\right|\right)^{b}} d \mu \\
& \leq C \int_{R^{4}}|\xi|^{1-s-b_{1}} h(\xi, \lambda) \\
& \frac{\left|\xi_{1}\right|^{s-b_{1}} f\left(\xi_{1}, \lambda_{1}\right)\left|\xi-\xi_{1}\right|^{s-b_{1}} g\left(\xi-\xi_{1}, \lambda-\lambda_{1}\right)}{\left(1+\left|\lambda_{1}-\left(\xi_{1}^{3}-\xi_{1}\right)\right|\right)^{b}\left(1+\left|\lambda-\lambda_{1}-\left(\left(\xi-\xi_{1}\right)^{3}-\left(\xi-\xi_{1}\right)\right)\right|\right)^{b}} d \mu \\
& \leq C \int_{R^{4}} h(\xi, \lambda) \frac{\left.\left|\xi_{1}\right|^{\frac{1}{8}} f\left(\xi_{1}, \lambda_{1}\right)\left|\xi-\xi_{1}\right|^{\frac{1}{8}} g\left(\xi-\xi_{1}, \lambda-\lambda\right)_{1}\right)}{\left(1+\left|\lambda_{1}-\left(\xi_{1}^{3}-\xi_{1}\right)\right|\right)^{b}\left(1+\left|\lambda-\lambda_{1}-\left(\left(\xi-\xi_{1}\right)^{3}-\left(\xi-\xi_{1}\right)\right)\right|\right)^{b}} d \mu \\
& \leq C\|h\|_{L_{\xi}^{2} L_{\lambda}^{2}}\left\|D_{x}^{\frac{1}{8}} F_{b}\right\|_{L_{x}^{4} L_{t}^{4}}\left\|D_{x}^{\frac{1}{8}} G_{b}\right\|_{L_{x}^{4} L_{t}^{4}} \\
& \leq C\|h\|_{L_{\xi}^{2} L_{\lambda}^{2}}\|g\|_{L_{\xi}^{2} L_{\lambda}^{2}}\left\|D_{x}^{\frac{1}{8}} F_{b}\right\|_{L_{x}^{4} L_{t}^{4}}
\end{aligned}
$$


where it was required that

$$
b_{1}-s+\frac{1}{8} \geq 0, \quad b_{1} \geq \frac{7}{16}, \quad s-b_{1} \leq \frac{1}{8} .
$$

If (b) holds, $\Upsilon$ is bounded above by

$$
\begin{aligned}
& \int_{R^{4}} \frac{|\xi|^{1-s} h(\xi, \lambda)}{\left(1+\left|\lambda-\left(\xi^{3}-\xi\right)\right|\right)^{b_{1}}} \\
& \frac{\left|\xi_{1}\right|^{s} f\left(\xi_{1}, \lambda_{1}\right)\left|\xi-\xi_{1}\right|^{s} g\left(\xi-\xi_{1}, \lambda-\lambda_{1}\right)}{\left(1+\left|\lambda_{1}-\left(\xi_{1}^{3}-\xi_{1}\right)\right|\right)^{b}\left(1+\left|\lambda-\lambda_{1}-\left(\left(\xi-\xi_{1}\right)^{3}-\left(\xi-\xi_{1}\right)\right)\right|\right)^{b}} d \mu \\
& \leq C \int_{R^{4}}|\xi|^{1-s-b} h(\xi, \lambda)\left|\xi_{1}\right|^{s-b} f\left(\xi_{1}, \lambda_{1}\right) \frac{\left|\xi-\xi_{1}\right|^{s-b} g\left(\xi-\xi_{1}, \lambda-\lambda_{1}\right)}{\left(1+\left|\lambda-\lambda_{1}-\left(\left(\xi-\xi_{1}\right)^{3}-\left(\xi-\xi_{1}\right)\right)\right|\right)^{b}} d \mu \\
& \leq C \int_{R^{4}} h(\xi, \lambda) f\left(\xi_{1}, \lambda_{1}\right) \frac{\left|\xi-\xi_{1}\right|^{1 / 4} g\left(\xi-\xi_{1}, \lambda-\lambda_{1}\right)}{\left(1+\left|\lambda-\lambda_{1}-\left(\left(\xi-\xi_{1}\right)^{3}-\left(\xi-\xi_{1}\right)\right)\right|\right)^{b}} d \mu \\
& \leq C\|h\|_{L_{\xi}^{2} L_{\lambda}^{2}}\|f\|_{L_{\xi}^{2} L_{\tau}^{2}}\left\|D_{x}^{\frac{1}{4}} G_{b}\right\|_{L_{x}^{4} L_{t}^{3}},
\end{aligned}
$$

the validation of which subsists on the conditions

$$
b_{1} \geq \frac{7}{16}, \quad 3 b-s>\frac{3}{4}, \quad s-b \leq \frac{1}{8} .
$$

If (c) holds, we bound $\Upsilon$ above as follows:

$$
\begin{aligned}
& \int_{R^{4}} \frac{|\xi|^{1-s} h(\xi, \lambda)}{\left(1+\left|\lambda-\left(\xi^{3}-\xi\right)\right|\right)^{b_{1}}} \\
& \frac{\left|\xi_{1}\right|^{s} f\left(\xi_{1}, \lambda_{1}\right)\left|\xi-\xi_{1}\right|^{s} g\left(\xi-\xi_{1}, \lambda-\lambda_{1}\right)}{\left(1+\left|\lambda_{1}-\left(\xi_{1}^{3}-\xi_{1}\right)\right|\right)^{b}\left(1+\left|\lambda-\lambda_{1}-\left(\left(\xi-\xi_{1}\right)^{3}-\left(\xi-\xi_{1}\right)\right)\right|\right)^{b}} d \mu \\
& \leq C \int_{R^{4}} \frac{|\xi|^{1-s-b} h(\xi, \lambda)}{\left(1+\left|\lambda-\left(\xi^{3}-\xi\right)\right|\right)^{b_{1}}} \frac{\left|\xi_{1}\right|^{2(s-b)} f\left(\xi_{1}, \lambda_{1}\right) g\left(\xi-\xi_{1}, \lambda-\lambda_{1}\right)}{\left(1+\left|\lambda_{1}-\left(\xi_{1}^{3}-\xi_{1}\right)\right|\right)^{b}} d \mu+ \\
& \quad+\int_{R^{4}} \frac{|\xi|^{1-2 b} h(\xi, \lambda)}{\left(1+\left|\lambda-\left(\xi^{3}-\xi\right)\right|\right)^{b_{1}}} \frac{\left|\xi_{1}\right|^{s-b} f\left(\xi_{1}, \lambda_{1}\right) g\left(\xi-\xi_{1}, \lambda-\lambda_{1}\right)}{\left(1+\left|\lambda_{1}-\left(\xi_{1}^{3}-\xi_{1}\right)\right|\right)^{b}} d \mu \\
& \leq C\|f\|_{L_{\xi}^{2} L_{\tau}^{2}}\|g\|_{L_{\xi}^{2} L_{\tau}^{2}\left\|D_{x}^{1 / 8} F_{b}\right\|_{L_{x}^{4} L_{t}^{4}},}
\end{aligned}
$$

for which we needed

$$
s-b \leq \frac{1}{16}, \quad b \geq \frac{7}{16} .
$$

Let $\alpha_{0}=\max \left\{\frac{s}{2}, \frac{7}{16}\right\}, \alpha_{0}<b<\frac{1}{2}$ and $\gamma_{0}=\frac{1}{2}-b$. Then, using the same argument as that appearing in the proof of Lemma 3.4 in [33], one sees that the quantities

are bounded by

$$
\left\|D_{x}^{\frac{1}{4}} F_{b}\right\|_{L_{x}^{4} L_{t}^{3}},\left\|D_{x}^{\frac{1}{8}} F_{b}\right\|_{L_{x}^{4} L_{t}^{4}},\left\|F_{b}\right\|_{L_{x}^{4} L_{t}^{4}}
$$

$$
C \delta^{\frac{\gamma_{0}}{4 b}}\|p\|_{X_{-s, b}^{\alpha}}
$$

and for $0 \leq s^{\prime} \leq 1$, the quantities

$$
\left\|D_{x}^{\frac{1}{4}} G_{b}\right\|_{L_{x}^{4} L_{t}^{3}},\left\|D_{x}^{s^{\prime}} G_{b}\right\|_{L_{x}^{\frac{2}{1-s^{\prime}}} L_{t}^{2}}
$$


are bounded by

$$
C \delta^{\frac{\gamma_{0}}{4 b}}\|q\|_{X_{-s, b}^{\alpha}}
$$

where $C$ is a constant independent of $\delta, p$ and $q$. Consequently, by Lemma 4.1, it must be the case that

$$
\begin{gathered}
\mathcal{P}_{\alpha}(w)+\mathcal{Q}_{-s, b_{1}}(w) \leq C \delta^{\frac{\gamma_{0}}{4 b}}\|p\|_{X_{-s, b}^{\alpha}}\|q\|_{X_{-s, b}^{\alpha}} \\
\leq C \delta^{1-2 \alpha+\frac{\gamma_{0}}{4 b}}\|p\|_{X_{-s, b}^{\alpha}}\|q\|_{X_{-s, b}^{\alpha}} .
\end{gathered}
$$

One may choose $\alpha>\frac{1}{2}$ such that

$$
\theta_{0}=1-2 \alpha+\frac{\gamma_{0}}{4 b}>0 .
$$

to obtain the estimates (5.8) and (5.9).

Now, notice that

$$
\begin{aligned}
& \int_{R}\left(\int_{R} \frac{|\hat{u}(\xi, \lambda)|}{1+\left|\lambda-\left(\xi^{3}-\xi\right)\right|} d \lambda\right)^{2} d \xi \\
= & \int_{R}\left(\int_{R} \frac{|\hat{u}(\xi, \lambda)|}{\left(1+\left|\lambda-\left(\xi^{3}-\xi\right)\right|\right)^{b_{1}}} \frac{1}{\left(1+\left|\lambda-\left(\xi^{3}-\xi\right)\right|\right)^{1-b_{1}}} d \lambda\right)^{2} d \xi \\
\leq & \int_{R}\left(\int_{R} \frac{|\hat{u}(\xi, \lambda)|^{2}}{\left(1+\left|\lambda-\left(\xi^{3}-\xi\right)\right|\right)^{2 b_{1}}} d \lambda\right)\left(\int_{R} \frac{1}{\left(1+\left|\lambda-\left(\xi^{3}-\xi\right)\right|\right)^{2\left(1-b_{1}\right)}} d \lambda\right) d \xi .
\end{aligned}
$$

Since $b_{1}<\frac{1}{2}$ and $2\left(1-b_{1}\right)>1$, the second $\lambda$-integral is bounded and only the estimate of the first term is needed. Thus (5.10) follows from (5.8) and (5.9). The proof is complete.

5.2. Appendix II. Observe that, if $b \geq 0, \tau \in R$ and $-1 \leq \xi \leq 1$, then there are constants $C_{1}$ and $C_{2}$ such that

$$
C_{1}(1+|\tau|)^{2 b} \leq\left(1+\left|\tau-\left(\xi^{3}-\xi\right)\right|^{2 b} \leq C_{2}(1+|\tau|)^{2 b}\right.
$$

and

Thus, if $s \in R$ and $b \geq 0$,

$$
C_{1} \leq(1+|\xi|)^{s} \leq C_{2}
$$

$$
\lambda_{b}(w) \leq C \Lambda_{s, b}(w) .
$$

The following estimate then follows directly from Theorem 3.7.

LEMMA 5.1. Let $\psi(t)$ be a smooth function of $t$ with compact support. For any $\alpha \geq 0$, there exists a constant $C$ such that

$$
\lambda_{\alpha}\left(\psi \mathcal{B} I_{e} h\right) \leq C\|h\|_{H^{\frac{3 \alpha-1}{3}}}\left(R^{+}\right)
$$

for any $h \in H_{0}^{\frac{3 \alpha-1}{3}}\left(R^{+}\right)$.

The next lemma shows that the estimate (5.12) is optimal as regards the Sobolev index on its right-hand side.

LEMMA 5.2. The estimate (5.12) in Lemma 5.1 fails if $H_{0}^{\frac{3 \alpha-1}{3}}\left(R^{+}\right)$is replaced by $H_{0}^{\frac{3 \alpha-1}{3}-\epsilon}\left(R^{+}\right)$for any $\epsilon>0$. 
Proof: Return to the proof of the inequalities in Theorem 3.7 and use the notation in force there. All we need to consider is the estimate of $I_{2}(x, t)$ since it has been demonstrated in the proof of Theorem 3.1 that

$$
\Lambda_{s, b}\left(\psi I_{1}\right) \leq C\|h\|_{L^{2}\left(R^{+}\right)}
$$

for any $b \geq 0$ and $s \in R$, where the constant $C$ depends only on $\psi, b$ and $s$. Recall that

$$
\begin{gathered}
\mathcal{F}_{x}\left[\operatorname{ReI}_{2}(x, t)\right]= \\
\int_{\frac{2}{\sqrt{3}}}^{\infty} \int_{0}^{\infty}\left(\int_{0}^{\infty} \cos (x \xi) \cos \left(\lambda(t-s)-\frac{\mu(\lambda)}{2} x\right) e^{-x \frac{\sqrt{3 \mu^{2}(\lambda)-4}}{2}} d x\right) \phi_{1}(\mu(\lambda)) h(s) d s d \lambda .
\end{gathered}
$$

Now suppose $|\xi| \leq 1$ and study the behavior of the kernel as $\lambda \rightarrow+\infty$. Because of an earlier computation, it is known that

$$
\begin{gathered}
\quad \int_{0}^{\infty} \cos (x \xi) \cos \left(\lambda(t-s)-\frac{\mu(\lambda)}{2} x\right) e^{-x \frac{\sqrt{\mu^{2}(\lambda)-4}}{2}} d x \\
=-\frac{\sqrt{3}}{2 \lambda^{\frac{1}{3}}}(\cos (\lambda(t+s))-\sin (\lambda(t+s))) \\
\quad+A_{0}(\xi, \lambda) \cos (\lambda(t-s))+B_{0}(\xi, \lambda) \sin (\lambda(t-s)) \\
=-\frac{1}{\lambda^{1 / 3}} \cos \left(\lambda(t-s)+\frac{1}{6} \pi\right)+A_{0}(\xi, \lambda) \cos (\lambda(t-s))+B_{0}(\xi, \lambda) \sin (\lambda(t-s))
\end{gathered}
$$

where

$$
\left|A_{0}(\xi, \lambda)\right|+\left|B_{0}(\xi, \lambda)\right| \leq \frac{C}{\lambda^{\frac{2}{3}}}
$$

uniformly for $|\xi| \leq 1$. The estimates for $A_{0}(\xi, \lambda) \cos (\lambda(t-s))$ and $B_{0}(\xi, \lambda) \sin (\lambda(t-$ $s)$ ) can be obtained as before and the results are better in fact. Thus, attention may be focused on the term corresponding to the portion

of the kernel, namely

$$
-\frac{1}{\lambda^{\frac{1}{3}}} \cos \left(\lambda(t-s)+\frac{1}{6} \pi\right),
$$

$$
\begin{aligned}
I I_{1}(t) & =\int_{\frac{2}{\sqrt{3}}}^{\infty} \int_{0}^{\infty} \frac{1}{\lambda^{\frac{1}{3}}} \cos \left(\lambda(t-s)+\frac{1}{6} \pi\right) \phi_{1}(\mu(\lambda)) h(s) d s d \lambda \\
:= & B_{1}(t)+\overline{B_{1}(t)}
\end{aligned}
$$

with

$$
B_{1}(t)=\frac{1}{2} \int_{\frac{2}{\sqrt{3}}}^{\infty} e^{i \lambda t} \frac{\phi_{1}(\mu(\lambda))}{\lambda^{\frac{1}{3}}} \int_{0}^{\infty} e^{-i\left(\lambda s-\frac{1}{6} \pi\right)} h(s) d s d \lambda .
$$

Taking the Fourier transform in the temporal variable leads to

$$
\begin{aligned}
\mathcal{F}_{t}\left[I I_{1}(t)\right](\tau) & =\frac{1}{2} \frac{\phi_{1}(\mu(\tau))}{\tau^{\frac{1}{3}}} \int_{0}^{\infty} e^{-i\left(\lambda s-\frac{1}{6} \pi\right)} h(s) d s \\
& +\frac{1}{2} \frac{\phi_{1}(\mu(-\tau))}{-\tau^{\frac{1}{3}}} \int_{0}^{\infty} e^{-\left(\lambda s+\frac{1}{6} \pi\right)} h(s) d s .
\end{aligned}
$$


For any smooth function $\psi(t)$ with compact support in $R$, we have

$$
\begin{gathered}
\mathcal{F}_{t}\left[\psi(t) I I_{1}(t)\right](\tau)=\mathcal{F}_{t}[\psi(t)](\tau) * \mathcal{F}_{t}\left[I I_{1}(t)\right](\tau) \\
=\int_{\frac{2}{\sqrt{3}}}^{\infty} \hat{\psi}\left(s_{1}\right) \mathcal{F}_{t}\left[I I_{1}(t)\right]\left(\tau-s_{1}\right) d s_{1}
\end{gathered}
$$

Of course, for any $n>0, \hat{\psi}(\tau) \sim|\tau|^{-n}$ as $\tau \rightarrow \infty$. Break the last integral into two parts, viz.

$$
\mathcal{F}_{t}\left[\psi(t) I I_{1}(t)\right](\tau)=\left(\int_{\left|s_{1}\right|>\sqrt{|\tau|}}+\int_{\left|s_{1}\right| \leq \sqrt{|\tau|}}\right) \hat{\psi}\left(s_{1}\right) \mathcal{F}_{t}\left[I I_{1}(t)\right]\left(\tau-s_{1}\right) d s_{1}
$$

The term

$$
\int_{\left|s_{1}\right|>\sqrt{|\tau|}} \hat{\psi}\left(s_{1}\right) \mathcal{F}_{t}\left[I I_{1}(t)\right]\left(\tau-s_{1}\right) d s_{1}
$$

decays rapidly as $|\tau| \rightarrow \infty$. Consequently, we look carefully at the term

$$
\begin{aligned}
& I I_{3}(\tau)=\int_{\left|s_{1}\right| \leq \sqrt{|\tau|}} \hat{\psi}\left(s_{1}\right)\left[I I_{1}(t)\right]\left(\tau-s_{1}\right) d s_{1} \\
& =\int_{\left|s_{1}\right| \leq \sqrt{|\tau|}} \frac{\hat{\psi}\left(s_{1}\right)}{2\left(\tau-s_{1}\right)^{1 / 3}} m\left(\mu\left(\tau-s_{1}\right)\right) \int_{0}^{\infty} e^{-i\left(\tau-s_{1}\right)} h(s) d s d s_{1} \\
& =\frac{1}{2 \tau^{\frac{1}{3}}} \int_{\left|s_{1}\right| \leq \sqrt{|\tau|}} \hat{\psi}\left(s_{1}\right) m\left(\mu\left(\tau-s_{1}\right)\right) \int_{0}^{\infty} e^{-i\left(\tau-s_{1}\right)} h(s) d s d s_{1}+O\left(\tau^{-\frac{2}{3}}\right)
\end{aligned}
$$

where

$$
m\left(\mu\left(\tau-s_{1}\right)\right)=\phi_{1}\left(\mu\left(\tau-s_{1}\right)\right) e^{i \frac{\pi}{6}}-\phi_{1}\left(\mu\left(s_{1}-\tau\right)\right) e^{-i \frac{\pi}{6}}
$$

As $\tau \rightarrow+\infty$,

$$
\begin{aligned}
& I I_{3}(\tau)=\frac{1}{2 \tau^{\frac{1}{3}}} \int_{\left|s_{1}\right| \leq \sqrt{|\tau|}} \hat{\psi}\left(s_{1}\right) e^{i \frac{\pi}{6}} \int_{0}^{\infty} e^{-i\left(\tau-s_{1}\right) s} h(s) d s d s_{1}+O\left(\tau^{-\frac{2}{3}}\right) \\
& =\frac{1}{4 \tau^{\frac{1}{3}}} \int_{1}^{\infty} \hat{\psi}\left(s_{1}\right) \int_{1}^{\infty} e^{-i\left(\tau-s_{1}\right) s} e^{i \frac{\pi}{6}} \tilde{h}(s) d s d s_{1}+O\left(\tau^{-2 / 3}\right) \\
& =\frac{1}{4 \tau^{\frac{1}{3}}} \mathcal{F}_{s}\left[\psi(s) e^{i \frac{\pi}{6}} \tilde{h}(s)\right](\tau)+O\left(\tau^{-\frac{2}{3}}\right)
\end{aligned}
$$


where $\tilde{h}(s)$ is the even extension of $h(s)$. Estimate the semi-norm $\lambda_{\alpha}$ of $\psi(t) I_{2}(x, t)$ as before:

$$
\begin{aligned}
& \lambda_{\alpha}\left(\psi I_{2}\right) \geq C \int_{1}^{\infty} \int_{0}^{1}|\tau|^{\alpha}\left|\mathcal{F}_{t} \mathcal{F}_{x}\left[I I_{1}(x, t) \psi(t)\right]\right|^{2} d \xi d \tau \\
& \geq C \int_{0}^{\infty} \int_{0}^{1}|\tau|^{\alpha}\left(\left|\frac{1}{\tau^{\frac{1}{3}}} \mathcal{F}_{s}\left[\psi(s) e^{i \frac{\pi}{6}} \tilde{h}(s)\right](\tau)\right|^{2}\right. \\
& \left.\quad-\left|\frac{1}{\tau^{\frac{2}{3}}} \phi_{1}^{2}(\mu(\tau))\left(\int_{0}^{\infty} h(s) e^{-i s \tau}\right)^{2}\right|\right) d \tau \\
& \geq C\left(\int_{0}^{\infty} \tau^{2 \alpha-\frac{2}{3}}\left|\mathcal{F}_{s}\left[\psi(s) e^{i \frac{\pi}{6}} \tilde{h}(s)\right](\tau)\right|^{2} d \tau-\int_{0}^{\infty} \tau^{2 \alpha-\frac{4}{3}}\left|\int_{0}^{\infty} h(s) e^{-i s \tau} d s\right|^{2} d \tau\right) \\
& \geq C\left(\|\psi(t) \tilde{h}(t)\|_{H_{t}^{\alpha-\frac{1}{3}}\left(R^{+}\right)}^{2}-\|h\|_{H^{\alpha-\frac{2}{3}}\left(R^{+}\right)}^{2}\right) \cdot
\end{aligned}
$$

Thus, if we choose $h(t)=h_{n}(t)$ such that

(i) $\left\|\psi(t) h_{n}(t)\right\|_{H^{\alpha-1 / 3}} \rightarrow+\infty$ as $n \rightarrow \infty$,

(ii) $\left\|h_{n}\right\|_{H^{\alpha-2 / 3}}$ bounded independently of $n$ and

(iii) $h_{n}$ converges to $h^{*}$ in the space $H_{0}^{\frac{3 \alpha-1}{3}-\epsilon}\left(R^{+}\right)$),

then it follows that

$$
\lambda_{\alpha}\left(I I_{2, n}(x, t) \psi(t)\right) \rightarrow \infty
$$

as $n \rightarrow \infty$. So, inequality (3.17) cannot hold with $H_{0}^{\frac{3 \alpha-1}{3}}\left(R^{+}\right)$replaced by $H_{0}^{\frac{3 \alpha-1}{3}-\epsilon}\left(R^{+}\right)$, no matter how small is $\epsilon>0$. The proof is complete

\section{References}

[1] J. L. Bona and P. J. Bryant, A mathematical model for long waves generated by wave makers in nonlinear dispersive systems, Proc. Cambridge Philos. Soc. 73 (1973), $391-405$.

[2] J. L. Bona and L. Luo, Generalized Korteweg-de Vries equation in a quarter plane, Contemp. Math. 221 (1999), $59-125$.

[3] J. L. Bona and L. R. Scott, Solutions of the Korteweg-de Vries equation in fractional order Sobolev spaces, Duke Math. J. 43 (1976), $87-99$.

[4] J. L. Bona, S. Sun and B.-Y. Zhang, A nonhomogeneous boundary-value problem for the Korteweg-de Vries equation in a quarter plane, Trans. American Math. Soc. 354 (2001), 427 -490 .

[5] J. L. Bona, S. Sun and B.-Y. Zhang, Forced oscillations of a damped KdV equation in a quarter plane, Commun. Contemp. Math. 5 (2003), 369-400.

[6] J. L. Bona, S. Sun and B.-Y. Zhang, A nonhomogeneous boundary value problem for the KdV equation posed on a finite domain, Commun. Partial Differential Eq. 28 (2003), 1391-1436.

[7] J. L. Bona, S. Sun and B.-Y. Zhang, Conditional and unconditional well-posedness of nonlinear evolution equations, Adv. Differential Eq., 9 (2004), $241-265$.

[8] J. L. Bona and R. Winther, The Korteweg-de Vries equation, posed in a quarter plane, SIAM J. Math. Anal. 14 (1983), 1056 - 1106.

[9] J. L. Bona and R. Winther, Korteweg-de Vries equation in a quarter plane, continuous dependence results, Differential Integral Eq. 2 (1989), 228 - 250.

[10] J. Bourgain, Fourier transform restriction phenomena for certain lattice subsets and applications to non-linear evolution equations, part I: Schrödinger equations, Geom. \&s Funct. Anal. 3 (1993), 107 - 156. 
[11] J. Bourgain, Fourier transform restriction phenomena for certain lattice subsets and applications to non-linear evolution equations, part II: the KdV equation, Geom. \&3 Funct. Anal. 3 (1993), $209-262$.

[12] J. E. Colliander and C. E. Kenig, The generalized Korteweg-de Vries equation on the half line, Comm. Partial Differential Eq., 27 (2002), 2187-2266.

[13] J. E. Colliander, M. Keel, G. Staffilani, H. Takaoka and T. Tao, Sharp global well-posedness for KdV and modified KdV on $R$ and T, J. American Math. Soc. 16 (2003), 705-749

[14] P. Constantin and J.-C. Saut, Local smoothing properties of dispersive equations, J. American Math. Soc. 1 (1988), $413-446$.

[15] W. Craig, T. Kappeler and W. A. Strauss, Gain of regularity for equations of the Korteweg-de Vries type, Ann. Inst. Henri Poincaré 9 (1992), 147 - 186.

[16] A. V. Faminskii, The Cauchy problem and the mixed problem in the half strip for equations of Korteweg- de Vries type, (Russian) Dinamika Sploshn. Sredy 162 (1983), 152 - 158.

[17] A. V. Faminskii, A mixed problem in a semistrip for the Korteweg-de Vries equation and its generalizations, (Russian) Dinamika Sploshn. Sredy 258 (1988), 54 - 94; English transl. in Trans. Moscow Math. Soc. 51 (1988).

[18] A. V. Faminskii, Mixed problems for the Korteweg-de Vries equation, Sbornik: Mathematics 190 (1999), 903-935.

[19] A. V. Faminskii, An initial boundary-value problem in a half-strip for the Korteweg-de Vries equation in fractional-order Sobolev spaces, Comm. Partial Differential Eq. 29 (2004), 16531695 ,

[20] A. S. Fokas and B. Pelloni, The solution of certain initial boundary-value problems for the linearized Korteweg-de Vries equation, Proc. Royal. Soc. London Series A 454 (1998), 645 -657 .

[21] A. S. Fokas and A. R. Its, Integrable equations on the half-infinite line. Solitons in science and engineering: theory and applications. Chaos Solitons Fractals 5 (1995), $2367-2376$.

[22] A. S. Fokas and A. R. Its, An initial-boundary value problem for the Korteweg-de Vries equation, In the proceedings of the conference: Solitons, nonlinear wave equations and computation (New Brunswick, NJ, 1992), Math. Comput. Simulation 37 (1994), 293 - 321.

[23] A. S. Fokas and A. R. Its, Soliton generation for initial-boundary value problems, Phys. Rev. Lett. 68 (1992), $3117-3120$

[24] G. H. Hardy, J. E. Littlewood and G. Pólya, Inequalities, Cambridge University Press: Cambridge (1934).

[25] J. Holmer, The initial-boundary value problem for the Korteweg-de Vries equation, preprint (2005).

[26] T. Kato, Quasilinear equations of evolution with applications to partial differential equations, Springer Lecture Notes in Math. 448 (1975), 27 - 50.

[27] T. Kato, On the Korteweg-de Vries equation, Manuscripta Math. 29 (1979), 89 - 99.

[28] T. Kato, The Cauchy problem for the Korteweg-de Vries equation, Pitman Research Notes in Math. 53 (1979), 293 - 307.

[29] T. Kato, On the Cauchy problem for the (generalized) Korteweg-de Vries equations, Advances in Mathematics Supplementary Studies, Studies Appl. Math. 8 (1983), 93 - 128.

[30] T. Kato, On nonlinear Scrhödinger equations. II. $H^{s}-$ solutions and unconditional wellposedness, J. d'Anal. Math. 67 (1995), 281-306.

[31] C. E. Kenig, G. Ponce and L. Vega, Well-posedness of the initial value problem for the KdV equation, J. American Math. Soc. 4 (1991), $323-347$.

[32] C. E. Kenig, G. Ponce and L. Vega, Well-posedness and scattering results for the generalized Korteweg-de Vries equations via the contraction principle, Comm. Pure Appl. Math. 46 (1993), $527-620$.

[33] C. E. Kenig, G. Ponce and L. Vega, The Cauchy problem for the Korteweg-de Vries equation in Sobolev spaces of negative indices, Duke Math. J. 71 (1993), $1-21$.

[34] C. E. Kenig, G. Ponce and L. Vega, A bilinear estimate with applications to the KdV equation, J. American Math. Soc. 9 (1996), $573-603$.

[35] J.-L. Lions and E. Magenes, Non-homogeneous boundary value problems and applications, Vol. 1, Springer-Verlag: Heidelberg (1972).

[36] L. Molinet and F. Ribaud, On the low regularity of the Korteweg-de Vries-Burgers equation, International Math. Res. Notices, 37 (2002), 1979-2005. 
[37] D. L. Russell and B.-Y. Zhang, Smoothing properties of solutions of the Korteweg-de Vries equation on a periodic domain with point dissipation, J. Math. Anal. Appl., 190 (1995), 449 $-488$.

[38] J.-C. Saut and R. Temam, Remarks on the Korteweg-de Vries equation, Israel J. Math. 24 (1976), $78-87$

[39] P. Sjölin, Regularity of solutions to the Schödinger equation, Duke Math. J. 55 (1987), 699 715 .

[40] E. M. Stein and G. Weiss, Introduction to Fourier Analysis on Euclidean Spaces, Princeton University Press: Princeton (1971).

[41] L. Tartar, Interpolation non linèaire et régularité, J. Funct. Anal. 9 (1972), 469 - 489.

[42] B. A. Ton, Initial boundary value problems for the Korteweg-de Vries equation, J. Differential Eq. 25 (1977), 288-309.

[43] L. Vega, Schrödinger equations: pointwise convergence to the initial data, Proc. American Math. Soc. 102 (1988), $874-878$.

[44] B.-Y. Zhang, Taylor series expansion for solutions of the KdV equation with respect to their initial values, J. Funct. Anal. 129 (1995), 293 - 324.

[45] B.-Y. Zhang, Analyticity of solutions for the generalized Korteweg-de Vries equation with respect to their initial datum, SIAM J. Math. Anal. 26 (1995), 1488 - 1513.

[46] B.-Y. Zhang, A remark on the Cauchy problem for the Korteweg-de Vries equation on a periodic domain, Differential Integral Eq. 8 (1995), 1191 - 1204.

Department of Mathematics, Statistics \& Computer Science, University of Illinois at Chicago, Chicago, IL 60607

E-mail address: bona@math.uic.edu

Department of Mathematics, Virginia Polytechnic Institute and State University, Blacksburg, Virginia 24061-4097

E-mail address: sun@math.vt.edu

Department of Mathematical Sciences, University of Cincinnati, Cincinnati, Ohio 45221-0025

E-mail address: bzhang@math.uc.edu 